San Jose State University

SJSU ScholarWorks

Master's Theses

Master's Theses and Graduate Research

Summer 2018

\title{
Characterization of Four Type-C Silica Columns Using Resveratrol Analogues
}

Joshua Topete

San Jose State University

Follow this and additional works at: https://scholarworks.sjsu.edu/etd_theses

\section{Recommended Citation}

Topete, Joshua, "Characterization of Four Type-C Silica Columns Using Resveratrol Analogues" (2018). Master's Theses. 4956.

DOI: https://doi.org/10.31979/etd.33mq-3p59

https://scholarworks.sjsu.edu/etd_theses/4956

This Thesis is brought to you for free and open access by the Master's Theses and Graduate Research at SJSU ScholarWorks. It has been accepted for inclusion in Master's Theses by an authorized administrator of SJSU ScholarWorks. For more information, please contact scholarworks@sjsu.edu. 


\title{
CHARACTERIZATION OF FOUR TYPE-C SILICA COLUMNS USING RESVERATROL ANALOGUES
}

\author{
A Thesis \\ Presented to \\ The Faculty of the Department of Chemistry \\ San José State University \\ In Partial Fulfillment \\ of the Requirements for the Degree \\ Master of Science
}

by

Joshua Charles Topete

August 2018 
(C) 2018

Joshua Charles Topete

ALL RIGHTS RESERVED 
The Designated Thesis Committee Approves the Thesis Titled

CHARACTERIZATION OF FOUR TYPE-C SILICA COLUMNS USING RESVERATROL ANALOGUES

\author{
by \\ Joshua Charles Topete \\ APPROVED FOR THE DEPARTMENT OF CHEMISTRY \\ SAN JOSÉ STATE UNIVERSITY
}

August 2018

Joseph J. Pesek, Ph.D. D Department of Chemistry

Roger Terrill, Ph.D. Department of Chemistry

Chester Simocko, Ph.D. Department of Chemistry 


\section{ABSTRACT \\ CHARACTERIZATION OF FOUR TYPE-C SILICA COLUMNS USING RESVERATROL ANALOGUES}

\section{by Joshua Charles Topete}

High performance liquid chromatography (HPLC) is an analytical technique used to separate, identify and quantify components in a solution based on the affinities between analyte, mobile phase and stationary phase. The main goal of my research is to characterize four Type-C silica columns by comparing the retention of resveratrol analogues. The four columns included are functionalized with either Diamond Bidentate C18 (DBC18), Bidentate C18 (BC18), phenyl hydride or diol groups. Retention maps displaying mobile phase composition compared to compound retention times were compared between columns. In addition to this, two organic solvents (acetonitrile and methanol) were compared. Considering that the resveratrol analogues are fairly nonpolar, this set of compounds were expected to have slightly higher affinity for the DBC18 compared to the BC18. In comparison, the phenyl hydride column was anticipated to interact more with unsaturated compounds while the diol was likely to retain those with polar substituents. The data collected supported some of the expected trends based on polarity but each column demonstrated unique retention mechanisms for specific resveratrol analogues. The results determined that the BC18 column gave the highest retention strength while the phenyl hydride displayed the most selectivity for the resveratrol derivatives. 


\section{ACKNOWLEDGMENTS}

First and foremost, I would like to thank Drs. Maria Matyska-Pesek and Joseph Pesek for allowing me to work with and under them these many years. They have served as great mentors, role models and even parent-like figures in my life. From initial training, group meetings, presenting research and finally graduating this couple has assisted me and watched me grow the entire way. I also want to thank my other committee members Drs. Roger Terrill and Chester Simocko for being outstanding teachers and for agreeing to oversee my thesis work. I want to thank Michael Stephens, Akilu Kidane, Randy Kirchner and John Hawk for all the technical work and waste disposal taken care of behind the scenes. I want to give a special thanks to Sam Monroe and Stephen Cappelloni for all the service center assistance throughout the years. Working for the stockroom under Sam and Steve was my initial stepping stone into my chemistry career where I transitioned from being a complete delinquent to a somewhat diligent student. I also would like to thank the rest of the Chemistry Department and SAACS for all their help that tends to go unnoticed. Finally, I would like to thank God, my girlfriend, family and friends for all the moral support and advice throughout the years. My faith in God has kept me sane, my girlfriend has been extremely patient with me, my family and friends have always motivated and encouraged me and continue to do so. I am positive that everyone and anyone in my

life played or plays a role in my journey and I hope that I can do the same for you. Thank you all, I cannot thank you all enough. 


\section{TABLE OF CONTENTS}

List of Tables................................................................................................ viii

List of Figures.............................................................................................. ix

1. Introduction.............................................................................................

1.1 Background.................................................................................................

1.1.1 History of Chromatography.................................................................. 1

1.1.2 Types of Chromatography............................................................... 2

1.1.2.1 Homogeneous vs. heterogeneous................................................... 2

1.1.2.2 Adsorption vs. partition................................................................ 2

1.1.2.3 Columnar vs. planar....................................................................... 3

1.1.3 Main Chromatographic Modes................................................................ 4

1.1.3.1 Normal phase......................................................................... 4

1.1.3.2 Hydrophilic interaction................................................................ 4

1.1.3.3 Reversed phase.............................................................................. 5

1.1.3.4 Aqueous normal phase................................................................... 5

1.1.3.5 Other modes.............................................................................. 7

1.2 High Performance Liquid Chromatography................................................... 8

1.2.1 Applications in Modern HPLC........................................................... 9

1.2.2 Instrumental Components.................................................................. 10

1.2.2.1 Solvent manager....................................................................... 10

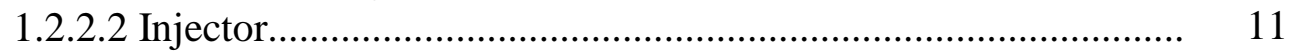

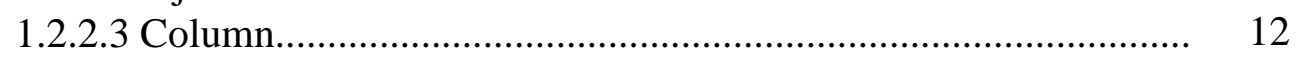

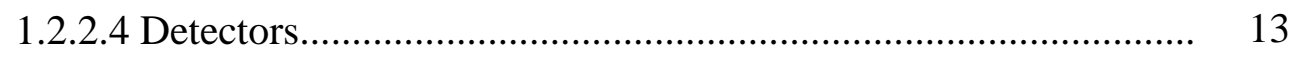

1.2.2.5 Computer and software.............................................................. 16

1.3 HPLC Parameters............................................................................... 16

1.3.1 Resolution................................................................................... 16

1.3.1.1 Retention Factor........................................................................ 17

1.3.1.2 Selectivity ........................................................................... 17

1.3.1.3 Efficiency............................................................................... 18

1.3.2 Van Deemter Equation.................................................................... 18

1.4 Stationary Phase - Silica......................................................................... 20

1.4.1 Advantages.................................................................................. 20

1.4.2 Evolution.............................................................................. 20

1.5 Synthesis........................................................................................... 22

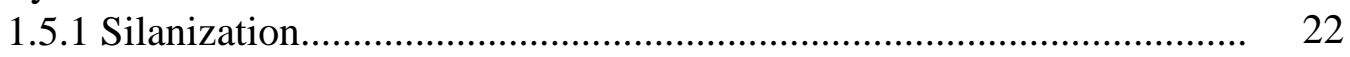

1.5.2 Hydrosilation................................................................................... 23

1.6 Research Aims and Goals...................................................................... 23 


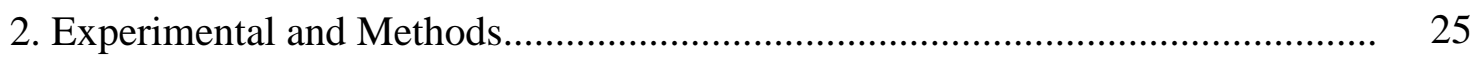

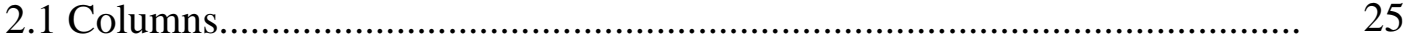

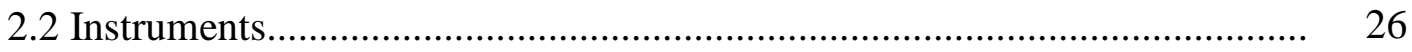

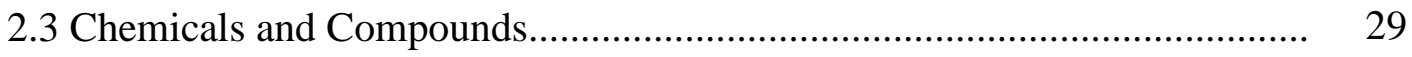

2.3.1 HPLC Solvents.......................................................................... 29

2.3.2 Resveratrol Analogues................................................................... 29

2.3.3 Acidic, Basic and Neutral Compounds......................................... 30

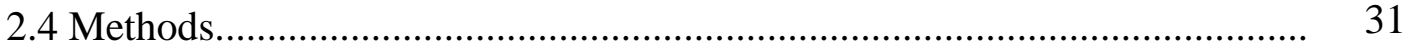

3. Results and Discussion.............................................................................. 33

3.1 Data with Acetonitrile as Mobile Phase.................................................. 36

3.1.1 Resveratrol Analogues................................................................ 36

3.1.2 Acidic, Basic and Neutral Compounds.......................................... 47

3.2 Data with Methanol as Mobile Phase......................................................... 57

3.2.1 Resveratrol Analogues............................................................... 57

3.2.2 Acidic, Basic and Neutral Compounds.......................................... 68

4. Conclusions and Future Studies..................................................................

5. References......................................................................................... 80 


\section{LIST OF TABLES}

Table 1. Column Specifications........................................................................ 26

Table 2. Wavelength Parameter.....................................................................

Table 3. Retention trends of resveratrol analogues based on structure and polarity................................................................................................. 35

Table 4. Diamond Bidentate C18 Capacity Factor " $k$ ” (ACN/Resveratrol) ........ 37

Table 5. Bidentate C18 Capacity Factor “ $k$ ” (ACN/Resveratrol) ......................... 41

Table 6. Phenyl Hydride Capacity Factor "k” (ACN/Resveratrol) ....................... 41

Table 7. Diol Capacity Factor " $k$ ” (ACN/Resveratrol) ......................................... 46

Table 8. Retention trends of acidic/basic/neutral compounds based on structure and polarity ......................................................................................... 49

Table 9. Diamond Bidentate C18 Capacity Factor “ $k$ ” (ACN/ABN) .................. 49

Table 10. Bidentate C18 Capacity Factor " $k$ ” (ACN/ABN) ................................. 51

Table 11. Phenyl Hydride Capacity Factor " $k$ ” (ACN/ABN) ................................ 53

Table 12. Diol Capacity Factor "k” (ACN/ABN) .................................................. 55

Table 13. Diamond Bidentate C18 Capacity Factor “ $k$ ” (MeOH/Resveratrol) ... 58

Table 14. Bidentate C18 Capacity Factor “k” (MeOH/Resveratrol) .................... 59

Table 15. Phenyl Hydride Capacity Factor “k” (MeOH/Resveratrol) .................. 59

Table 16. Diol Capacity Factor “k” (MeOH/Resveratrol) ..................................... 60

Table 17. Diamond Bidentate C18 Capacity Factor " $k$ ” (MeOH/ABN) ............. 68

Table 18. Bidentate C18 Capacity Factor " $k$ ” (MeOH/ABN) ............................... 69

Table 19. Phenyl Hydride Capacity Factor “ $k$ ” (MeOH/ABN) ............................. 69

Table 20. Diol Capacity Factor “k” (MeOH/ABN) …………………………........ 69 


\section{LIST OF FIGURES}

Figure 1. Dual retention of an ANP compound and a RP compound.................. 6

Figure 2. A "U” shaped retention map of a hybrid hydrophilic/hydrophobic compound using an ANP stationary phase................................................... 7

Figure 3. Example of a Chromatogram..................................................... 13

Figure 4. Silica hydride synthesis......................................................... 22

Figure 5. Functionalization of silica hydride by hydrosilation......................... 23

Figure 6. Tested Column Structures........................................................ 25

Figure 7. Perkin Elmer Flexar UPLC ....................................................... 27

Figure 8. Agilent 1200 Series HPLC ...................................................... 28

Figure 9. Resveratrol Analogues ........................................................ 30

Figure 10. Acidic, Basic and Neutral Compounds ...................................... 31

Figure 11. Diamond C18 Retention Map (ACN/Resveratrol) ........................ 37

Figure 12. Diamond Bidentate C18 with Acetonitrile Mobile Phase Overlaid/Stacked Chromatograms (Resveratrol) ......................................... 39

Figure 13. Bidentate C18 Retention Map (ACN/Resveratrol) ......................... 40

Figure 14. Bidentate C18 with Acetonitrile Mobile Phase Overlaid/Stacked Chromatograms (Resveratrol) ...................................................................... 42

Figure 15. Phenyl Hydride Retention Map (ACN/Resveratrol) ....................... 44

Figure 16. Phenyl Hydride with Acetonitrile Mobile Phase Overlaid/Stacked Chromatograms (Resveratrol) ................................................................... 45

Figure 17. Diol Retention Map (ACN/Resveratrol) ................................... 46

Figure 18. Diol with Acetonitrile Mobile Phase Overlaid/Stacked Chromatograms................................................................................ 48 
Figure 19. Diamond C18 Retention Map (ACN/ABN)

Figure 20. Diamond Bidentate C18 with Acetonitrile Mobile Phase Overlaid/Stacked Chromatograms 50

Figure 21. Bidentate C18 Retention Map (ACN/ABN) 51

Figure 22. Bidentate C18 with Acetonitrile Mobile Phase Overlaid/Stacked Chromatograms 52

Figure 23. Phenyl Hydride Retention Map (ACN/ABN) 53

Figure 24. Phenyl Hydride with Acetonitrile Mobile Phase Overlaid/Stacked Chromatograms 54

Figure 25. Diol Retention Map (ACN/ABN) 55

Figure 26. Diol with Acetonitrile Mobile Phase Overlaid/Stacked Chromatograms 56

Figure 27. Diamond C18 Retention Map (MeOH/Resveratrol) 60

Figure 28. Diamond Bidentate C18 with Methanol Mobile Phase Overlaid/Stacked Chromatograms 61

Figure 29. Bidentate C18 Retention Map (MeOH/Resveratrol) 62 Figure 30. Bidentate C18 with Methanol Mobile Phase Overlaid/Stacked Chromatograms

Figure 31. Phenyl Hydride Retention Map (MeOH/Resveratrol) 64

Figure 32. Phenyl Hydride with Methanol Mobile Phase Overlaid/Stacked Chromatograms

Figure 33. Diol Retention Map (MeOH/Resveratrol) 66

Figure 34. Diol with Methanol Mobile Phase Overlaid/Stacked Chromatograms

Figure 35. Diamond Bidentate C18 with Methanol Mobile Phase Overlaid/Stacked Chromatograms 
Figure 36. Bidentate C18 with Methanol Mobile Phase Overlaid/Stacked

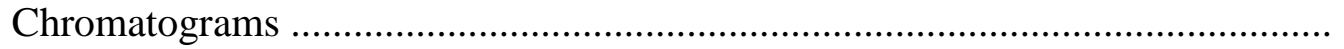

Figure 37. Phenyl Hydride with Methanol Mobile Phase Overlaid/Stacked Chromatograms

Figure 38. Diol with Methanol Mobile Phase Overlaid/Stacked Chromatograms 74

Figure 39. Diamond C18 Retention Map (MeOH/ABN) .............................. 75

Figure 40. Bidentate C18 Retention Map (MeOH/ABN) ................................ 75

Figure 41. Phenyl Hydride Retention Map (MeOH/ABN) ............................ 76

Figure 42. Diol Retention Map (MeOH/ABN) .......................................... 76 


\section{Chapter 1: Introduction}

\subsection{Background}

\subsubsection{History of Chromatography}

Chromatography is an analytical technique used to separate a mixture of components in a mobile phase based on various molecular interactions between a stationary phase and analytes of interest. ${ }^{1}$ This technique is mainly used qualitatively in the form of identifying components but it can also be used in a quantitative manner for determining precise amounts of each analyte. The use of chromatography dates back to the 1800s when Drs. Friedlieb Runge, Friedrich Goppelsroeder and Lester Reed separated various colored mixtures on paper and cloth. ${ }^{2,3}$ These three scientists are referred to as the pioneers of chromatography while the majority of the scientific community consider Dr. Michael Tswett as the father of the concept.

Michael Tswett was a Russian botanist who worked extensively with plant pigments such as chlorophyll. During the pigment sample preparation, he utilized calcium carbonate to prevent degradation and observed adsorption of all of the pigments except

for carotene. ${ }^{4}$ In the early 1900 s, these findings motivated Dr. Tswett to develop an analytical method for the separation of chlorophyll, xanthophyll and carotene. Tswett utilized column chromatography in which calcium carbonate adsorbent was packed into a glass column followed by the addition of petroleum ether chlorophyll solution as mobile phase. As the mobile phase with chlorophyll passed through the adsorbent phase, the pigments would separate based on their affinity to the calcium carbonate, generating a chromatogram that showed distinct colored bands. 


\subsubsection{Types of Chromatography}

1.1.2.1 Homogeneous vs. Heterogenous. Since the 1900s, the utilization and popularity of chromatographic separations have grown and branched into different categories. While there are numerous types of chromatography, the three mentioned here depend on: (1) different physical states, (2) types of interaction between analyte and mobile/stationary phase and (3) the shape of stationary phase. ${ }^{5}$ Chromatography distinguished by physical states are homogenous (same phases) and heterogenous (different phases) chromatography. Homogenous chromatography separates compounds through solute distributions between two liquid phases, one mobile and the other stationary. Affinity for each phase determines the solute distribution similar to the concept of liquid-liquid extraction. Common heterogeneous forms of chromatography are gas and liquid chromatographies (GC and LC) which both incorporate a solid stationary phase. A column or capillary bed is packed with small solid particles and a gas or liquid mobile phase is passed through the stationary phase. Analytes with varying polarity will interact less or more strongly with the stationary phase where stronger interactions result in longer retention times.

1.1.2.2 Adsorption vs. Partition. Chromatography can also be categorized according to several types of interactions between the solute and mobile/stationary phase where partitioning and adsorption are considered the most common. In liquid chromatography, an immobilized liquid can act as a stationary phase, allowing for partitioning to occur. For example, water can form hydrogen bonds with a silica support which forms an immobilized liquid monolayer covering the stationary surface. As the mobile phase 
carries analyte through the column or capillary, the analyte will tend to stay in the liquid phase that shares a higher affinity. If the immobilized liquid is more polar than the mobile phase, then polar analytes with retain longer during the run. The simplest example of partitioning chromatography would be performing a liquid-liquid extraction where two immiscible liquids are forcefully mixed to separate polar and nonpolar solutes. The polar solutes would distribute in the aqueous phase while the nonpolar would distribute into the organic phase based on their partition coefficient.

The concept of adsorption also depends on affinity and polarity similarly to partitioning. The analytes in the mobile phase will interact with the solid stationary phase strongly or weakly depending on the polarity of the solid support. If the solid stationary phase is more polar than the mobile phase, then polar analytes will adsorb and be retained longer than less polar analytes. ${ }^{6}$ Functionalization of the columns and capillaries with moieties that vary in polarity will alter the partitioning and adsorption effects during chromatographic separations. For example, carbon chains with increasing length will decrease the polarity of the solid support while the addition of alcohols or carboxylic acids will increase the polarity.

1.1.2.3 Columnar vs. Planar. Another way to distinguish types of chromatography is based on the shape of the stationary phase. Columnar and planar chromatography are the most commonly shaped solid supports. In column chromatography, the stationary phase is cylindrically shaped and packed with adsorptive material. On the other hand, planar chromatography utilizes flat stationary phases that are either entirely made up of adsorbent or layered onto an inert flat surface. Examples of column chromatography are 
GC and LC/high-performance LC (HPLC) while planar includes paper, thin-layer chromatography (TLC) and high-performance TLC (HPTLC). ${ }^{7,8}$

\subsubsection{Main Chromatographic Modes}

As mentioned before, the stationary phase in chromatography can be modified to establish control over the desired separation. This control allows scientists to operate under different modes of retention. For the sake of this research project, the four main modes that will be distinguished and discussed are: (1) normal phase (NP), (2) hydrophilic interaction (HILIC), (3) reversed-phase (RP) and (4) aqueous-normal phase (ANP) chromatography. Each mode can be distinguished by the type of stationary phase and mobile phase used, along with their interaction with analytes of interest.

1.1.3.1 Normal Phase. NP chromatography incorporates a polar stationary phase and a non-polar mobile phase in order to retain and separate polar compounds. Examples of polar stationary phases would be bare silica (Si-OH) and functionalized solid support with polar moieties such as alcohols, carboxylic acids, amino and cyano groups. Examples of non-polar mobile phases used in NP mode would be hexane and tetrahydrofuran (THF). The mobile phase may be mixed with other moderately non-polar solvents such as chloroform, isopropanol and ethyl acetate to alter the retention of the non-polar analytes. In this mode of chromatography, non-polar compounds would elute first, followed by moderately non-polar and finally polar compounds.

1.1.3.2 Hydrophilic Interaction (HILIC). HILIC is named based on the hydrophilic or "water loving” interaction between the analytes and the mobile/stationary phase. This technique is used to separate polar and ionic species in a mixture. The HILIC 
chromatographic mechanism operates in the same manner as NP mode where non-polar compounds elute first followed by polar ones but differs in mobile phases. While HILIC uses polar solid supports, similarly to NP chromatography, the mobile phase differs by incorporating a mixture of organic and aqueous solvents. The organic mobile phases used in HILIC are more polar than hexane, typically used in NP and are miscible with water. Typical solvents used are methanol, acetonitrile and isopropanol. HILIC separations differ from NP by utilizing partitioning effects to separate analytes in a mixture.

\subsubsection{Reversed Phase. The next mode is referred to as reversed-phase} chromatography and is currently the most popular mode of separation. Named for operating in an opposing manner to NP chromatography, this technique retains non-polar compounds and elutes the polar ones. RP stationary phases typically incorporate hydrocarbon chains and rings such as C8, C18 and phenyl. Increasing the length of the carbon chain will increase the hydrophobicity of the column such that C18 columns demonstrate more retention of non-polar solutes than C8 columns. The mobile phases used in RP chromatography are like HILIC where water soluble organics are used, albeit higher proportions of water is used to elute the polar compounds earlier during analysis.

\subsubsection{Aqueous-Normal Phase. Last to be discussed is aqueous-normal phase} chromatography (ANP) which is unique to the former modes mentioned. ANP is a technique that requires the use of a special variety of silica particle, known as Type-C. The surface of Type-B silica particles is made up of Si-OH groups while Type-C silica particles incorporate silica hydride (Si-H). More information regarding these particles

will be discussed in Section 4. The mobile phases used in ANP mode are similar to those 
used in HILIC mode. While the mechanism of ANP has not been fully understood, this type of chromatography has demonstrated both NP-like and RP modes of separation. ${ }^{9}$

Given two compounds, one with hydrophilic characteristics and the other with hydrophobic ones, both can be retained and separated by altering the mobile phase composition from high to low organic or vice versa. Figure 1 below shows a retention map for an ANP compound and a RP compound on an ANP column. These stationary materials allow for more flexible analysis of hydrophilic and hydrophobic mixtures where at higher organic content non-polar solutes will elute first leaving polar solutes last. The opposite occurs when higher aqueous solvent is used to elute polar compounds before the non-polar ones. Another unique ability of the ANP mode is when a compound has both hydrophilic and hydrophobic properties the retention map at varying mobile phase compositions forms a "U" shape (Figure 2).

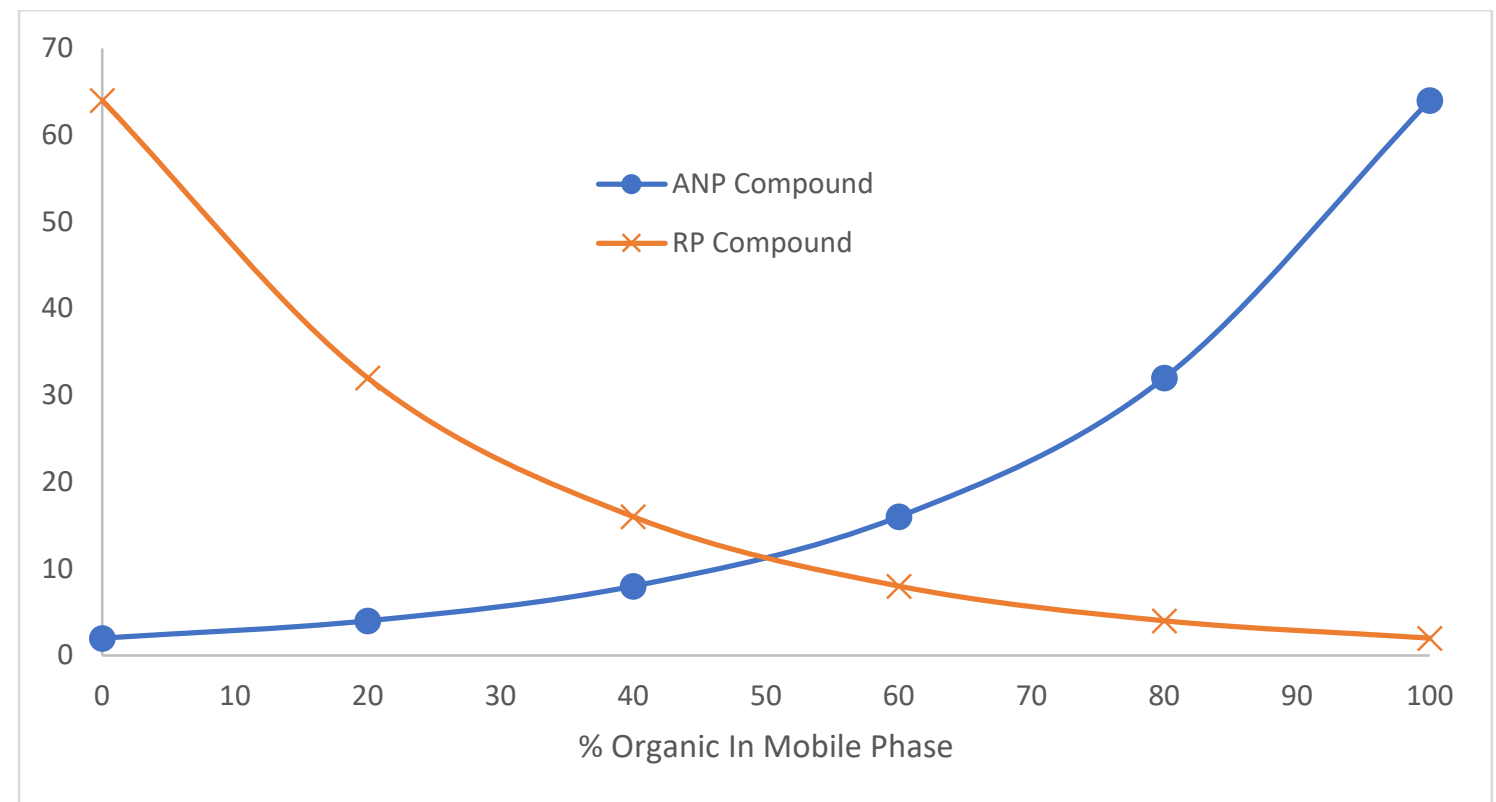

Figure 1. Dual retention of an ANP compound and a RP compound. The ANP compound retains as organic mobile phase composition increases while the RP compound retains at lower organic percentages. 


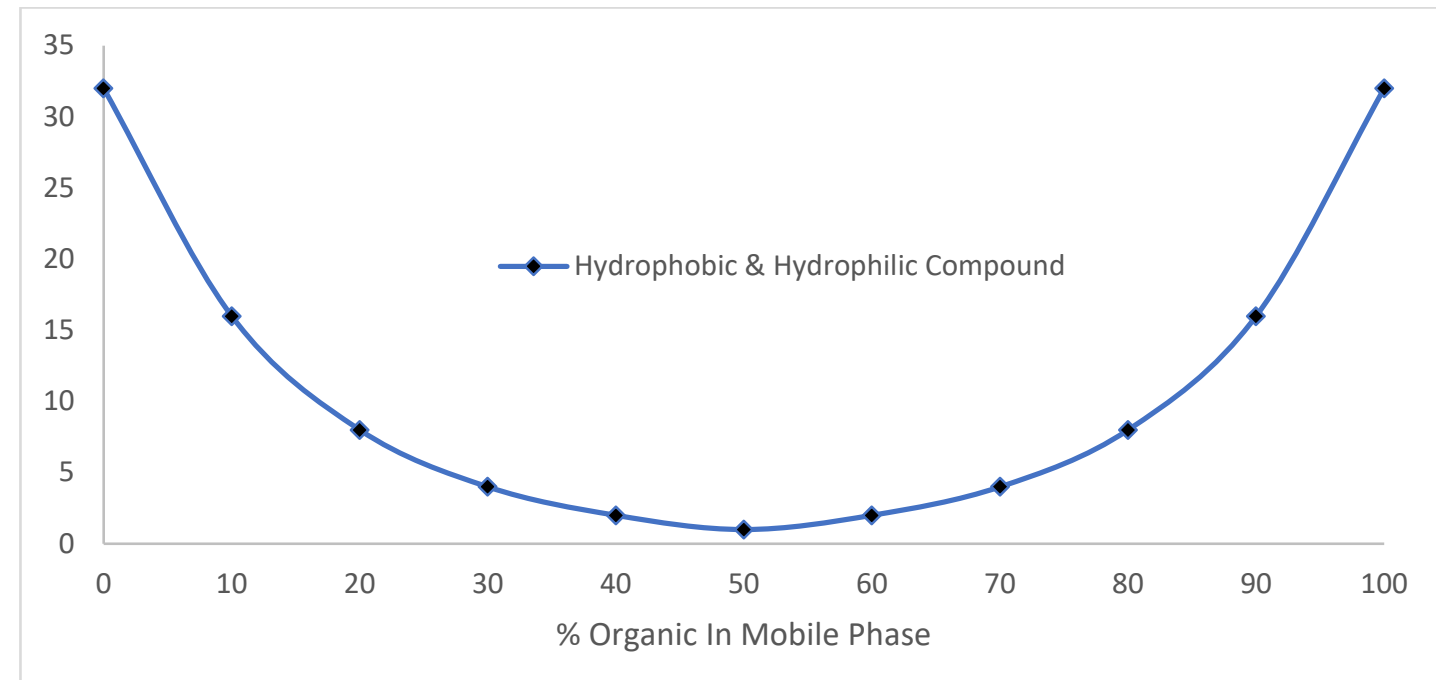

Figure 2. A "U" shaped retention map of a hybrid hydrophilic/hydrophobic compound using an ANP stationary phase. This graph demonstrates the effect of ANP mode on compounds with hybrid hydrophobicity such as peptides. Retaining strongly at low organic, weakly at $50 \%$ and strongly again at high organic.

1.1.3.5 Other Modes of Separation. There are many other forms of chromatography such as ion exchange (IEC) and size exclusion (SEC). The former focuses on separating charged species while the latter is based on molecular size. Ion exchange can be separated into three categories: 1 . cation-exchange, 2. anion-exchange and 3. zwitterionic-exchange. The concept of ion exchange is based on weakly bound ions in solution trading places with stronger ionic species. Stationary phases for this chromatography incorporate covalently bound polymers with ionic groups. Mobile phases contain buffers dependent on the stationary phase used such that cation-exchange supports will require cationic buffers. The cations in solution will bind to the covalently bound anions and once a stronger cation approaches; coulombic forces will dictate the exchange of ions. This same phenomenon will occur with anions and zwitterions differing only with stationary and mobile phases used. The example below shows the exchange between the weak ions $\left(\mathrm{B}^{+/}\right)$and the strong ions $\left(\mathrm{C}^{+/}\right)$. 
1. $R-A^{+/-}-B^{+/-}+C^{+/-}$(Before ion exchange occurs)

2. $R-A^{+/-}-C^{+/-}+B^{+/-}$(After ion exchange occurs)

(" $\mathrm{R}$ " is the polymer bound to solid support and " $\mathrm{A}$ "-»" is the permanent ion attached to the polymer.)

SEC has several different forms but the two most common in this field are gel filtration (GFC) and gel permeation (GPC). This type of chromatography is typically used for macromolecule separation. Both GFC and GPC operate in a similar manner where the mobile phase, aqueous for GFC and organic for GPC, carries analytes of various sizes through a column with porous particles. Analytes that are smaller than the pores of the stationary phase will spend more time traversing through the numerous pathways than larger molecules. Since the larger molecules bypass the pores, their overall pathlength is shorter leading to earlier elution.

\subsection{High Performance Liquid Chromatography}

Liquid chromatography has undergone vast improvements since its discovery. In the early 1940s, Drs. Martin and Synge proposed the idea that to increase efficiency of separations smaller particles and higher pressures needed to be implemented. ${ }^{10}$ This idea continued to gather support and lead to the birth of high pressure liquid chromatography. The word "pressure" in the name of this technique was later changed to "performance" as the first HPLC systems were commercialized in the late 1960s. ${ }^{11}$ To date, modern HPLC has advanced to ultra-performance liquid chromatography (UPLC or UHPLC) which allows stable analysis at pressures greater than 1000 bar. ${ }^{12}$ This analytical technique offers advantages such as improved qualitative/quantitative analyses, reproducibility, high sensitivity, high resolution of molecular mixtures and increased cost efficiency. The 
rapid growth of HPLC has made it one of the most popularly used analytical techniques and covers a wide range of applications.

\subsubsection{Applications in Modern HPLC}

Of many applications, five frequently utilize the efficiency and precision of HPLC. The first are pharmaceutical and bioanalytical separations such as quality control (QC) and compound purification. The advantages mentioned before, allow QC analysts to separate and verify precise concentrations of compounds in compliance with FDA guidelines and regulations. For example, separation of metabolites in medicinal plants is necessary for Good Manufacturing Practices and requires robust separation techniques to quantify specific compounds. ${ }^{13,14}$ Next is within the field of forensic science, where HPLC's specificity and sensitivity are used to solve a variety of criminal cases. Examples of applications in forensics include detection of squalene from fingerprints, distinguishing ballpoint pen ink and detection of illegal substances in biological fluids such as narcotics and amphetamines. ${ }^{15,16}$

Food science is another popular area due to society's interest in determining both health beneficial and detrimental substances in consumable goods. HPLC allows food scientists to quantify favorable compounds such as flavonoids, polyphenols, antioxidants, minerals and amino acids rapidly and efficiently. On the other hand, they can also monitor carcinogens, pesticides and steroids with extremely high sensitivity (femtomoles). ${ }^{17-19}$ The fourth useful application of HPLC is in environmental analysis of soil and water samples. Similar to the pharmaceutical and forensic fields, the sensitivity of this analytical technique allows for proper regulation of neurotoxins, herbicides and 
other environmental/ecological contaminants. ${ }^{20-22}$ Last but not least, is the utilization of HPLC in research and development. Whether it be for qualitative or quantitative information, HPLC is essential for most laboratories because of the precision, accuracy, reproducibility and sensitivity that it boasts. Technological and engineering advancements of the HPLC system and components has impacted the speed, efficiency and cost of sample analysis worldwide.

\subsubsection{Instrument Components}

There are five major components to an HPLC system: 1. Solvent Manager, 2. Injector, 3. Column/Column Oven, 4. Detectors and 5. Computer/Software.

1.2.2.1 Solvent Manager. The solvent manager consists of a solvent reservoir to hold several reagent bottles and pumps to force mobile phase to flow from the bottles throughout the system. Additionally, solvent managers may incorporate degassing to remove dissolved oxygen, pulse dampeners to absorb pump pressure fluctuations and solvent mixers allow the use of gradient methods. The most common solvents used in HPLC are deionized water, acetonitrile, methanol, tetrahydrofuran, isopropanol and ethanol. Factors to consider when choosing a particular solvent are the viscosity, inertness and miscibility with other solvents. Highly viscous solvents require more pressure to pass through the system which may lead to pump failure, system leaks, column damage, inconsistent peak shape or false peaks. Inert solvents will not interact with dissolved solutes preventing interference between them and the stationary phase. Solvents that are miscible with each other allow for consistent elution during analysis. Additionally, the ultraviolet (UV) cut off range is important because UV detectors 
(mentioned later) are common in HPLC practice. The UV cutoff is important because solvent that absorbs UV light will interfere with samples analyzed at specific wavelengths. For example, the UV cut off for acetonitrile and methanol is $210 \mathrm{~nm}$ while ethyl acetate's is $260 \mathrm{~nm}$ making acetonitrile/methanol a better solvent choice when analyzing compounds between $210-260 \mathrm{~nm}$. The ideal solvent manager is capable of delivering solvent at a variety of flow rates $(0.1-5 \mathrm{~mL} / \mathrm{min})$, operating at high pressures and generating a stable baseline.

\subsubsection{Injector. The injector component incorporates a sample plate or tray, a fine} needle and an injection loop. An ideal injector unit is capable of picking up precise amounts of sample and introducing that sample to the mobile phase in an accurate and highly reproducible manner. Typical HPLC systems use sample plates or trays that hold vials (removable) or wells (fixed) containing small volumes of sample solution. The needle or syringe picks up a precise amount of solution from the vial/well, usually 1 to $100 \mathrm{uL}$, which is then transferred (manually or automated) to an injection loop that holds the sample prior to introduction into the mobile phase. Two common types of injection loops are fixed loops where an exact amount of sample completely fills the loop, and partial loops hold a small amount of sample with the remaining volume consisting of the mobile phase. The sample held in the loop is then introduced to the mobile phase as a rectangular "plug" by a switching valve and flows in a laminar fashion. Ideal resolution of the plug gives a perfect Gaussian profile; although viscosity of sample/solvent, pressure variance and injection of large sample volumes may be detrimental to the peak shape. 
1.2.2.3 Column. A typical chromatographic column is made up of an outer cylindrical tube, frits and solid packing material. The tube is made of inert material such as stainless steel or chemically/physically strong plastic such as polyetheretherketone (PEEK). Dimensions of an HPLC column range from $30-250 \mathrm{~mm}$ in length and $0.05-10 \mathrm{~mm}$ for internal diameter. Analytical columns can be classified based on the size of internal diameter from standard ( $3-4.6 \mathrm{~mm})$ to nanoscale (0.05 - $0.2 \mathrm{~mm})$. Modern HPLC advancements are moving towards smaller columns due to the use of smaller packing material. Reasons for developing smaller particles will be discussed in the parameters section below. The column frits are small porous disks made with similar inert material as the cylindrical tube but are positioned between the packing material and the entrance and exit of the column. Frits are designed to allow mobile phase to pass through the column and prevent the stationary phase from leaving. The most important part of the column is the packing material which is often composed of small spherical and inert particles (Si-OH, Si-H, Polymer). The two main physical features of these particles are surface area and particle size which both contribute to the retention ability of the column. There are two types of particles, porous and core-shell (solid core and porous outer layer) which typically vary in size from $3-5 \mathrm{um}$. An active phase can be chemically or physically bound to the bare particles generating a variety of columns such as the C8, C18, phenyl, amino, etc. Different active phases will demonstrate varying stability, chemical nature and surface reactivity making their effect on molecular separations unique to each other. Additional components can be coupled to enhance or protect a 
column such as a column oven (allowing temperature control) and guard columns (block highly retaining particles).

1.2.2.4 Detectors. Sample detection is necessary in all fields of analytical chemistry to exploit physical/chemical properties presenting qualitative and quantitative information of analytes. Ideal detectors should be extremely sensitive, give reproducible response, cause minimal peak broadening and maintain robust stability under flow/pressure variance. Detectors used to measure analyte concentration in HPLC convert an electrical signal into a chromatogram. The chromatogram below (Figure 3) displays an intensity (arbitrary units) against time, resulting in a peak unique to the analyte in question.

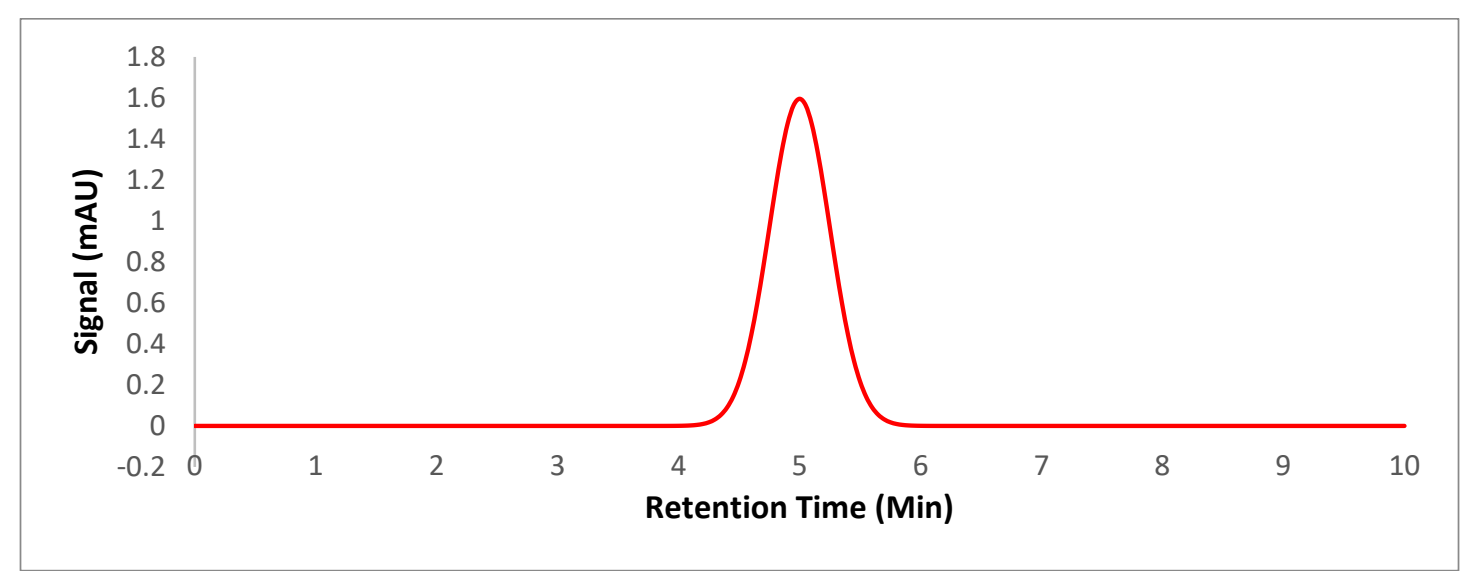

Figure 3. Example of a Chromatogram.

Area under the Gaussian curve is then calculated and compared to oscillating electrical output, also known as a signal to noise ratio (acceptable when signal/noise $>3$ ). There are numerous detectors used in HPLC analysis but this paper will focus on UVvisible and MS detectors. UV-vis detectors utilize a light source that produces monochromatic light (200 - $600 \mathrm{~nm})$ directed through a flow cell. The light is then split, where one portion interacts with sample in solution and the second goes directly to a 
reference detector. Interaction between specific wavelengths $(\lambda)$ of light and sample in solution can be measured based on transmittance ( $T$ ) and absorbance $(A)$. Transmittance is the relation between intensity of light passing through a blank solution (no analyte) vs. intensity transmitted through a sample solution (Equation 1). Absorbance is the logarithm $\left(\log _{10}\right)$ of the inverse of transmittance (Equation 2) and is necessary to calculate the molar concentration $(\chi)$ according to the Lambert-Beer law (Equation 3). By rearranging the Lambert-Beer law and utilizing calibration and/or standard addition curves quantification of light absorbing compounds is possible. Variable-wavelength detectors such as photodiode array detectors (PDA) or diode array detectors (DAD) allow for analysis at multiple wavelengths at one given time. PDA/DADs are extremely useful in determining optimal wavelengths in unknown sample solutions but are considerably more costly than single wavelength detectors.

Eq. 1. $T=I_{1} / I_{0}$

Eq. 2. $A=\log _{10}(1 / T)$

Eq. 3. $A=\varepsilon_{\lambda}[\chi] L$

Mass spectrometers are another form of detectors that continue to grow in popularity and common practice due to their high sensitivity and selectivity. MS detectors are used to distinguish molecules based on their mass to charge ratio $(\mathrm{m} / \mathrm{z})$ and do not necessarily need to resolve a set of peaks to do so. By focusing on a precise $\mathrm{m} / \mathrm{z}$, one can differentiate between compounds that coelute. The ionization of molecules is of utmost importance for MS detection. In LC-MS, soft ionization techniques such as electrospray ionization (ESI) and atmospheric pressure chemical ionization (APCI) are employed. Both techniques 
seek to ionize molecules of interest while leaving solvent molecules uncharged but each form ions differently. ESI incorporates an electrically charged (3-5 kV) capillary carrying mobile phase and analyte which is nebulized into fine droplets. The droplets are then vaporized by heated gas, evaporating most of the solvent and eventually ionic repulsion leads to the formation of individual ions. APCI operates with a heated capillary which vaporizes the mobile phase and analytes before inducing charge. The gas formed after heating is then mixed with $\mathrm{N}_{2}$ or $\mathrm{O}_{2}$ gas and forced to pass a corona discharge electrode/needle (3-5 kV). Charges on vaporized solvent and added $\mathrm{N}_{2} / \mathrm{O}_{2}$ migrate to the analytes based on differences in polarity. The molecules can be positively or negatively charged depending on their gas phased basicity (GPB) or acidity (GPA). The tendency for molecules to gain a proton (amines) follows the GPB phenomenon and the tendency to lose a proton (carboxylic acids, carbohydrates) coincides with GPA. These concepts can be characterized by free enthalpy change where $\Delta \mathrm{G}_{\mathrm{GPB}}^{0}<\Delta \mathrm{G}_{\mathrm{GPA}}^{0}$ (most molecules) and supports that positive ions form more easily in comparison to negative ones. The individual ions are then funneled to a mass analyzer such as a quadrupole, ion trap or time of flight (TOF). Resolution of $\mathrm{m} / \mathrm{z}$ is lower with single mass analyzers because the molecular ions formed do not tend to fragment. Coupling mass analyzers together allows for tandem MS analysis (LC-MS/MS) where parent ions are first isolated, fragmented in a collision cell and finally analyzed based on daughter ions. Various LC-MS/MS systems demonstrate high resolving power also known as the ability to distinguish between two peaks. 
1.2.2.5 Computer and Software. The last component to be discussed is the computer and software which convert the electric signal produced from the detector into a chromatogram. The data is collected and saved for further analysis depending on the needs of the chemist. Software is proprietary and usually distributed with similar branded instrumentation but some software may be compatible with varying manufactured instruments. Typical software allows an analyst to prepare methods and sequences, adjust system parameters (temperature, flow rate, mobile phase composition, injection volume, etc.) and analyze data. Data analysis generates information such as retention time, peak width, peak height, peak area and $\mathrm{S} / \mathrm{N}$ ratios. Modern programs offer auto-integration of peak areas but also allow the user to manually integrate when necessary.

\subsection{HPLC Parameters}

\subsubsection{Resolution}

Of the many equations that are associated with HPLC, there are two main ones, resolution (R) and the Van Deemter equation. The former calculates the ability to distinguish between any two peaks during a run, while the latter accounts for peak broadening. Resolution consists of three parameters which are: retention factor $(\mathrm{k})$, selectivity $(\alpha)$ and efficiency $(N)$. Equation 4 shows the simplest way to define the resolution where only retention time and peak width are taken into account while Equation 5 incorporates retention factor, selectivity and efficiency. Good resolution is associated with complete peak separation, where $\mathrm{R}>1$ and can be controlled by altering the other parameters.

$$
\text { Eq. 4. } R=\frac{2\left[t_{R}(A)-t_{R}(B)\right]}{W_{b}(A)+W_{b}(B)}
$$


Eq. 5. $R=\frac{1}{4}\left(\frac{\alpha-1}{\alpha}\right)\left(\frac{k}{1+k}\right) \sqrt{N}$

1.3.1.1 Retention Factor. The retention factor "k", also known as capacity factor, is needed to characterize specific peak retention and is independent of flow rates and column dimensions. One way to describe the retention factor is as a ratio between reduced retention time $\left(\mathrm{t}_{\mathrm{R}}-\mathrm{t}_{0}\right)$ and the dead time $\left(\mathrm{t}_{0}\right)$, shown in Equation 6. The retention time corresponds to the analyte of interest while the dead time refers to an analyte that has no affinity for the stationary phase. For example, $\mathrm{KNO}_{3}$, a polar inorganic salt, will not retain on nonpolar stationary phases such as C8 or C18 functionalized columns. Dead time can also be related to dead volume which is described as the volume of mobile phase that passes through a column from the beginning of an injection. Retention factors are positioned between 2 and 10, where compounds that demonstrate a value less than 2 indicate poor retention and a value above 10 shows that the compound is too strongly retained.

$$
\text { Eq. 6. } k=\frac{t_{R}-t_{0}}{t_{0}}
$$

1.3.1.2 Selectivity. The selectivity parameter " $\alpha$ " compares the retention of multiple analytes in solution. Selectivity can be controlled by altering mobile and stationary phases but is also specific to the nature of the solute. Consider two analytes A and B in solution are injected into an HPLC system and separated by a chromatographic column. The selectivity of these two analytes is dependent on the ratio of their retention times where a higher $\alpha$ value indicates greater separation (Eq. 7 below). Since selectivity is observed from the apex of peaks, regardless of how high $\alpha$ is determined to be, peak broadening may be large enough to prevent full separation. 


$$
\text { Eq. 7. } \alpha=\frac{t_{R_{B}}}{t_{R_{A}}} \quad \text { (Assumes } t_{\mathrm{RB}}>\mathrm{t}_{\mathrm{RA}} \text { ) }
$$

1.3.1.3 Efficiency. The last characteristic parameter in controlling resolution is efficiency which can be expressed as a number of theoretical plates " $N$ ". A theoretical plate can be described as a fraction of column where an equilibration event occurs between the mobile and stationary phases. $N$ is directly related to peak broadening and is used to determine a column's efficiency where high values for $N$ give narrower peaks. The plate number is derived from the height equivalent of a theoretical plate (HETP) which relates peak variance to the length of the analytical column. Two ways to solve for $N$ are shown below in association with column length or retention time.

$$
\text { Eq. 8. } N=L / H \quad \text { or } N=16 \sqrt{\frac{t_{R}}{W_{b}}}
$$

\subsubsection{Van Deemter Equation}

Even though HPLC systems utilize high pressure pumps to push mobile phase through the column at constant flow rates, diffusion and the kinetics related to the process still transpire. The Van Deemter equation accounts for all possible forms of diffusion occurring within the column and stationary phase, consisting of five types: longitudinal, eddy, lateral movement due to convection, mass transfer in/out of the stationary and stagnant mobile phase diffusions. Equation 9 below shows how each form of diffusion contributes to the height of a theoretical plate.

$$
\text { Eq. 9. } H=H_{L}+H_{E}+H_{C}+H_{T}+H_{S}
$$

The first and most common form in HPLC is longitudinal diffusion which occurs when a concentrated region (in liquid or gas) disperses to equally fill the surrounding area 
over time. Longitudinal diffusion through the column depends on several factors such as particle obstruction (silica bed), the linear flow rate, the nature of solvent and the dimensions of the column/packing material. Second, is eddy diffusion which takes place due to the groups of molecules spreading out within the stationary phase and following different paths. This type of diffusion is directly related to how well the column is packed and how uniform the particles are. The main factors in determining the effects of eddy diffusion on plate height are column packing and average particle diameter.

The next form is the first of three mass transfer diffusions and refers to the lateral movement of material caused by convection of the mobile phase. The convection of molecules in the mobile phase during HPLC analyses is directly related to particle diameter, velocity of mobile phase and the diffusion coefficient. Fourth is the mass transfer of solutes in and out of the stationary phase. The particular mass transfer depends on adsorption/desorption kinetics between the solute and stationary, the morphology of the packing material (i.e. shape, thickness) and the diffusion coefficient. Finally, the last form of mass transfer diffusion needing to be considered is the transfer of solute in/out of stagnant mobile phase within porous media. This method of diffusion is governed by the particle shape, pore depth and the diffusion coefficient. Combining all the parameters provides the Van Deemter equation, relating each type of diffusion with linear velocity and HETP where constant "A" accounts for eddy, " $\mathrm{B}$ ” for longitudinal and " $\mathrm{C}$ ” for mass transfers (combines convection, in/out of stationary and in/out of stagnant mobile phase). ${ }^{23}$

$$
\text { Eq. 10. } H=A+\left(B \times \frac{1}{u}\right)+(C \times u)
$$




\subsection{Stationary Phase - Silica}

\subsubsection{Advantages}

Inorganic, polymer and hybrid materials have been researched for solid support foundations in the synthesis of HPLC stationary phases. Of these materials, inorganic particles such as silica, metal oxides, graphite and hydroxyapatite are most commonly used in modern applications. ${ }^{24}$ Silica demonstrates characteristic advantages making silica the most popular choice for adsorbent material. Three main advantages are mechanical robustness, chemical/thermal stability and flexible control of surface area, pore size and pore diameter. Mechanical strength is tested by increasing pressure and observing any variance in flow rates due to uniformity change or particle damage. The chemical and thermal stabilities are determined by exposing the materials to different $\mathrm{pH}$ and temperature environments followed by efficiency testing. Lastly, the surface area, pore size/diameter can be characterized with scanning electron microscopy (SEM), transmission electron microscopy (TEM) and nitrogen adsorption studies. ${ }^{25}$ Even with these beneficial characteristics, silica has been continuously improved over the years.

\subsubsection{Evolution of Silica}

The first silica particles (Si-OH) used as adsorbent material for HPLC were not made uniformly and varied in size and shape. These "irregularly shaped silica” materials gave little reproducible data and limited scientists to using NP chromatography. Another issue with the irregular shape is the susceptibility to produce fines under mechanical stress. Particle fines can accumulate and result in unstable packed beds and column/system clogging. These issues lead to the production of spherical and uniformly dimensioned 
silica via a sol-gel process, also referred to as type A. Type A silica provided evenly packed beds which increased column lifetime and stability. Despite this advancement, trace metals found in the silica framework prevented proper solute-silanol interaction when solutes were ionized. The desire to decontaminate type A silica directed the chromatographic community towards the high purity form, type B silica. Type A silica was purified through acid treatments added to the sol-gel process. Type B silica demonstrated improved peak shapes and further increased mechanical/chemical stability. Even with the progression from irregularly shaped to high purity type B silica, the residual silanol groups left analysts with several issues.

These free silanols may be detrimental for separations if they electrostatically interact with solutes. Scientists have tried end-capping with methyl groups to reduce this issue but in doing so limited the $\mathrm{pH}$ range for analysis $(\mathrm{pH} 5-8)$. Lower $\mathrm{pH}$ will lead to the hydrolysis of these end caps and higher $\mathrm{pH}$ results in dissolution of the silica bed. Another problem that arises during analysis using type-B silica is an accumulation of adsorbed water. If water is present in the mobile phase, over the course of a run, a quasiliquid layer will form and grow with further hydrogen bonding resulting in partitioning effects. The effects of partitioning are detrimental in gradient analyses (variation of mobile phase composition with time) and equilibration times. ${ }^{26}$ The solution to these issues was the development of Type-C silica which incorporates a Si-H particle surface instead of Si-OH. Since there are minimal amounts of Si-OH groups $(<5 \%)$ the monolayer of adsorbed water is almost nonexistent. In addition to this improvement, Type-C silica maintains the uniform shape and high purity that type-B has to offer 
without needing end-capping to operate in standard $\mathrm{pH}$ ranges (2.5 - 7.5). Another unique capability mentioned before in Figures 1 and 2, is the use of the ANP mode (selective to Si-H stationary phases) for dual retention on a single column. Type-C silica has numerous applications supporting its growth as the next generation of silica materials.

\subsection{Synthesis of Si-H}

\subsubsection{Silanization}

For the synthesis of silica hydride materials, two silanization reactions were tested. The first involves converting the silanols to Si-Cl by reacting with thionyl chloride. Reduction from Si-Cl to Si-H is carried out with lithium aluminum hydride. One constraint with this reaction is that it must be performed in a completely dry environment. Since the Si-Cl moieties are unstable in the presence of water, they are likely to return to their former $\mathrm{Si}-\mathrm{OH}$ form. The second reaction is the condensation of the silanols to the hydride form through interaction with triethoxysilane. Unlike the first reaction, this one is not as sensitive to water and even utilizes an aqueous acid catalyst. A schematic of the reaction is shown below in Figure 4.

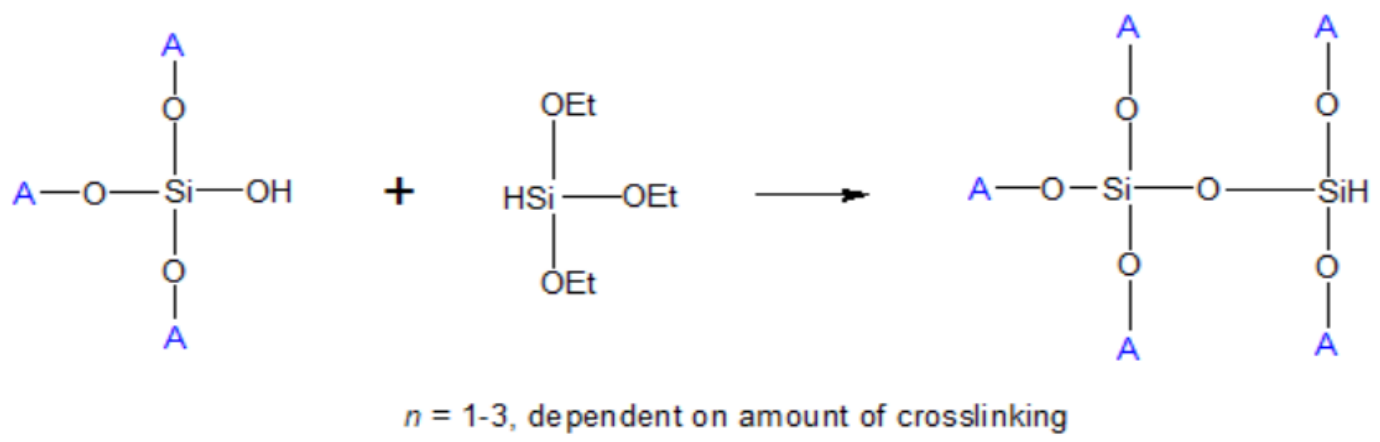

Figure 4. Silica hydride synthesis. Silanization performed on Si-OH materials with triethoxysilane to yield $\mathrm{Si}-\mathrm{H}$ materials. 


\subsubsection{Hydrosilation}

Hydrosilation offers a wide array of attachment options outside of typical stationary phase functionalization. The best example is the formation of doubly bound carbon when an alkyne is used instead of an alkene. The carbon forms a single bond to two Si-H neighbors giving high stability bidentate ligands on the stationary surface. Figure 5 shows the basic reaction scheme for hydrosilation. ${ }^{27}$

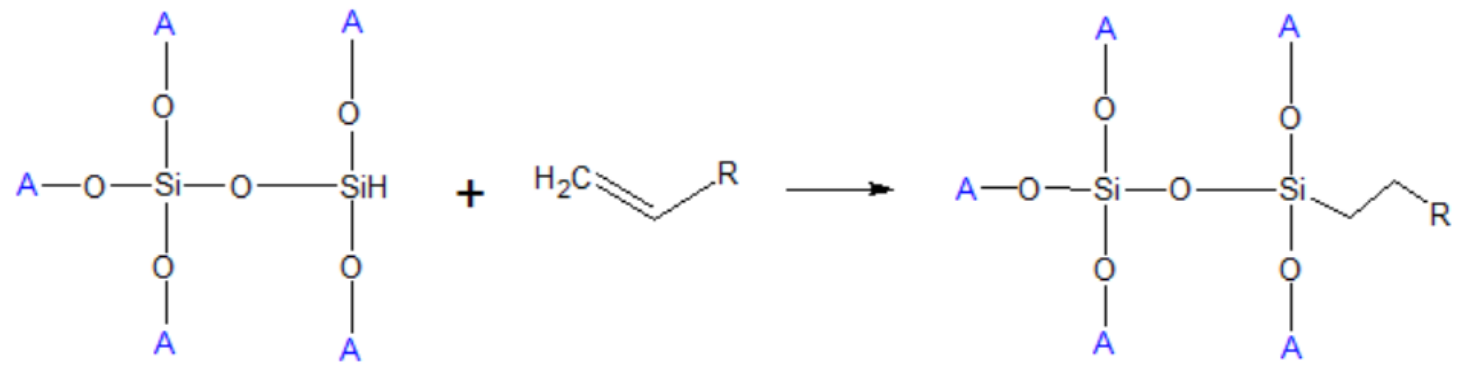

Figure 5. Functionalization of silica hydride by hydrosilation. An alkene (or alkyne) is reacted with silica hydride materials in the presence of hexachloroplatinic acid catalyst giving a silicon-alkyl (two silicon-alkyl bonds if an alkyne was used) covalent bond.

\subsection{Research Aims and Goals}

This thesis project is aimed towards the characterization of four Type-C silica columns through the analysis of retention maps based on resveratrol analogues. Resveratrol, a phenolic compound commonly found in red wines, grape skin and other berries has been associated with health beneficial effects such as fighting heart disease and cancer. ${ }^{28-30}$ Although these benefits are attractive, resveratrol has limitations such as solubility issues and poor bioavailability. ${ }^{31}$ Two solutions would be to derivatize resveratrol or to use other analogues with similar health benefits. ${ }^{32,33}$ The latter solution is most relevant to this research, in that the separation of the resveratrol analogues is important for future work in characterizing them individually. In addition, compounds of 
similar structure are more challenging to separate and by achieving separation allow for more detailed analysis of each column's retention capabilities.

The four columns (Diamond C18, Bidentate C18, phenyl and diol) share a silica hydride surface yet are uniquely functionalized. The Diamond C18 and Bidentate C18 columns are expected to exhibit retention on all the non-polar compounds. The diol column is expected to retain polar compounds while the phenyl functionalized column should interact more with those compounds with higher degrees of unsaturation. The resveratrol analogues were analyzed using UV detectors coupled to HPLC instruments; observed wavelengths ranged from $220 \mathrm{~nm}$ to $335 \mathrm{~nm}$. Solvents used were acetonitrile with $0.1 \%$ formic acid, deionized water with $0.1 \%$ formic acid and methanol with $0.1 \%$ formic acid (all solvents were at least HPLC grade). An additional study was performed observing retention of a small set of acidic, basic and neutral compounds. Future studies may include varying $\mathrm{pH}$ or temperature and analysis of folic acid derivatives. 


\section{Chapter 2: Experimental and Methods}

\subsection{Columns}

Four Type-C silica columns were used for this project: Bidentate C18, Diamond C18, phenyl hydride and diol (Figure 6).
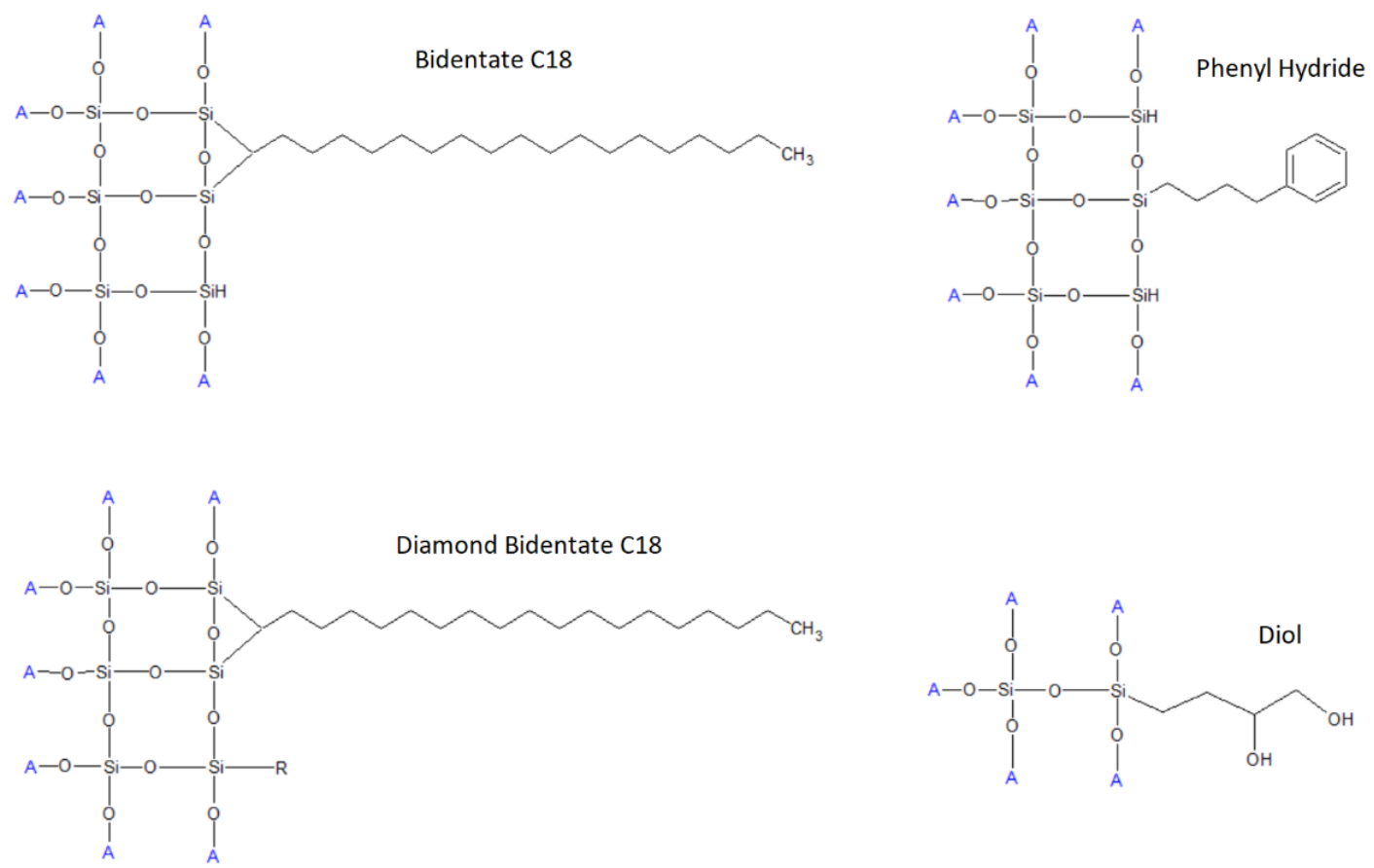

Figure 6. Tested Column Structures.

The Bidentate C18 incorporates a chain of 18 carbons with two covalent bonds to two neighboring silicon atoms, making the column extremely robust in comparison to other C18 columns. In addition to RP mode, the Bidentate C18 can also be used in NP and/or ANP when using higher percentages of organic mobile phase. The Diamond C18 is a cross between the Bidentate C18 and Diamond Hydride columns where the surface has both C18 moieties and shorter carbon chains. This column is still in the testing stages but is expected to exhibit similar abilities as the Bidentate C18, albeit slightly stronger effects on non-polar compounds. 
The third column, phenyl hydride, employs short chained phenyl groups which have high selectivity for aromatic compounds and those with high degrees of unsaturation. Finally, the Diol column consists of short chain diol groups which have higher selectivity for polar compounds and those susceptible to hydrogen bonding. None of the columns mentioned require end capping and are all compatible with mass spectrometry. Table 1 below shows the various column specifications including pore size/volume, column dimensions, $\mathrm{pH}$ ranges, maximum temperature, carbon loads and part/serial numbers.

Table 1. Column specifications. Includes column dimensions, particle dimensions, carbon load, $\mathrm{pH}$ ranges and maximum operating temperature.

\begin{tabular}{|c|c|c|c|c|c|}
\hline Bonded Phase & Pore Size & Surface Area & Pore Volume & Particle Size & Part Number \\
\hline \multirow{3}{*}{$\begin{array}{c}\text { Diamond } \\
\text { Bidentate C18 }\end{array}$} & $100 \AA$ & $390+/-30 \mathrm{~m} 2 / \mathrm{gm}$ & $1.00+/-0.10 \mathrm{~mL} / \mathrm{g}$ & $4 \mu \mathrm{m}$ & N/A - Beta Test \\
\hline & Dimensions & Carbon Load & pH Range & Max Temp & Serial Number \\
\hline & $4.6 \times 75 \mathrm{~mm}$ & $20.18 \%$ & $2.5-7.5$ & $80^{\circ} \mathrm{C}$ & C1388 \\
\hline \multirow{4}{*}{ Bidentate $\mathrm{C} 18^{\mathrm{rM}}$} & Pore Size & Surface Area & Pore Volume & Particle Size & Part Number \\
\hline & $100 \AA$ & $390+/-30 \mathrm{~m} 2 / \mathrm{gm}$ & $1.00+/-0.10 \mathrm{~mL} / \mathrm{g}$ & $4 \mu \mathrm{m}$ & 40018-75P \\
\hline & Dimensions & Carbon Load & pH Range & Max Temp & Serial Number \\
\hline & $4.6 \times 75 \mathrm{~mm}$ & $18-19 \%$ & $2.0-10.0$ & $80^{\circ} \mathrm{C}$ & $140408 \mathrm{~A}$ \\
\hline \multirow{4}{*}{ Diol } & Pore Size & Surface Area & Pore Volume & Particle Size & Part Number \\
\hline & $100 \AA$ & $390+/-30 \mathrm{~m} 2 / \mathrm{gm}$ & $1.00+/-0.10 \mathrm{~mL} / \mathrm{g}$ & $4 \mu \mathrm{m}$ & 40060-10P-2 \\
\hline & Dimensions & Carbon Load & pH Range & Max Temp & Serial Number \\
\hline & $2.1 \times 100 \mathrm{~mm}$ & $9-10 \%$ & $2.0-8.0$ & $80^{\circ} \mathrm{C}$ & C1516 \\
\hline \multirow{4}{*}{$\begin{array}{c}\text { Phenyl } \\
\text { Hydride }^{\mathrm{TM}}\end{array}$} & Pore Size & Surface Area & Pore Volume & Particle Size & Part Number \\
\hline & $100 \AA$ & $390+/-30 \mathrm{~m} 2 / \mathrm{gm}$ & $1.00+/-0.10 \mathrm{~mL} / \mathrm{g}$ & $4 \mu \mathrm{m}$ & $69020-10 P-2$ \\
\hline & Dimensions & Carbon Load & pH Range & Max Temp & Serial Number \\
\hline & $2.1 \times 100 \mathrm{~mm}$ & $10-12 \%$ & $2.0-8.0$ & $80^{\circ} \mathrm{C}$ & C1513 \\
\hline
\end{tabular}

\subsection{Instruments}

Three HPLC instruments used for this characterization project were a Perkin Elmer (PE) Flexar UPLC and two Agilent 1200 Series HPLC units. The PE instrument was equipped with both a UV-Vis detector and a single quadrupole MS detector (Figure 7). Only the UV-Vis detector was used for this project. The other components include solvent manager, autosampler (100 well plate), pumps/purging unit and Chromera 
software. The PE Flexar UPLC was used to collect acetonitrile data for all four columns. Software issues, instrumental failure and instrument location were detrimental for a portion of the study and will be discussed further in the following chapter.

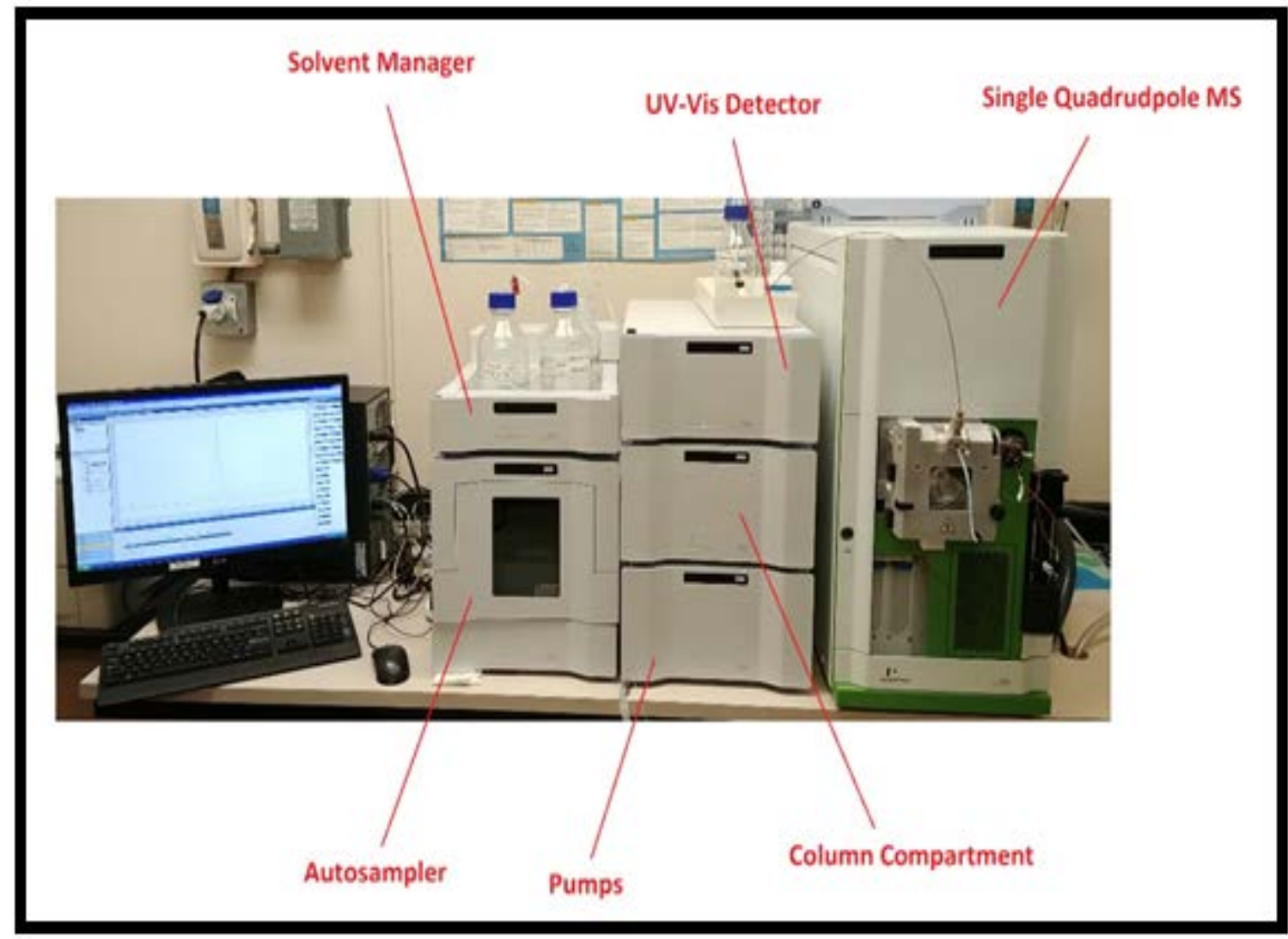

Figure 7. Perkin Elmer Flexar UPLC.

The Agilent 1200 Series HPLC units differed slightly in their respective components, but overall, instrumentation was identical (Figure 8). The first unit used is equipped with a G1322A Degasser, G1311A Quaternary Pump, G1315D DAD, G1316A Thermal Column Compartment (TCC), G1367B HiP-ALS and G1330B FC/ALS Thermostat. The second unit is equipped with a G1379B Degasser, G1312B Bin Pump SL, G1315C DAD SL, G1316B TCC SL, G1367D HiP-ALS SL + and G1330B FC/ALS Thermostat. Both instruments operate with ChemStation software. The main difference between the 
degassing units is the vacuum chamber volume in that the G1322A is $12 \mathrm{~mL}$ versus the G1379B is $0.5-1.0 \mathrm{~mL}$. The G1379B saves solvent by wasting less during routine flushing but has been associated with more failures than the former. ${ }^{34}$

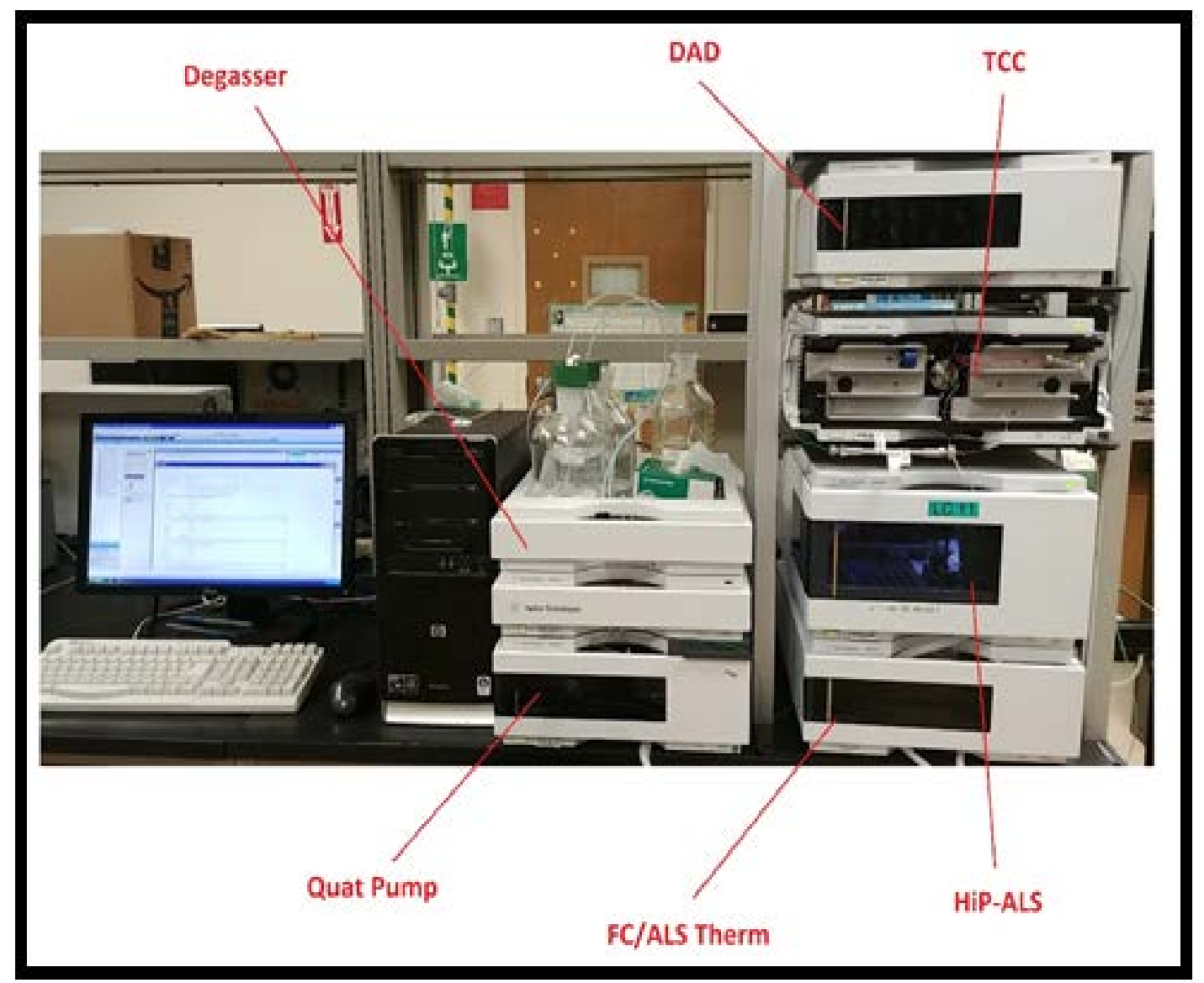

Figure 8. Agilent 1200 Series HPLC

The pumps differ slightly in that the quaternary is capable of mixing up to four solvents during a gradient while the binary is limited to two. In addition, the G1311A model's maximum operating pressure is 400 bar while the G1312B model can handle up to 600 bar, allowing for UPLC analysis. ${ }^{35}$ The detectors are identical in all aspects except that the G1315C can acquire data at a higher rate, $80 \mathrm{~Hz}$ compared to $20 \mathrm{~Hz}$. TCC units are identical but the G1316B delivers more stable temperatures and offers a maximum 
temperature of $100^{\circ} \mathrm{C}$. The difference between autosamplers is merely that the $\mathrm{D}$ model is compatible with a higher pressure (600 bar). Finally, the thermostats are identical for both instruments.

\subsection{Chemicals and Compounds}

\subsubsection{HPLC Solvents}

Methanol and acetonitrile were the main solvents used for the characterization of the four Type-C columns. Formic acid was used as buffer in both methanol and acetonitrile (0.1\%). HPLC to MS grade solvent was used, and the solvent is devoid of air, small particles and signal interferants. The solvents were acquired from Sigma-Aldrich, Omnisolv and Fisher Scientific manufacturers.

\subsubsection{Resveratrol Analogues}

Resveratrol and similar compounds were provided by Monash University in Melbourne, Australia. All compounds were of high purity, in powder form and delivered in small amber vials (1-2 mg of each compound). Each compound was dissolved in HPLC grade acetonitrile and vortexed/sonicated to give sample concentrations of 1 $\mathrm{mg} / \mathrm{mL}$. All standard samples of resveratrol and analogues were stored in amber vials and refrigerated at $-4{ }^{\circ} \mathrm{C}$. Injected samples were diluted 10 -fold using $100 \mathrm{uL}$ polypropylene vial inserts at a final concentration of $0.1 \mathrm{mg} / \mathrm{mL}$. Figure 9 below shows the structure of each compound and their respective IUPAC names. All compounds were labeled from MH 1 to MH 23 and will be referred to as such throughout this paper. 


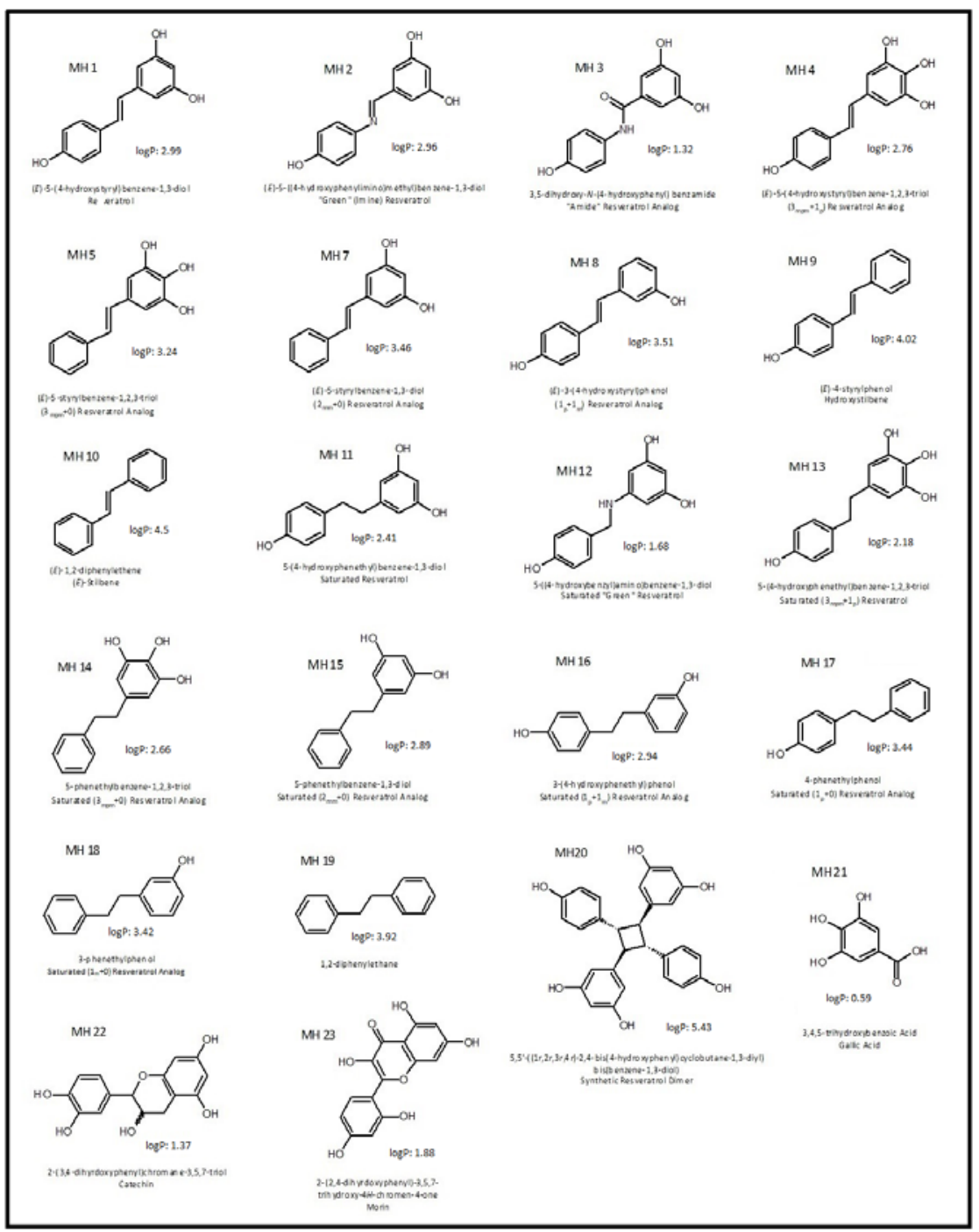

Figure 9. Resveratrol Analogues. Depicts the structures, IUPAC names and partition coefficients of the various resveratrol compounds. (ChemSketch Software and Molinspiration Property Engine)

\subsubsection{Acidic, Basic and Neutral Compounds}

The acidic, basic and neutral compounds were acquired from Sigma-Aldrich in high purity. Two acidic compounds were used, ketoprofen and 2-nitrobenzoic acid.

Ketoprofen is a common non-steroidal anti-inflammatory drug while 2-nitrobenzoic acid is an intermediate used to synthesize a variety of chemicals. The basic compounds analyzed were amitriptyline and diphenhydramine. Amitriptyline is an 
antipsychotic/antidepressant drug while diphenhydramine is a widely used sleep aid and allergy medicine. The last compounds of this set are neutral: anisole, naphthalene and ethylbenzene. All three are aromatic and serve as synthetic intermediates in a variety of reactions. Figure 8 below shows the structures of the acidic, basic and neutral compounds mentioned above.

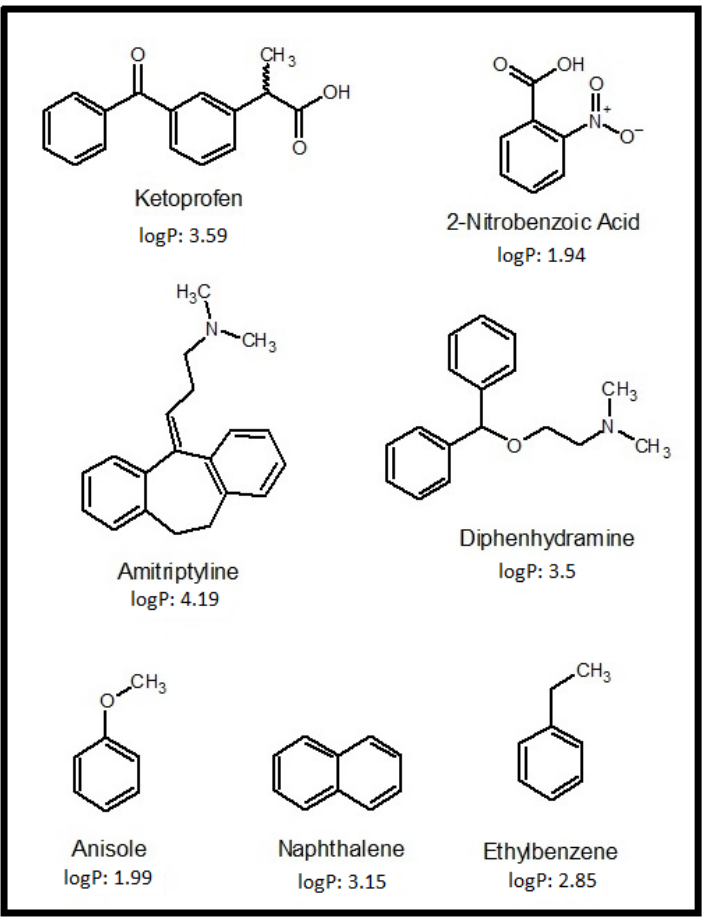

Figure 10. Acidic, Basic and Neutral Compounds. Depicts the structure, name and partition coefficient of each compound. (ChemSketch Software and Molinspiration Property Engine)

\subsection{Methods}

All methods conducted for this project were carried out in the isocratic mode, where the mobile phase composition and flow rate remained constant throughout each run. Each set of standard samples were subjected to the same methods where the initial mobile phase concentration was $90 \%$ organic mobile phase (acetonitrile or methanol) and $10 \%$ deionized water. The flow rate was maintained at $0.4 \mathrm{~mL} / \mathrm{min}$ and the injection volume 
was set to $1 \mathrm{uL}$. Subsequent analyses demanded that the mobile phase composition be reduced by $10 \%$ resulting in a range from $90 \%$ organic to $10 \%$ organic. Wavelengths observed are depicted below in Table 2.

Table 2. Wavelength Parameter. Wavelengths were chosen based on literary values and collaborator suggestions. The switch to the Agilent 1200 Series instruments allowed for a more thorough investigation of optimal wavelengths.

\begin{tabular}{|c|c|c|}
\hline Instrument & $\begin{array}{c}\text { Resveratrol } \\
\text { Analogues }\end{array}$ & $\begin{array}{c}\text { Acidic/Basic/Neutral } \\
\text { Compounds }\end{array}$ \\
\hline $\begin{array}{c}\text { PE UPLC (UV- } \\
\text { Vis) }\end{array}$ & $\begin{array}{c}254 \text { and } 308 \\
\mathrm{~nm}\end{array}$ & N/A \\
\hline $\begin{array}{c}\text { Agilent } 1200 \\
\text { (DAD) } 1\end{array}$ & $\begin{array}{c}220,254,280, \\
308 \text { and 335 nm }\end{array}$ & $254 \mathrm{~nm}$ \\
\hline & & $254 \mathrm{~nm}$ \\
\hline $\begin{array}{c}\text { Agilent } 1200 \\
\text { (DAD) } 2\end{array}$ & $\begin{array}{r}220,254,280, \\
\text { (D) }\end{array}$
\end{tabular}




\section{Chapter 3: Results and Discussion}

For the purpose of characterizing the four Type-C silica columns, two sets of compounds were utilized, resveratrol analogues and acidic/basic/neutral compounds. The resveratrol analogues are used to observe ANP/RP retention and to determine separation capabilities while the acidic/basic/neutral set is used to characterize the columns based on specific molecular interactions. Both groups were analyzed using acetonitrile and methanol mobile phases (both with $0.1 \%$ formic acid), ranging from $90 \%$ organic to $10 \%$ organic. Samples were prepared between $0.1-0.5 \mathrm{mg} / \mathrm{mL}$ prior to injection and were run in triplicate. Tabulated data depict the averaged retention times of each compound and specify the stationary phase used for the corresponding mobile phase compositions. The

first set of figures are retention plots showing the varying retention times (y-axis) of each compound from 0 - 45 minutes across the mobile phase spectrum (x-axis). Data collection was halted for compounds that were retained longer than 45 minutes or if peak shape was too broad or poor. The second set of figures show stacked and overlaid chromatograms of the compounds of interest. The chromatograms are separated between resveratrol compounds and the acidic/basic/neutral set. The tables of data give precise retention averages while the retention maps give visual understanding to retention trends between the two groups of compounds. Finally, the stacked overlays show the theoretical trends of resolution if the components were introduced as a mixture. All data collected and analyzed were based solely on qualitative information.

The PE UPLC equipped with a UV-Vis detector was used initially for the analysis of resveratrol compounds with acetonitrile as the mobile phase. Samples weighing between 
$1.0 \mathrm{mg}$ to $2.8 \mathrm{mg}$ were delivered to SJSU from Melbourne, Australia. Stock standards were initially prepared in amber vials, in minimal light, at concentrations between 1.0 $1.4 \mathrm{mg} / \mathrm{mL}$ using acetonitrile as diluent. A tenfold dilution was performed to give standard samples concentrations ranging from $0.1-0.4 \mathrm{mg} / \mathrm{mL}$ prior to injection. Methods and sequences prepared on Chromera software follow the previously mentioned parameters. At first, all compounds were observed at $308 \mathrm{~nm}$ (recommended by Monash University and comparable to literature wavelengths). ${ }^{36}$ Little to no signal was observed at $308 \mathrm{~nm}$ for MH 11-19 and 22. Due to working with small amounts of sample, a UV-vis spectrometer was not used to determine the optimal wavelength for these compounds. The wavelength of $254 \mathrm{~nm}$ was tested for the aforementioned compounds and was determined to give sufficient signal for qualitative data. Although the PE UPLC was equipped with a temperature controlled autosampler (including insulated sample tray cover), the temperature in the room could not be controlled well. Because of this, the evaporation of solvent within the HPLC vials was inevitable. Constant refilling of solvent and re-spiking sample vials significantly affected the concentration of each sample, resulting in variation of absorbance.

According to the structures and partition coefficients shown in Figure 7, the unsaturated resveratrol compounds (MH1, 2, 4 - 10) should exude higher retention than the saturated versions (MH 3, 11-19) for the RP columns, Diamond Bidentate C18 and Bidentate C18. The phenyl hydride should behave similarly to the previous RP columns with additional pi stacking interactions. Finally, the diol column is expected to exhibit ANP retention with more polar compounds. Considering that the resveratrol analogues 
are considered nonpolar, with the exception of MH 21 (gallic acid), minimal retention is assumed. Data were compared to the $\mathrm{KNO}_{3}$ dead time and Table 3 gives the expected trends. Comparisons between the actual and expected trends will be discussed later. The first and fourth trends are based on the transition from resveratrol to an imine/amine (MH2/MH12) analogue and finally to an amide (MH3) version. The second and sixth trends show the subsequent additions of hydroxide groups to stilbene (MH10) and diphenyl ethane (MH19). Trends \#3 and \#5 compare the differences between having a diol plus an unsubstituted benzene or two benzenes with single hydroxide attachment. The seventh trend evaluates the effect of meta versus para position hydroxide place. Finally, trend \#8 compares resveratrol dimer, two flavonoids (MH22 and MH23) and gallic acid.

Table 3. Retention trends of resveratrol and analogues based on structure and polarity.

\begin{tabular}{|c|c|c|}
\hline Trend \# & Reversed Phase & Aqueous Normal Phase \\
\hline 1 & MH1 $>$ MH2 $>$ MH3 & MH3 $>$ MH2 $>$ MH 1 \\
\hline 2 & MH11 $>$ MH12 $>$ MH3 & MH4 $>$ MH1 $>$ MH5 $>$ MH7 $>$ MH9 $>$ MH10 \\
\hline 3 & MH8 $>$ MH7 & MH7 $>$ MH8 \\
\hline 4 & MH16 $>$ MH15 & MH3 $>$ MH12 $>$ MH11 \\
\hline 5 & MH17 $>$ MH18 & MH15 $>$ MH16 \\
\hline 6 & MH10 $>$ MH9 $>$ MH7 $>$ MH5 $>$ MH1 $>$ MH4 & MH13 $>$ MH11 $>$ MH14 $>$ MH15 $>$ MH17 $>$ MH19 \\
\hline 7 & MH19 $>$ MH17 $>$ MH15 $>$ MH14 $>$ MH11 $>$ MH13 & MH18 $>$ M17 \\
\hline 8 & MH20 $>$ MH1 $>$ MH23 $>$ MH22 $>$ MH21 & MH21 $>$ MH22 $>$ MH23 $>$ MH1 $>$ MH20 \\
\hline
\end{tabular}

Since the trends mentioned above only account for polarity, it is important to account for other analyte to stationary phase interactions. The linear solvation energy relationship (LSER) equation shown below (Eq. 11) gives several possible interactions other than hydrophobicity that may explain why the expected trends are not absolute.

Eq. $11 \log k=\log k_{0}+\eta^{\prime} H+\sigma^{\prime} S+\beta^{\prime} A+\alpha^{\prime} B+\kappa^{\prime} C$ 
The LSER equation relates retention factor $k$ of various compounds with several parameters and descriptors associated with the stationary phase. ${ }^{37}$ Parameters $H, S, A, B$ and $C$ correspond to hydrophobicity, steric effects, hydrogen bond acidity/basicity and electrostatic interactions. ${ }^{38}$ For the scope of this work, these parameters and descriptors will not be discussed in great detail. However, it is important to account for these potential interactions when comparing different stationary phases using the same set of analytes and mobile phase compositions. ${ }^{39}$ Collaborators from Monash University will be utilizing the data collected from this project and will calculate these parameters for more precise characterization.

\subsection{Data with Acetonitrile as Mobile Phase}

\subsubsection{Resveratrol Analogues}

From the Diamond Bidentate C18 data shown in Table 4 and Figure 11, the typical RP trend was observed. As acetonitrile was decreased from $90 \%$ to $30 \%$ compositions, retention of all resveratrol analogues was increased. In comparison to $\mathrm{KNO}_{3}$ dead time, significant retention $(k>2)$ was seen for 13 of the 22 compounds while the remainder showed minimal retention $(k<2)$. When addressing the trends in Table 3, only Trend 1,2 and 6 were coherent with the expected retention order. 
Table 4. Diamond Bidentate C18 Capacity Factor " $k$ " (ACN/Resveratrol). Average of in triplicate injections from $30 \%$ organic to $90 \%$ organic.

\begin{tabular}{|c|c|c|c|c|c|c|c|c|}
\hline & \multicolumn{8}{|c|}{ Diamond Bidentate C18 } \\
\hline & \multirow{2}{*}{ Resveratrol Analogues } & \multicolumn{7}{|c|}{ Mobile Phase Composition (\% Acetonitrile) } \\
\hline & & 30 & 40 & 50 & 60 & 70 & 80 & 90 \\
\hline \multirow{22}{*}{ 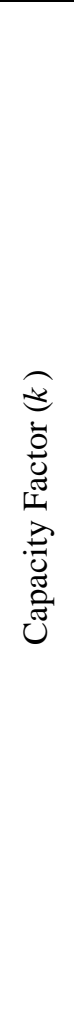 } & MH 1 & 2.92 & 0.71 & 0.25 & 0.1 & 0.02 & -0.02 & -0.06 \\
\hline & MH 2 & 0.54 & 0.21 & 0.1 & 0.05 & 0.01 & 0 & -0.03 \\
\hline & MH 3 & 0.45 & 0.1 & 0.01 & -0.02 & -0.05 & -0.05 & -0.08 \\
\hline & MH 4 & 1.58 & 0.4 & 0.14 & 0.04 & -0.01 & -0.03 & -0.07 \\
\hline & MH 5 & 13.29 & 2.28 & 0.84 & 0.42 & 0.21 & 0.1 & 0.03 \\
\hline & MH 7 & & 3.8 & 1.3 & 0.62 & 0.3 & 0.15 & 0.06 \\
\hline & MH 8 & & 2.76 & 0.97 & 0.45 & 0.21 & 0.1 & 0.02 \\
\hline & MH 9 & & 15.73 & 4.82 & 2.16 & 1.08 & 0.59 & 0.29 \\
\hline & MH 10 & & & 24.67 & 10.07 & 4.69 & 2.41 & 1.1 \\
\hline & MH 11 & & 0.74 & 0.29 & 0.11 & 0.04 & -0.01 & -0.03 \\
\hline & MH 12 & 0.54 & 0.37 & 0.27 & 0.2 & 0.27 & 0.15 & 0.14 \\
\hline & MH 13 & 0.52 & 1.29 & 0.41 & 0.15 & 0.02 & -0.02 & -0.05 \\
\hline & MH 14 & & 15.56 & 4.03 & 1.54 & 0.65 & 0.12 & 0.04 \\
\hline & MH 15 & 14.25 & 4.1 & 1.67 & 0.86 & 0.52 & 0.34 & 0.24 \\
\hline & MH 16 & 10.51 & 3.23 & 1.38 & 0.73 & 0.44 & 0.3 & 0.21 \\
\hline & MH 17 & & 15.5 & 4.94 & 2.22 & 1.11 & 0.6 & 0.3 \\
\hline & MH 18 & & 16.03 & 4.97 & 2.23 & 1.12 & 0.59 & 0.29 \\
\hline & MH 19 & & & 25.85 & 10.28 & 4.76 & 2.39 & 1.09 \\
\hline & MH 20 & 1.84 & 0.61 & 0.29 & 0.17 & 0.09 & 0.07 & 0.06 \\
\hline & MH 21 & 0.11 & 0.12 & 0.08 & 0.07 & 0.07 & 0.08 & 0.08 \\
\hline & MH 22 & 0.25 & 0.17 & 0.1 & 0.08 & 0.07 & 0.07 & 0.07 \\
\hline & MH 23 & 6.3 & 0.62 & 0.24 & 0.1 & 0.02 & -0.02 & -0.05 \\
\hline
\end{tabular}

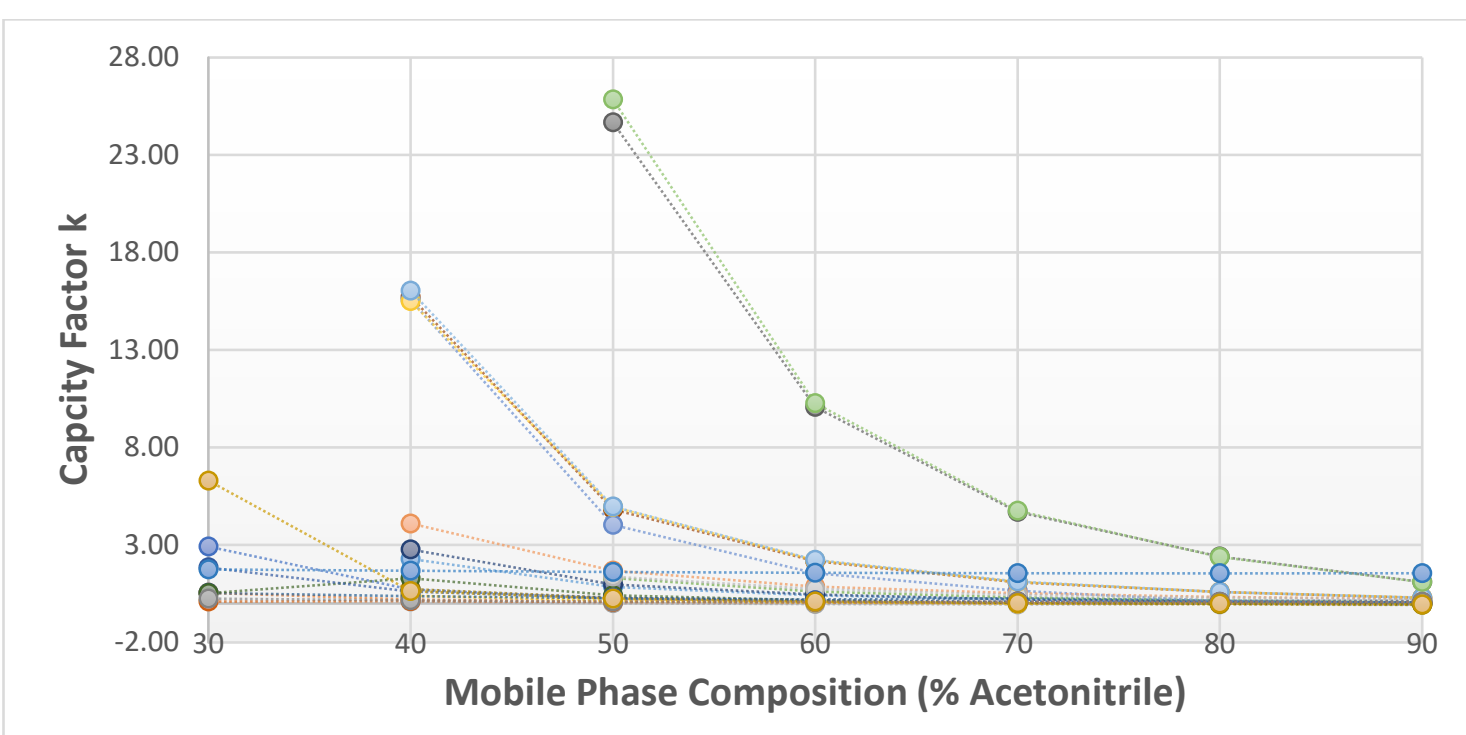

Figure 11. Diamond C18 Retention Map (ACN/Resveratrol). Compares capacity factor "k" (y-axis) to percentage acetonitrile mobile phase (x-axis). 
MH8 has a slightly higher logP and differs from MH7 by having a single hydroxide group on each benzene while MH7 contains a diol and a bare benzene ring. The structure of MH7 is surfactant-like containing a hydrophobic tail (unsubstituted benzene) and hydrophilic head (diol substituted) which may explain the difference in elution order with MH8. When comparing MH16 to MH15 a similar conclusion is reached explaining the higher retention for MH15 (surfactant structure). The higher retention that MH14 and MH13 display over MH15 and MH11 is unclear but may be associated with more polar heads forcing interaction with the less substituted benzene tail. The difference in polarity of MH17 and MH18, meta vs. para hydroxide isomers, was negligible and the order of retention not stressed upon. Complete separation of these structural isomers was not observed but the DC18 column gave the second highest selectivity for them. Finally, MH23 (logP = 1.88) exhibited significantly higher retention than MH20 (logP = 5.43) despite being more polar. This may be due to steric hinderance associated with MH20's bulkiness. The overlaid/stacked chromatograms for the Diamond Bidentate C18 using the PE UPLC and acetonitrile as mobile phase (Figure 12) showed that few compounds have high enough selectivity to be fully resolved from one another. Even at $40 \%$ organic the majority of compounds overlap from 2 - 10 minutes. Despite having unique retention times, if introduced as a mixture, this particular set of compounds would be difficult to separate using Diamond Bidentate C18 stationary and acetonitrile mobile phase. Potential solutions would be gradient method development and reduced standard/sample concentrations. 


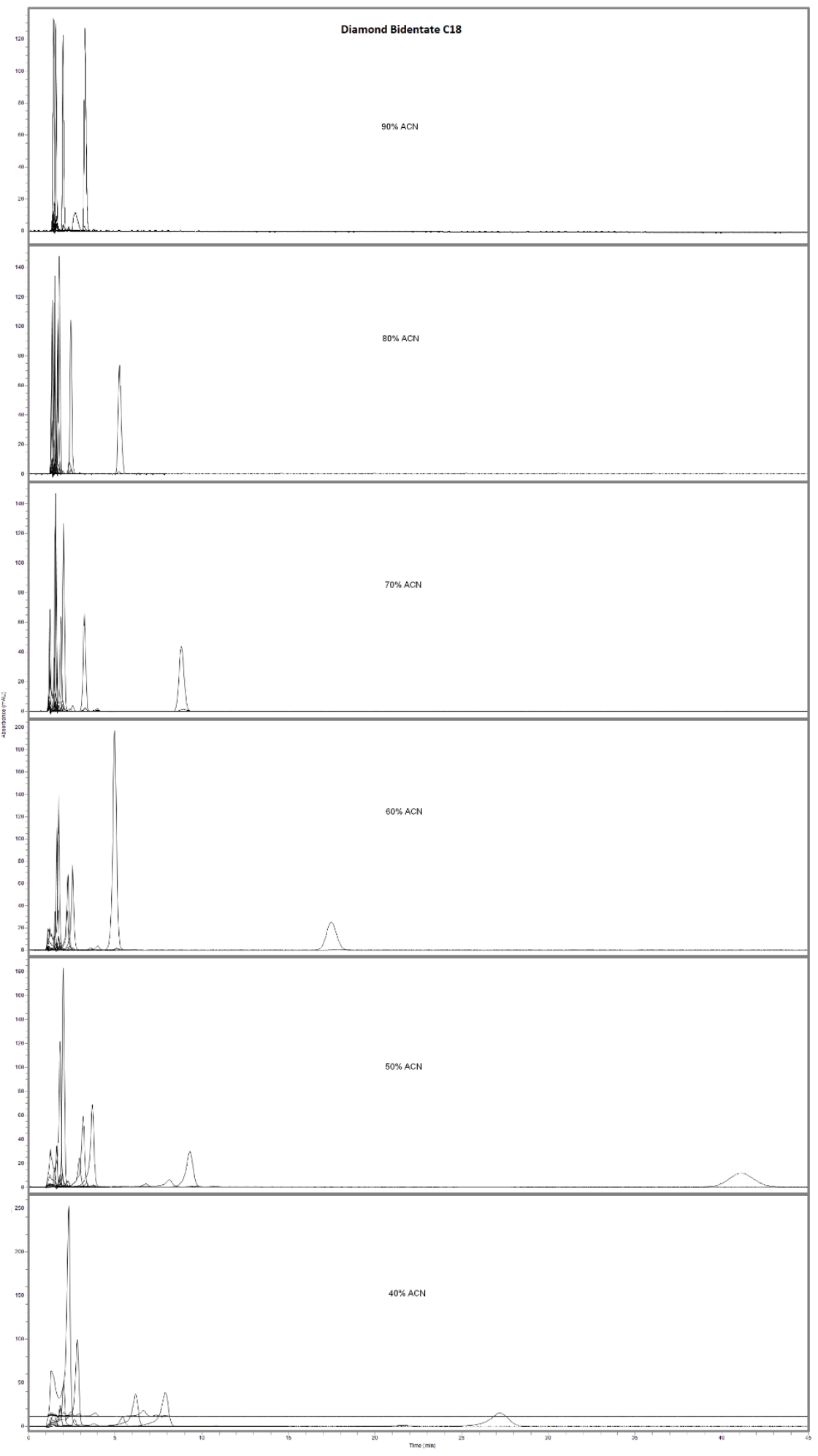

Figure 12. Diamond Bidentate C18 with Acetonitrile Mobile Phase Overlaid/Stacked Chromatograms (Resveratrol). 
Similarly, to the Diamond C18, the Bidentate C18 shows the same RP trend as organic mobile phase is reduced from 90\% - 10\% (Figure 13). When comparing retention data between the two, the Bidentate C18 only displayed significant retention (at 30\% acetonitrile) for 11 compounds compared to 13 on the Diamond C18 (Table 5). This information supports that the Diamond C18 is more powerful in retaining this set of nonpolar compounds when using acetonitrile as mobile phase. When referring to the trends in Table 3, the Bidentate C18 demonstrated comparable retention capabilities. The same issues were seen for Trends 3, 5, 6, 7 and 8. In addition, the retention times for MH17, 18 and 19 were questionably low in contrast to the Diamond C18. At this time, the cause of this variance remains unclear. Figure 14 shows the overlaid/stacked chromatograms and gives a similar assessment where the overlap observed would make complete resolution difficult.

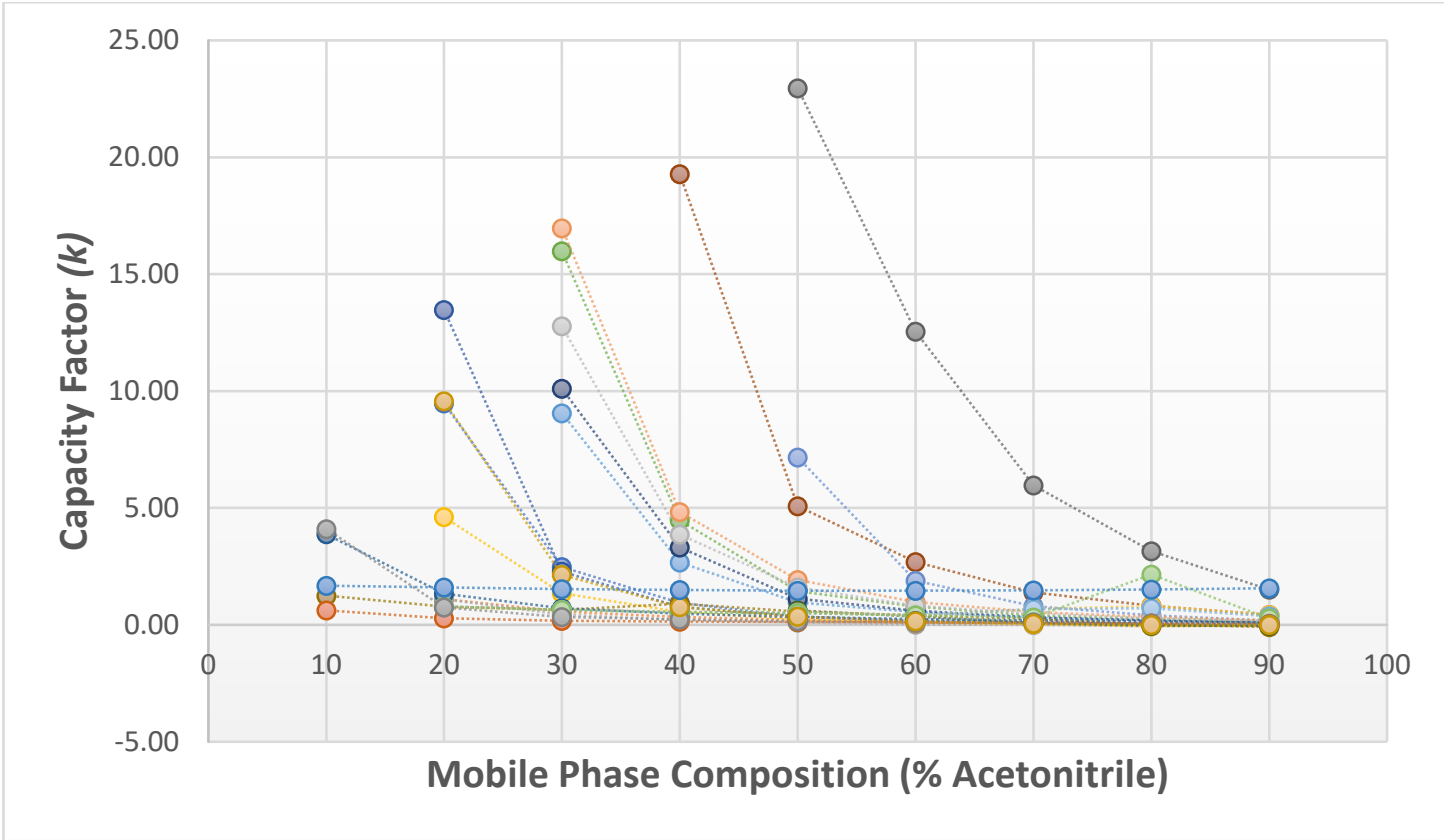

Figure 13. Bidentate C18 Retention Map (ACN/Resveratrol). Compares capacity factor " $k$ " (y-axis) to percentage acetonitrile mobile phase (x-axis). 
Table 5. Bidentate C18 Capacity Factor " $k$ ” (ACN/Resveratrol). Average of in triplicate injections from $10 \%$ organic to $90 \%$ organic.

\begin{tabular}{|c|c|c|c|c|c|c|c|c|}
\hline & \multicolumn{8}{|c|}{ Bidentate C18 } \\
\hline & \multirow{2}{*}{ Resveratrol Analogues } & \multicolumn{7}{|c|}{ Mobile Phase Composition (\% Acetonitrile) } \\
\hline & & 10 & 20 & 30 & 40 & 50 & 60 & 70 \\
\hline \multirow{22}{*}{ 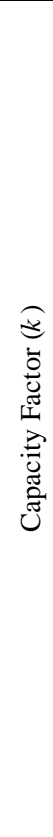 } & MH 1 & & 9.47 & 2.48 & 0.94 & 0.36 & 0.17 & 0.08 \\
\hline & MH 2 & & 1.1 & 0.56 & 0.33 & 0.21 & 0.11 & 0.06 \\
\hline & MH 3 & & 1.1 & 0.44 & 0.2 & 0.11 & 0.02 & 0 \\
\hline & MH 4 & & 4.61 & 1.35 & 0.55 & 0.24 & 0.11 & 0.04 \\
\hline & MH 5 & & & 9.05 & 2.67 & 0.96 & 0.54 & 0.31 \\
\hline & MH 7 & & & 15.98 & 4.47 & 1.44 & 0.78 & 0.42 \\
\hline & MH 8 & & & 10.1 & 3.3 & 1.13 & 0.61 & 0.32 \\
\hline & MH 9 & & & & 19.27 & 5.08 & 2.69 & 1.39 \\
\hline & MH 10 & & & & & 22.96 & 12.54 & 5.98 \\
\hline & MH 11 & 1.25 & 0.78 & 0.63 & 0.91 & 0.57 & 0.4 & 0.26 \\
\hline & MH 12 & 3.88 & 1.34 & 0.72 & 0.48 & 0.33 & 0.25 & 0.19 \\
\hline & MH 13 & & & & & 0.62 & 0.35 & 0.08 \\
\hline & MH 14 & & & & & 7.17 & 1.89 & 0.81 \\
\hline & MH 15 & & & 16.97 & 4.84 & 1.93 & 0.97 & 0.55 \\
\hline & MH 16 & & & 12.77 & 3.86 & 1.61 & 0.82 & 0.48 \\
\hline & MH 17 & & 0.77 & 0.64 & 0.56 & 0.53 & 0.4 & 0.67 \\
\hline & MH 18 & & 0.79 & 0.64 & 0.55 & 0.51 & 0.4 & 0.67 \\
\hline & MH 19 & & 0.78 & 0.64 & 0.54 & 0.5 & 0.4 & 0.32 \\
\hline & MH 20 & & 13.47 & 2.29 & 0.77 & 0.35 & 0.18 & 0.1 \\
\hline & MH 21 & 0.62 & 0.28 & 0.18 & 0.15 & 0.13 & 0.1 & 0.08 \\
\hline & MH 22 & 4.11 & 0.74 & 0.35 & 0.23 & 0.15 & 0.11 & 0.08 \\
\hline & MH 23 & & 9.57 & 2.14 & 0.76 & 0.36 & 0.15 & 0.06 \\
\hline
\end{tabular}

Table 6. Phenyl Hydride Capacity Factor " $k$ " (ACN/Resveratrol). Average of in triplicate injections from $10 \%$ organic to $90 \%$ organic.

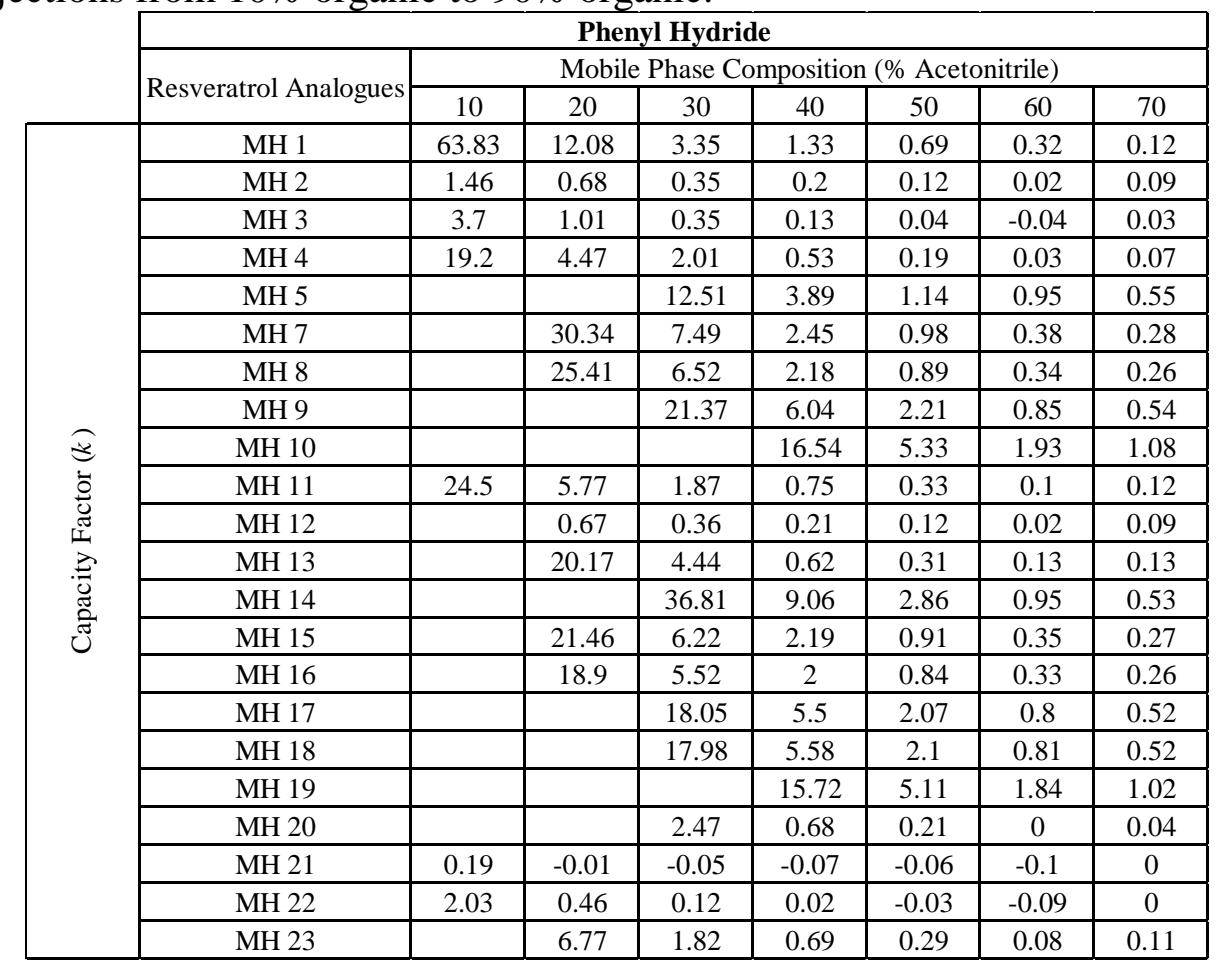




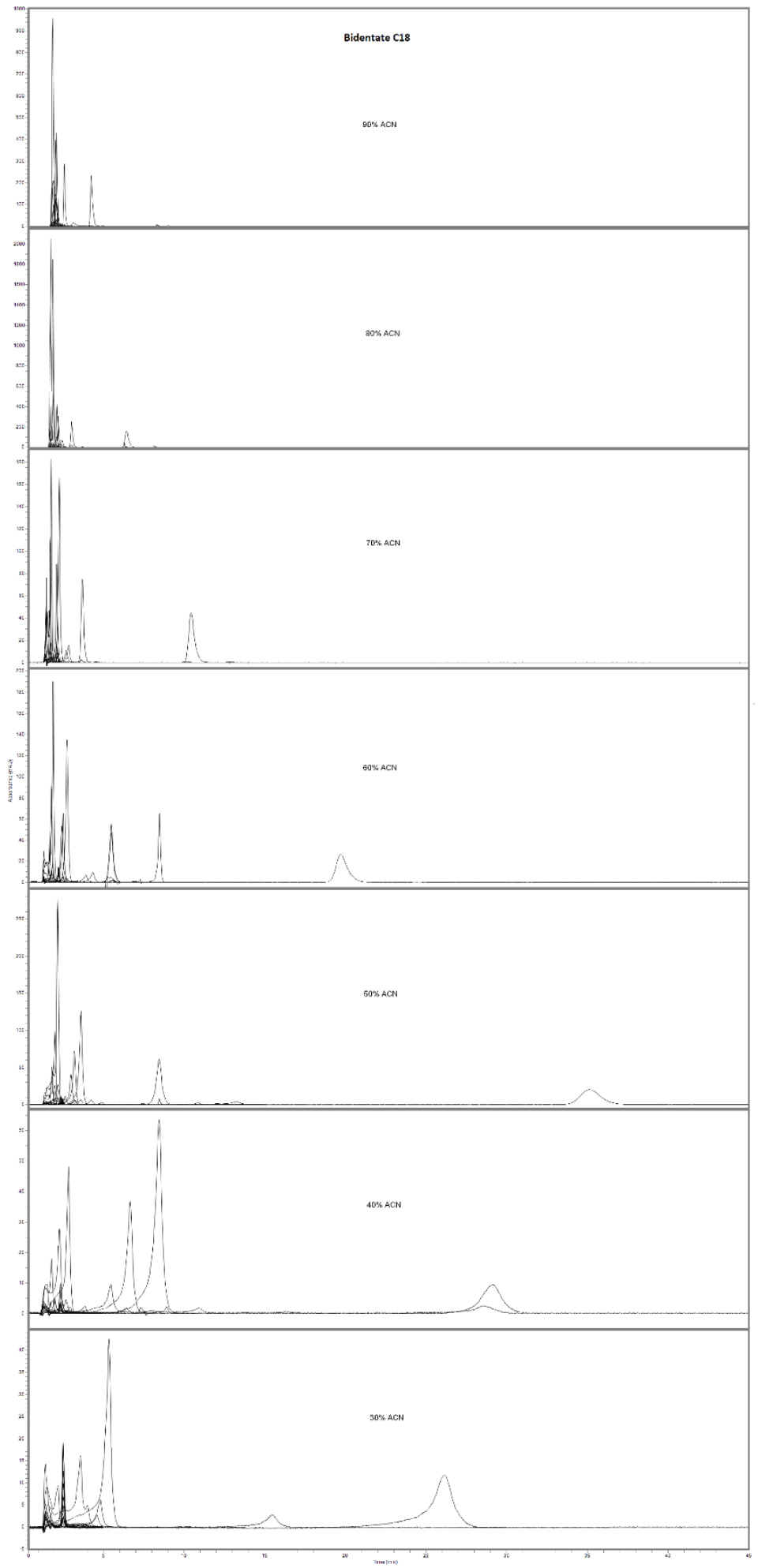

Figure 14. Bidentate C18 with Acetonitrile Mobile Phase Overlaid/Stacked Chromatograms (Resveratrol). 
The Phenyl Hydride data shown was collected from the Agilent 1200 Series (1). The dimensions of the Phenyl Hydride and the Diol were 2.1 x 100mm compared to 4.6 x $75 \mathrm{~mm}$ for the Diamond C18 and Bidentate C18. Since the flow rates $(0.4 \mathrm{~mL} / \mathrm{min})$ and injection volumes ( $1 \mathrm{uL}$ ) were maintained throughout the study, the capacity factor $k$ of the Phenyl Hydride and Diol columns can be theoretically compared to the Diamond C18 and Bidentate C18 columns. Similar to the Diamond C18 and Bidentate C18 columns, the Phenyl Hydride operates in a RP manner where retention is increased as percent organic is decreased (Table 6). Of the unsaturated resveratrol analogues, MH4 and MH5 were the only ones that gave less retention than their respective saturated versions (MH13 and MH14). Unfortunately, no explanation for this incident has been determined. In reference to the polarity tendencies, only Trends 7 and 8 were completely agreeable. In Trends 1 and 4, MH3 exhibited higher retention than MH2/MH12 despite having a low logP value. This phenomenon could be attributed to the central keto group on MH3 but the associated interactions are unclear.

In Trends 2, 3, 5 and 6 the surfactant-like properties of MH5, 7, 14 and 15 overshadowed the expected retention scheme. Figure 15 shows that significant retention $(k>2)$ is observed for 19 of 22 resveratrol analogues. Figure 16 displays the progression of separation across all mobile phase compositions. Significant selectivity is observed for the majority of the compounds where the most difficult to separate were MH2/MH12 and MH17/MH18. Overall, the Phenyl Hydride shows the highest potential in separating this set of resveratrol compounds in acetonitrile. Furthermore, considering that full resolution 
is achieved $(\approx 1 \mathrm{mg} / \mathrm{mL})$ at $10 \%$ acetonitrile, analysts can save on solvent expenses and utilize gradient methods to optimize separations.

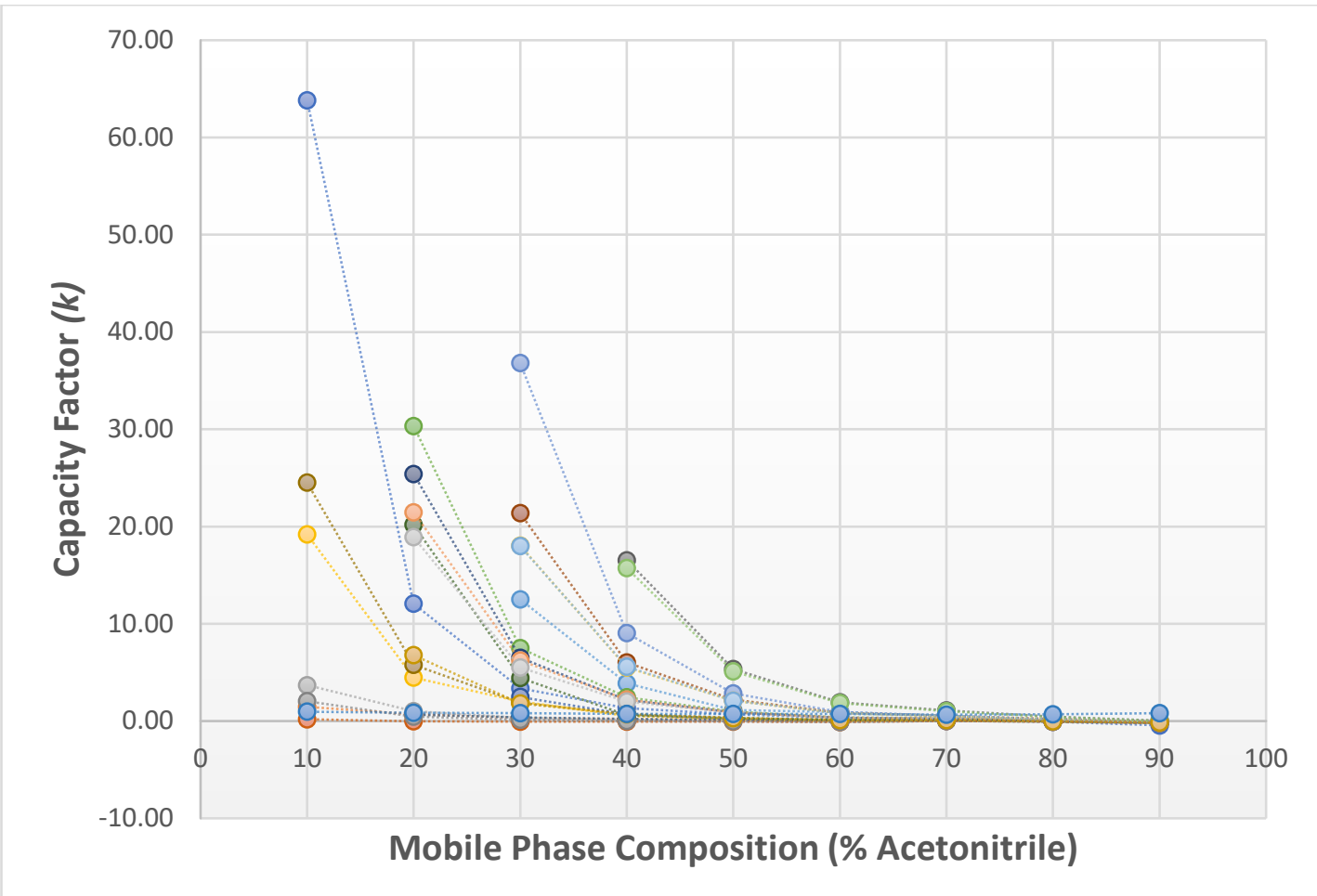

Figure 15. Phenyl Hydride Retention Map (ACN/Resveratrol). Compares capacity factor “ $k$ ” (y-axis) to percentage acetonitrile mobile phase (x-axis).

The Diol data shown was collected from the Agilent 1200 Series (2). Compared to the previous columns, the Diol is considered to be an ANP column. The resveratrol compounds are considerably nonpolar and are expected to exhibit minimal retention with Diol stationary phase. Indeed, from data shown in Table 7 and Figure 17, the majority of the compounds (except MH9 and MH10) display little to no retention across the mobile phase spectrum. Although only slightly, the Diol was the sole column that demonstrated dual retention (ANP: 60-90\%B, RP: 50-10\%B) with MH2 (imine). Referencing the expected polarity trends is more complex due to the presence of two modes of retention. 


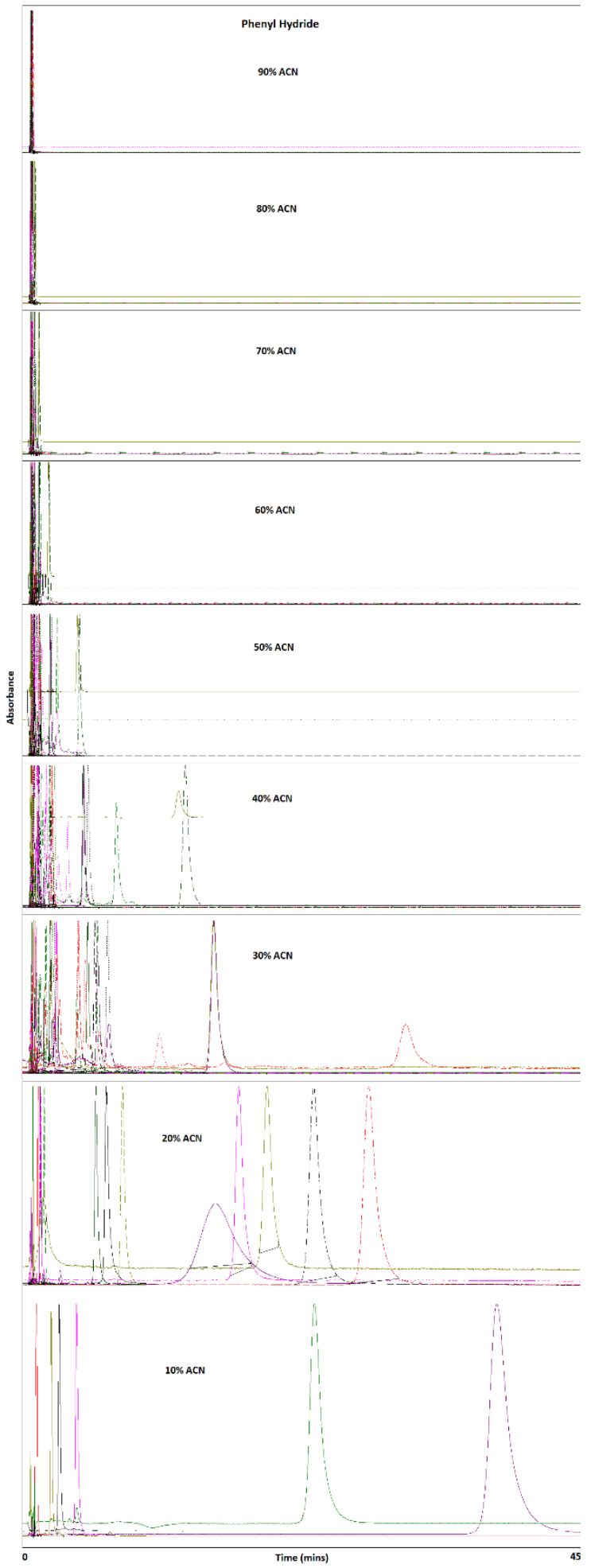

Figure 16. Phenyl Hydride with Acetonitrile Mobile Phase Overlaid/Stacked Chromatograms. 
Table 7. Diol Capacity Factor " $k$ " (ACN/Resveratrol). Average of in triplicate injections from $10 \%$ organic to $90 \%$ organic.

\begin{tabular}{|c|c|c|c|c|c|c|c|c|}
\hline & \multicolumn{8}{|c|}{ Diol } \\
\hline & \multirow{2}{*}{ Resveratrol Analogues } & \multicolumn{7}{|c|}{ Mobile Phase Composition (\% Acetonitrile) } \\
\hline & & 10 & 20 & 30 & 40 & 50 & 60 & 70 \\
\hline \multirow{22}{*}{ 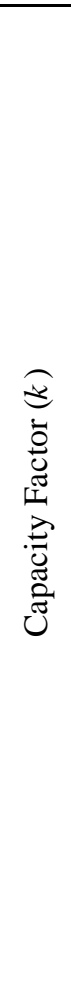 } & MH 1 & 1.12 & 0.31 & 0.13 & 0.07 & 0.01 & -0.05 & $\begin{array}{c}-0.12 \\
\end{array}$ \\
\hline & MH 2 & 0.2 & 0.15 & 0.13 & 0.1 & 0.18 & 0.24 & 0.32 \\
\hline & MH 3 & 0.28 & 0.16 & 0.11 & 0.08 & 0.04 & -0.01 & -0.08 \\
\hline & MH 4 & 0.44 & 0.3 & 0.15 & 0.08 & 0.02 & -0.04 & -0.11 \\
\hline & MH 5 & 1.35 & 0.46 & 0.2 & 0.07 & 0 & -0.06 & -0.12 \\
\hline & MH 7 & 2.67 & 0.57 & 0.19 & 0.07 & 0 & -0.06 & -0.13 \\
\hline & MH 8 & 2.21 & 0.48 & 0.17 & 0.07 & 0 & -0.06 & -0.14 \\
\hline & MH 9 & 7.48 & 1.28 & 0.3 & 0.09 & 0 & -0.07 & -0.15 \\
\hline & MH 10 & 30.12 & 4.63 & 0.8 & 0.19 & 0.02 & -0.07 & -0.15 \\
\hline & MH 11 & 0.5 & 0.2 & 0.11 & 0.06 & 0.01 & -0.05 & -0.12 \\
\hline & MH 12 & 0.2 & 0.16 & 0.13 & 0.1 & 0.06 & -0.01 & -0.08 \\
\hline & MH 13 & 0.26 & 0.19 & 0.11 & 0.06 & 0 & -0.05 & -0.11 \\
\hline & MH 14 & 0.25 & 0.21 & 0.2 & 0.07 & $\begin{array}{l}-0.01 \\
\end{array}$ & -0.07 & -0.13 \\
\hline & MH 15 & 1.41 & 0.36 & 0.15 & 0.07 & 0 & -0.06 & -0.13 \\
\hline & MH 16 & 1.21 & 0.32 & 0.14 & 0.06 & 0 & -0.06 & -0.13 \\
\hline & MH 17 & 4.68 & 0.91 & 0.24 & 0.08 & 0 & $\begin{array}{l}-0.07 \\
\end{array}$ & -0.15 \\
\hline & MH 18 & 4.62 & 0.9 & 0.24 & 0.08 & 0 & -0.07 & -0.15 \\
\hline & MH 19 & & 4.06 & 0.72 & 0.17 & 0.01 & -0.07 & -0.15 \\
\hline & MH 20 & 4.13 & 0.38 & 0.1 & 0.04 & -0.01 & -0.06 & -0.13 \\
\hline & MH 21 & 0.16 & 0.13 & 0.12 & 0.1 & 0.07 & 0.04 & -0.02 \\
\hline & MH 22 & 0.22 & 0.15 & 0.11 & 0.08 & 0.05 & 0.01 & -0.05 \\
\hline & MH 23 & 1.21 & 0.32 & 0.14 & 0.08 & 0.02 & -0.04 & -0.11 \\
\hline
\end{tabular}

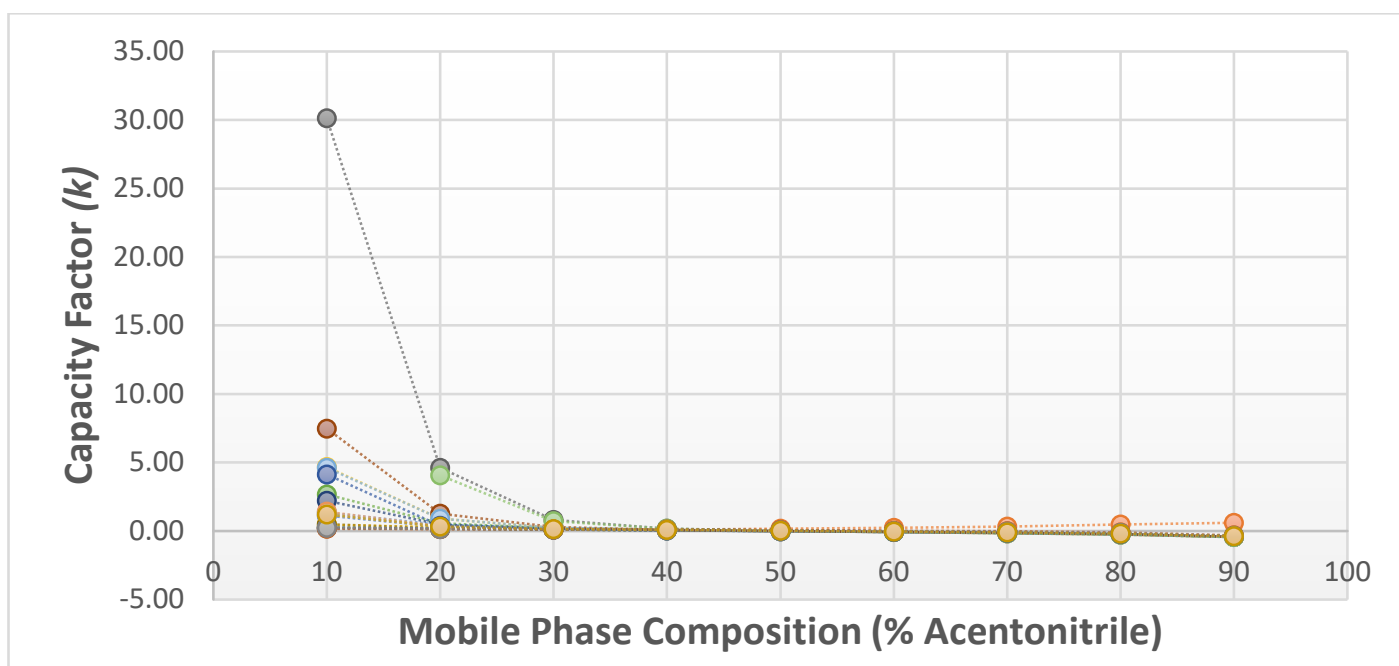

Figure 17. Diol Retention Map (ACN/Resveratrol). Compares capacity factor “ $k$ ” ( $y$ axis) to percentage acetonitrile mobile phase (x-axis). 
Trends 2, 4, 6 and 8 were compliant for the ANP portion and Trends 2, 6, 7 and 8 for the RP portion. When operating in ANP, MH7/MH8 and MH15/MH16 were inseparable but in RP mode the surfactant displayed similar retention patterns as the aforementioned columns. The "amide resveratrol” (MH3) still exhibited higher retention than the imine (MH2) and amine (MH12) versions. This shows that MH3 is selectively retained on the Phenyl and Diol columns compared to the Diamond and Bidentate C18 stationary phases when using acetonitrile as mobile phase. From overlaid and stacked chromatograms (Figure 18), the unique dual retention is shown more clearly. In the end, the Diol does not supply adequate retention or resolution for any useful separations with these RP compounds.

\subsubsection{Acidic, Basic and Neutral Compounds}

A small set of common acidic, basic and neutral compounds were employed for additional characterization of the four columns using both Agilent 1200 HPLCs. Trends shown in Table 8 correspond to the $\log \mathrm{P}$ values for the proposed compounds. Table 9 shows that Trends 1 and 2 are followed except Trend 3 where anisole gave higher retention than ethylbenzene. Anisole has a significantly lower logP value than ethylbenzene (1.99 vs. 2.85) due to the presence of the methoxy group and the reason for anisole’s higher retention is unclear. Amitriptyline and diphenhydramine were the only two that displayed ANP retention from $90 \% \mathrm{~B}-70 \% \mathrm{~B}$, followed by a switch to RP mode from $60 \%$ B - 10\% B. The retention map below (Figure 19) shows that significant retention can be achieved for all six compounds. Finally, the overlaid chromatograms (Figure 20) display the Diamond C18's successful resolution of each compound as 
organic mobile phase is reduced. Since amitriptyline and diphenhydramine experience band broadening at lower organic compositions gradient separations would need to be used to achieve full resolution.

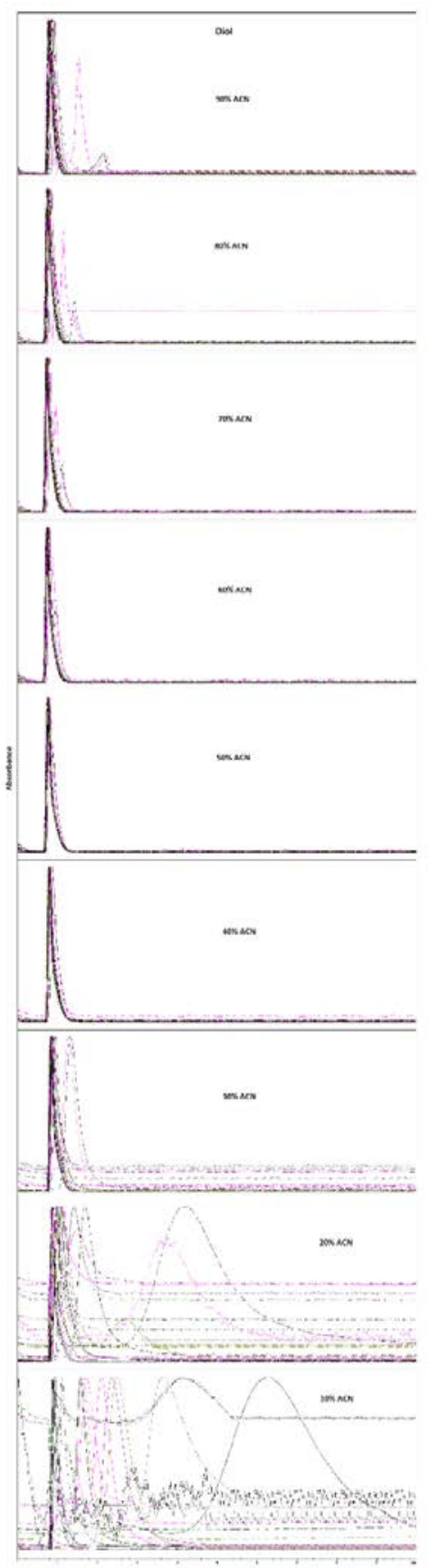

Figure 18. Diol with Acetonitrile Mobile Phase Overlaid/Stacked Chromatograms. 
Table 8. Retention trends of acidic/basic/neutral compounds based on structure and polarity.

\begin{tabular}{|c|c|c|}
\hline Trend \# & Reversed Phase & Aqueous Normal Phase \\
\hline $\mathbf{1}$ & Ketoprofen $>$ 2-Nitrobenzoic Acid & 2-Nitrobenzoic Acid $>$ Ketoprofen \\
\hline $\mathbf{2}$ & Amitriptyline $>$ Diphenhydramine & Diphenhydramine $>$ Amitriptyline \\
\hline $\mathbf{3}$ & Naphthalene $>$ Ethylbenzene $>$ Anisole & Anisole $>$ Ethylbenzene $>$ Naphthalene \\
\hline
\end{tabular}

Table 9. Diamond Bidentate C18 Capacity Factor “ $k$ ” (ACN/ABN). Average of in triplicate injections from $10 \%$ organic to $90 \%$ organic.

\begin{tabular}{|c|c|c|c|c|c|c|c|c|}
\hline & \multicolumn{8}{|c|}{ Diamond Bidentate C18 } \\
\hline & \multirow{2}{*}{ Acidic/Basic/Neutral } & \multicolumn{7}{|c|}{ Mobile Phase Composition (\% Acetonitrile) } \\
\hline & & 30 & 40 & 50 & 60 & 70 & 80 & 90 \\
\hline \multirow{7}{*}{ 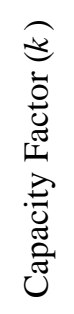 } & Ketoprofen & 17.34 & 4.84 & 2.01 & 0.98 & 0.6 & 0.39 & 0.26 \\
\hline & 2-Nitrobenzoic Acid & 1.33 & 0.78 & 0.49 & 0.31 & 0.26 & 0.17 & 0.13 \\
\hline & Amitriptyline & 17.52 & 5.67 & 3.03 & 2.16 & 2.05 & 2.45 & 3.67 \\
\hline & Diphenhydramine & 5.91 & 2.48 & 1.55 & 1.23 & 1.27 & 1.68 & 2.73 \\
\hline & Anisole & 13.68 & 6.46 & 3.44 & 1.92 & 1.23 & 0.82 & 0.53 \\
\hline & Naphthalene & & 23.5 & 10.12 & 4.81 & 2.75 & 1.65 & 0.97 \\
\hline & Ethylbenzene & 5.58 & 2.89 & 1.71 & 1.08 & 0.75 & 0.54 & 0.39 \\
\hline
\end{tabular}

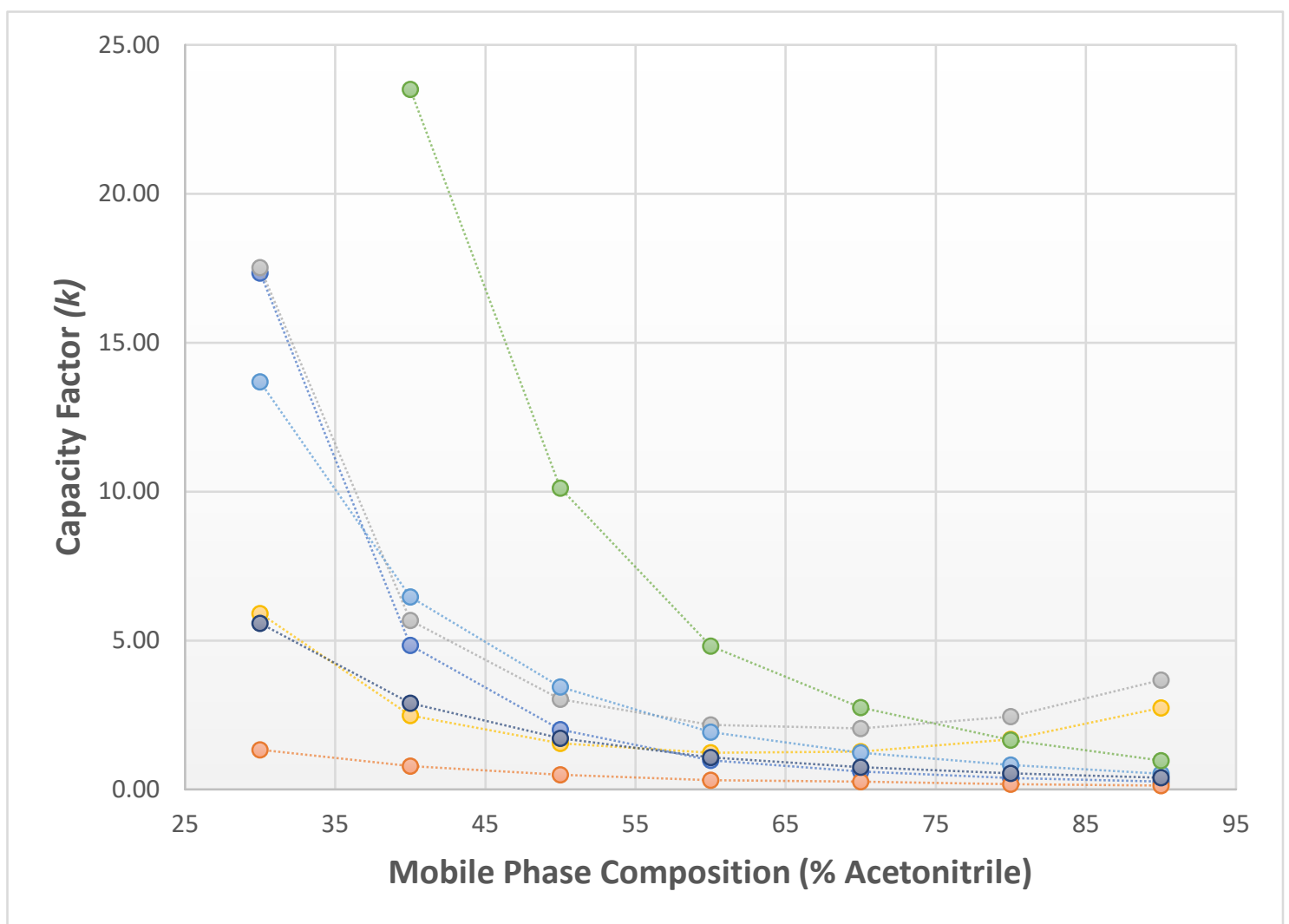

Figure 19. Diamond C18 Retention Map (ACN/ABN). Compares capacity factor " $k$ " ( $y$ axis) to percentage acetonitrile mobile phase (x-axis). 


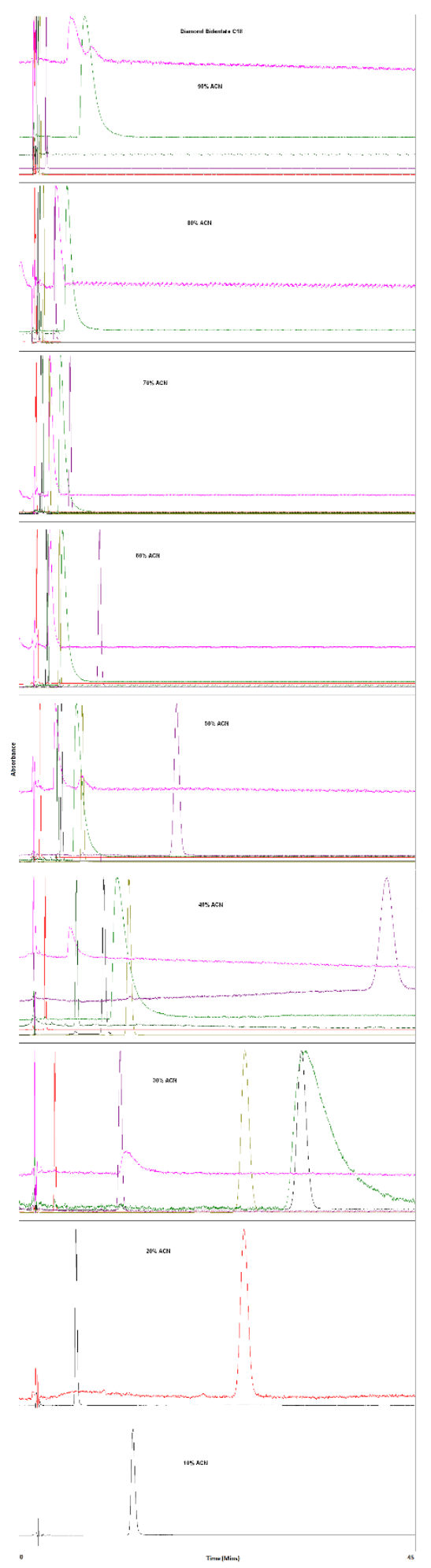

Figure 20. Diamond Bidentate C18 with Acetonitrile Mobile Phase Overlaid/Stacked Chromatograms. 
The Bidentate C18 column demonstrated the same retention patterns as the Diamond C18. The main difference observed was that all the compounds were retained slightly more on the Bidentate C18 than the hybrid column (Table 10). Selectivity and resolution were also comparable as shown in Figure 22. The Bidentate C18 also showed higher ANP properties for amitriptyline and diphenhydramine than the Diamond C18 (Figure 21).

Table 10. Bidentate C18 Capacity Factor " $k$ ” (ACN/ABN). Average of in triplicate injections from $10 \%$ to $90 \%$ organic.

\begin{tabular}{|c|c|c|c|c|c|c|c|c|}
\hline & \multicolumn{8}{|c|}{ Bidentate C18 } \\
\hline & \multirow{2}{*}{ Acidic/Basic/Neutral } & \multicolumn{7}{|c|}{ Mobile Phase Composition (\% Acetonitrile) } \\
\hline & & 10 & 20 & 30 & 40 & 50 & 60 & 70 \\
\hline \multirow{7}{*}{ 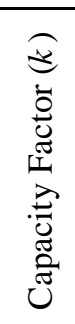 } & Ketoprofen & & & 21.65 & 5.79 & 2.43 & 1.14 & 0.66 \\
\hline & 2-Nitrobenzoic Acid & 7.15 & 3.15 & 1.68 & 0.97 & 0.6 & 0.36 & 0.28 \\
\hline & Amitriptyline & & & 20.41 & 6.67 & 3.83 & 3 & 2.49 \\
\hline & Diphenhydramine & & & 7.04 & 3.04 & 2.13 & 1.66 & 1.57 \\
\hline & Anisole & & & 17.74 & 8.13 & 4.28 & 2.28 & 1.39 \\
\hline & Naphthalene & & & & & 12.43 & 5.58 & 3.11 \\
\hline & Ethylbenzene & & 18.32 & 7.64 & 3.88 & 2.26 & 1.34 & 0.9 \\
\hline
\end{tabular}

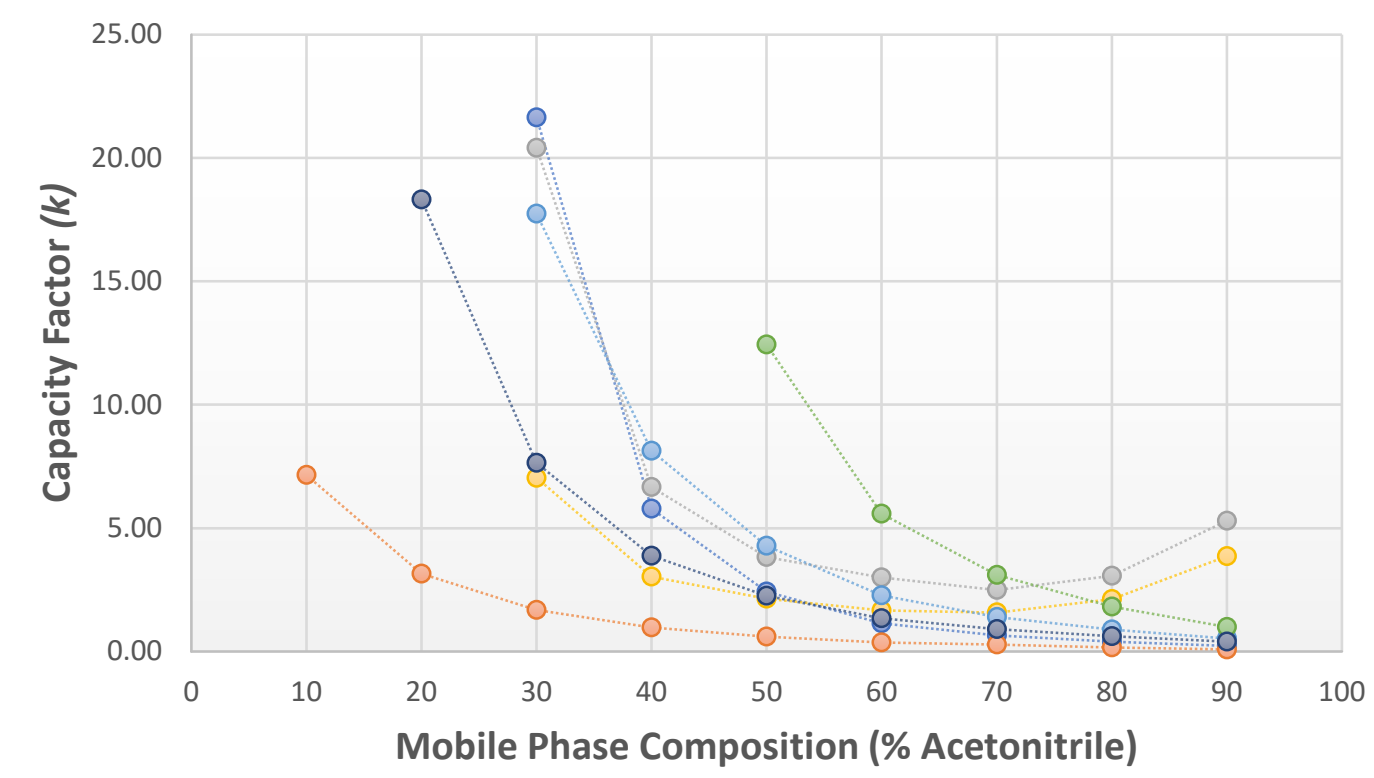

Figure 21. Bidentate C18 Retention Map (ACN/ABN). Compares capacity factor " $k$ " (yaxis) to percentage acetonitrile mobile phase (x-axis). 


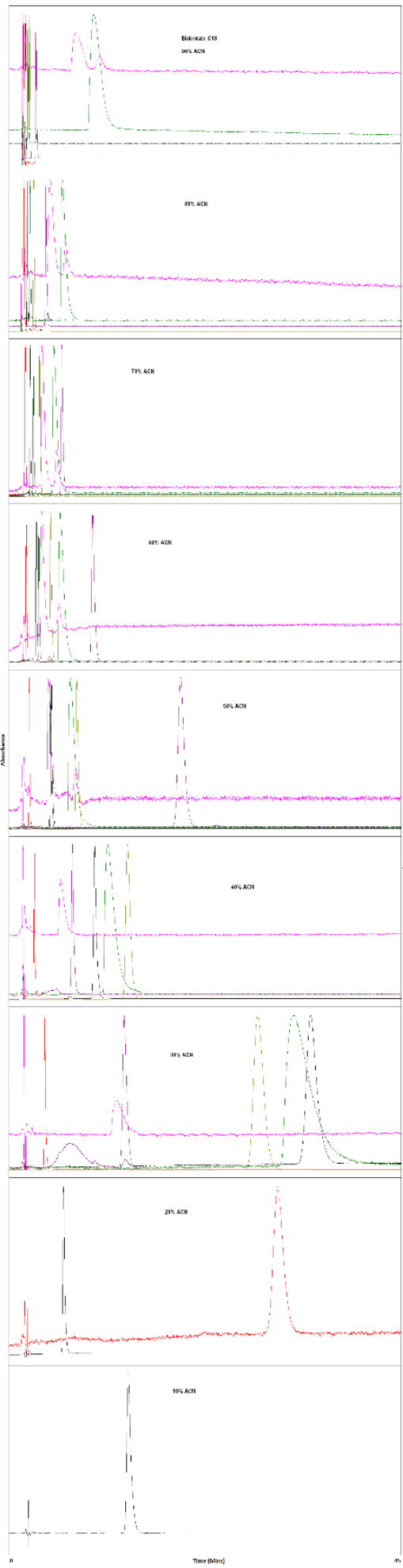

Figure 22. Bidentate C18 with Acetonitrile Mobile Phase Overlaid/Stacked Chromatograms. 
The Phenyl Hydride demonstrated the same reversed phased retention as the Diamond C18 and Bidentate C18. Similar to the other two, the Phenyl followed Trends 1 and 2 but still showed greater retention of anisole over ethylbenzene in Trend 3. The main differences were that the retention power of the Phenyl Hydride was much lower for these compounds (Table 11) but the peak shape for the compounds gave less peak broadening that the other two reversed phase columns (Figure 24). In addition, the ANP properties demonstrated by retention of amitriptyline and diphenhydramine were much weaker than the Diamond C18 and Bidentate C18 stationary phases (Figure 23).

Table 11. Phenyl Hydride Capacity Factor " $k$ ” (ACN/ABN). Average of in triplicate injections from $10 \%$ to $90 \%$ organic.

\begin{tabular}{|c|c|c|c|c|c|c|c|c|}
\hline & \multicolumn{8}{|c|}{ Phenyl Hydride } \\
\hline & \multirow{2}{*}{ Acidic/Basic/Neutral } & \multicolumn{7}{|c|}{ Mobile Phase Composition (\% Acetonitrile) } \\
\hline & & 10 & 20 & 30 & 40 & 50 & 60 & 70 \\
\hline \multirow{7}{*}{ 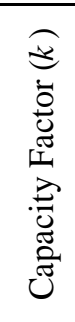 } & Ketoprofen & & 33.35 & 7.67 & 2.71 & 1.14 & 0.48 & 0.37 \\
\hline & 2-Nitrobenzoic Acid & 2.83 & 1.89 & 1.17 & 0.73 & 0.44 & 0.22 & 0.23 \\
\hline & Amitriptyline & & 18.28 & 3.72 & 1.29 & 0.59 & 0.32 & 0.4 \\
\hline & Diphenhydramine & 26.26 & 5.63 & 1.57 & 0.62 & 0.33 & 0.18 & 0.29 \\
\hline & Anisole & 13.65 & 8.13 & 4.35 & 2.33 & 1.25 & 0.61 & 0.49 \\
\hline & Naphthalene & & 35.68 & 13.17 & 5.43 & 2.43 & 1.08 & 0.75 \\
\hline & Ethylbenzene & 11.07 & 5.05 & 2.61 & 1.48 & 0.88 & 0.48 & 0.43 \\
\hline
\end{tabular}

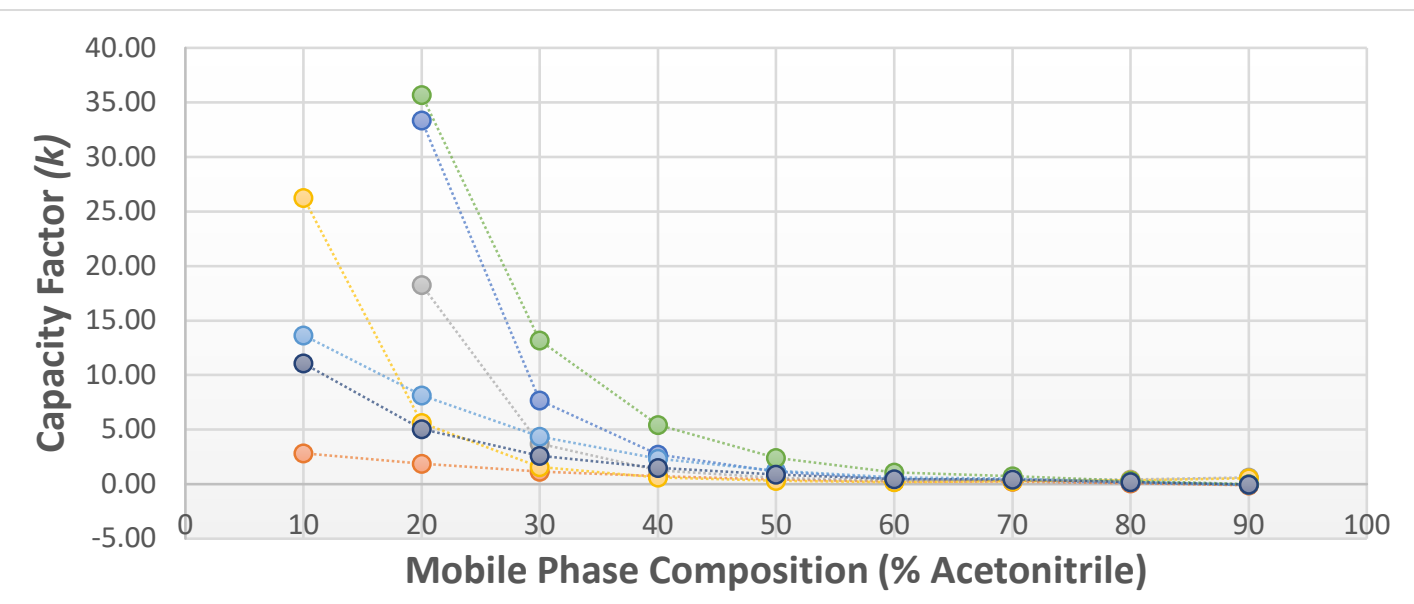

Figure 23. Phenyl Hydride Retention Map (ACN/ABN). Compares capacity factor “ $k$ ” (y-axis) to percentage acetonitrile mobile phase (x-axis). 


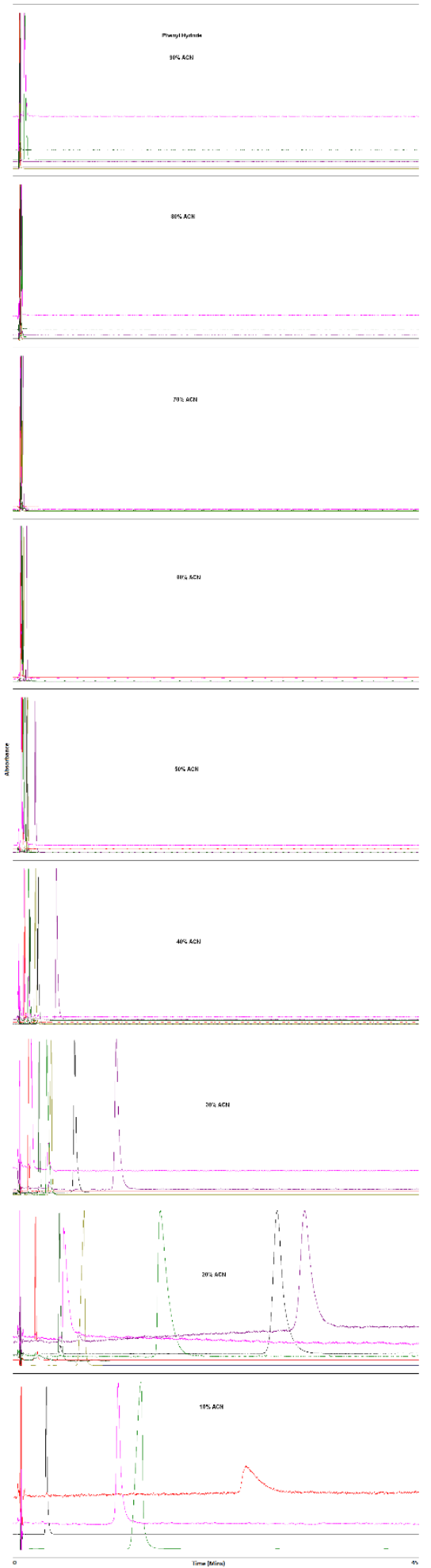

Figure 24. Phenyl Hydride with Acetonitrile Mobile Phase Overlaid/Stacked Chromatograms. 
Finally, the Diol column was the only column to follow all three expected trend orders. The retention map (Table 12) shows typical RP retention from high to lower organic for the acidic and neutral species but demonstrates both ANP and RP retention for amitriptyline and diphenhydramine. The ANP properties are much more apparent for the Diol stationary phase than the others but the retention power and selectivity are the lowest of the four (Figure 25 and 26). The resveratrol compounds do not appear to have enough affinity for the Diol column to be separated as a mixture.

Table 12. Diol Capacity Factor " $k$ " (ACN/ABN). Average of in triplicate injections from $10 \%$ to $90 \%$ organic.

\begin{tabular}{|c|c|c|c|c|c|c|c|c|}
\hline & \multicolumn{8}{|c|}{ Diol } \\
\hline & \multirow{2}{*}{ Acidic/Basic/Neutral } & \multicolumn{7}{|c|}{ Mobile Phase Composition (\% Acetonitrile) } \\
\hline & & 10 & 20 & 30 & 40 & 50 & 60 & 70 \\
\hline \multirow{7}{*}{ 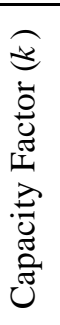 } & Ketoprofen & 1.92 & 0.29 & 0.08 & -0.01 & -0.07 & -0.14 & -0.21 \\
\hline & 2-Nitrobenzoic Acid & 0.14 & 0.09 & 0.04 & 0 & -0.04 & -0.1 & -0.18 \\
\hline & Amitriptyline & 1.75 & 0.39 & 0.15 & 0.06 & 0.01 & -0.02 & -0.03 \\
\hline & Diphenhydramine & 0.74 & 0.25 & 0.11 & 0.06 & 0.01 & -0.01 & -0.01 \\
\hline & Anisole & 0.47 & 0.23 & 0.1 & 0.02 & -0.05 & -0.11 & -0.2 \\
\hline & Naphthalene & 2.14 & 0.69 & 0.22 & 0.04 & -0.05 & -0.14 & -0.21 \\
\hline & Ethylbenzene & 0.56 & 0.24 & 0.11 & 0.03 & -0.03 & -0.1 & -0.18 \\
\hline
\end{tabular}

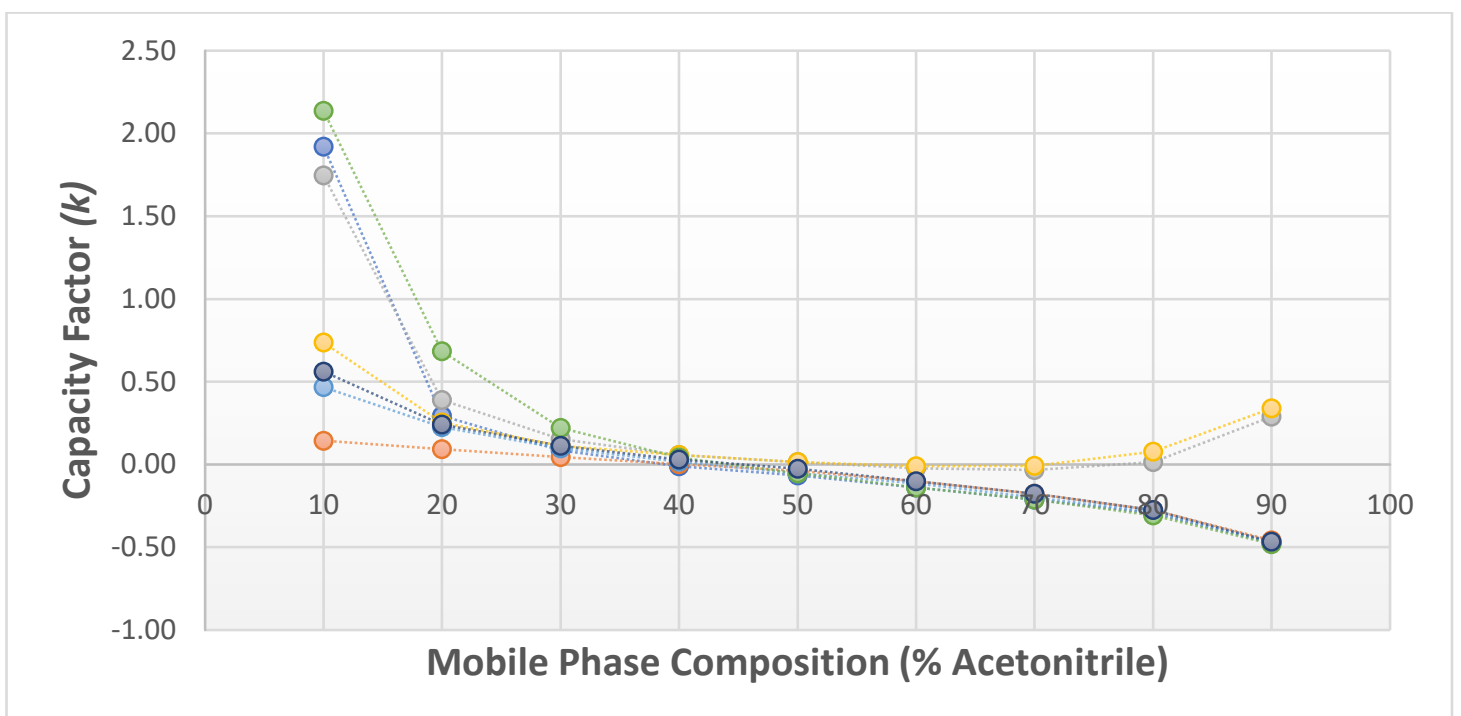

Figure 25. Diol Retention Map (ACN/ABN). Compares capacity factor “ $k$ ” (y-axis) to percentage acetonitrile mobile phase ( $\mathrm{x}$-axis). 


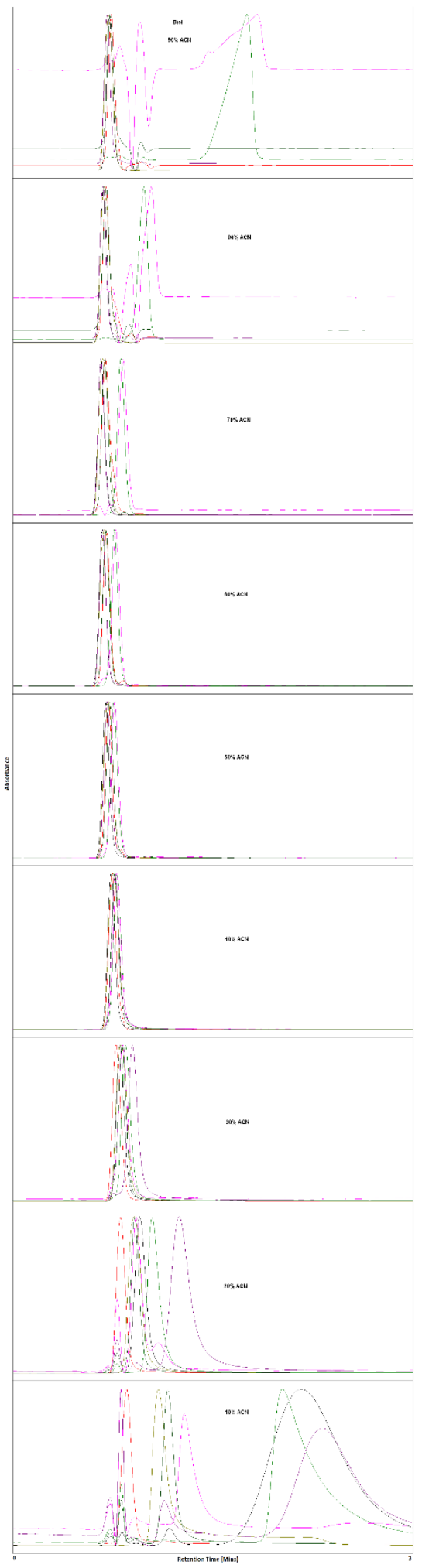

Figure 26. Diol with Acetonitrile Mobile Phase Overlaid/Stacked Chromatograms 
In terms of retention power when using acetonitrile as mobile phase, the Bidentate C18 is the highest, followed by the Diamond C18, then the Phenyl Hydride and finally the Diol. The Phenyl Hydride demonstrates the most selectivity and resolution compared to the other three (Bidentate and Diamond C18 are comparable). The reason for this may be due to the additional pi-pi interactions with the aromatics and phenolic aspects of the resveratrol analogues.

\subsection{Data with Methanol as Mobile Phase}

\subsubsection{Resveratrol Analogues}

When comparing the mobile phases, the first expected difference is in retention. Since methanol is a weaker organic than acetonitrile which supports that higher retention is assumed when using methanol. This concept is fully supported for all four columns when comparing the methanol retention maps to the acetonitrile ones for the resveratrol analogues (Tables 13-16). When specifically observing the Diamond C18, three differences between trend order are noted. The first difference was that Trends 1 and 2 (amide vs. imine/amine) showed the amide resveratrol retained longer than the imine and amine versions while in acetonitrile this was not the case. The second change was seen between the meta/para position resveratrol isomers (MH17 and 18) where in acetonitrile MH18 displays greater retention than MH17 while in methanol the order is flipped. Surprisingly, although retention is greater in methanol, selectivity for the meta/para isomers is lower when compared to acetonitrile mobile phase. The third variation was observed between MH14 and MH15 (1, 2, 3-triol and 1, 3-diol) where acetonitrile promoted retention such that MH14 > MH15 while methanol supported the opposite 
order. Reasons for this change are unclear at this time. When referring to retention power, 20 of the 22 compounds gave a capacity factor $k>2$ supporting increased retention with methanol. In terms of selectivity, Figures 27 and 28 confirm that methanol gives better selectivity and resolution than acetonitrile for these resveratrol analogues.

Table 13. Diamond Bidentate C18 Capacity Factor “ $k$ ” (MeOH/Resveratrol). Average of in triplicate injections from $10 \%$ to $90 \%$ organic.

\begin{tabular}{|c|c|c|c|c|c|c|c|c|}
\hline & \multicolumn{8}{|c|}{ Diamond Bidentate C18 } \\
\hline & \multirow{2}{*}{ Resveratrol Analogues } & \multicolumn{7}{|c|}{ Mobile Phase Composition (\% Methanol) } \\
\hline & & 10 & 20 & 30 & 40 & 50 & 60 & 70 \\
\hline \multirow{22}{*}{ 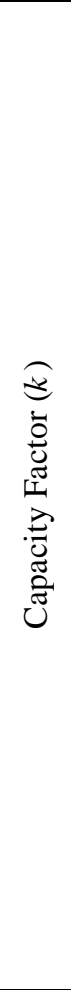 } & MH 1 & & & 16.62 & 4.41 & 1.25 & 0.42 & 0.16 \\
\hline & MH 2 & 6.54 & 2.6 & 1.31 & 0.66 & 0.32 & 0.1 & 0.07 \\
\hline & MH 3 & & & 1.89 & 0.67 & 0.25 & 0.1 & 0.03 \\
\hline & MH 4 & & & 7.02 & 2.13 & 0.67 & 0.12 & 0.01 \\
\hline & MH 5 & & & & & 6.38 & 2 & 0.76 \\
\hline & MH 7 & & & & & 10.96 & 2.8 & 1.05 \\
\hline & MH 8 & & & & & 8.07 & 2.15 & 0.8 \\
\hline & MH 9 & & & & & & 12.56 & 4.41 \\
\hline & MH 10 & & & & & & & 20.77 \\
\hline & MH 11 & & & 16.9 & 4.88 & 1.51 & 0.5 & 0.2 \\
\hline & MH 12 & 2.4 & 1.34 & 0.93 & 0.66 & 0.33 & 0.39 & 0.08 \\
\hline & MH 13 & & & & 6.85 & 1.79 & 0.41 & 0.19 \\
\hline & MH 14 & & & & & 5.79 & 2.16 & 0.92 \\
\hline & MH 15 & & & & & 9.46 & 2.68 & 1.02 \\
\hline & MH 16 & & & & & 8.85 & 2.27 & 0.86 \\
\hline & MH 17 & & & & & & 11.46 & 3.9 \\
\hline & MH 18 & & & & & & 11.19 & 3.76 \\
\hline & MH 19 & & & & & & & 19.46 \\
\hline & MH 20 & & & 11.04 & 1.72 & 0.37 & 0.07 & 0 \\
\hline & MH 21 & 1.33 & 0.56 & 0.29 & 0.15 & 0.07 & 0.03 & 0.01 \\
\hline & MH 22 & 12.42 & 2.45 & 0.81 & 0.27 & 0.1 & 0.03 & 0 \\
\hline & MH 23 & & & & 9 & 2.7 & 0.81 & 0.33 \\
\hline
\end{tabular}


Table 14. Bidentate C18 Capacity Factor " $k$ " (MeOH/Resveratrol). Average of in triplicate injections from $10 \%$ to $90 \%$ organic.

\begin{tabular}{|c|c|c|c|c|c|c|c|c|}
\hline & \multicolumn{8}{|c|}{ Bidentate C18 } \\
\hline & \multirow{2}{*}{ Resveratrol Analogues } & \multicolumn{7}{|c|}{ Mobile Phase Composition (\% Methanol) } \\
\hline & & 10 & 20 & 30 & 40 & 50 & 60 & 70 \\
\hline \multirow{22}{*}{ 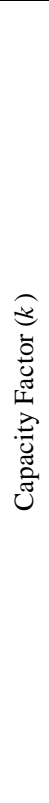 } & MH 1 & & & 20.7 & 5.49 & 1.73 & 0.58 & 0.25 \\
\hline & MH 2 & 7.96 & 3.33 & 1.58 & 0.81 & 0.44 & 0.26 & 0.18 \\
\hline & MH 3 & & 8.32 & 2.31 & 0.84 & 0.36 & 0.17 & 0.09 \\
\hline & MH 4 & & & & & 0.88 & 0.42 & 0.23 \\
\hline & MH 5 & & & & & 7.64 & 2.99 & 1.24 \\
\hline & MH 7 & & & & & 12.73 & 3.52 & 1.29 \\
\hline & MH 8 & & & & & 10 & 2.79 & 1.06 \\
\hline & MH 9 & & & & & & 16.89 & 5.49 \\
\hline & MH 10 & & & & & & & 26.38 \\
\hline & MH 11 & & & 20.89 & 5.96 & 1.95 & 0.66 & 0.29 \\
\hline & MH 12 & 7.89 & 3.33 & 1.58 & 0.81 & 0.44 & 0.25 & 0.17 \\
\hline & MH 13 & & & & 3.15 & 1.25 & 0.54 & 0.28 \\
\hline & MH 14 & & & & & 7.17 & 2.55 & 1.14 \\
\hline & MH 15 & & & & & 11.19 & 3.29 & 1.24 \\
\hline & MH 16 & & & & & 9.93 & 2.87 & 1.1 \\
\hline & MH 17 & & & & & & 14.48 & 4.82 \\
\hline & MH 18 & & & & & & 14.11 & 4.62 \\
\hline & MH 19 & & & & & & & 24.18 \\
\hline & MH 20 & & & 13.53 & 2.2 & 0.52 & 0.14 & 0.06 \\
\hline & MH 21 & 1.67 & 0.74 & 0.38 & 0.21 & 0.13 & 0.08 & 0.06 \\
\hline & MH 22 & 14.54 & 3.33 & 0.99 & 0.36 & 0.17 & 0.08 & 0.06 \\
\hline & MH 23 & & & & 10.81 & 3.38 & 1.07 & 0.46 \\
\hline
\end{tabular}

Table 15. Phenyl Hydride Capacity Factor " $k$ ” (MeOH/Resveratrol). Average of in triplicate injections from $10 \%$ to $90 \%$ organic.

\begin{tabular}{|c|c|c|c|c|c|c|c|c|}
\hline & \multicolumn{8}{|c|}{ Phenyl Hydride } \\
\hline & \multirow{2}{*}{ Resveratrol Analogues } & \multicolumn{7}{|c|}{ Mobile Phase Composition (\% Methanol) } \\
\hline & & 10 & 20 & 30 & 40 & 50 & 60 & 70 \\
\hline \multirow{22}{*}{ 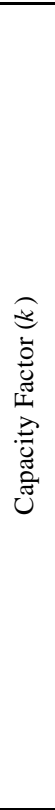 } & MH 1 & & 24.55 & 7.26 & 2.12 & 0.3 & -0.35 & -0.43 \\
\hline & MH 2 & 1.73753 & 0.83 & 0.25 & -0.08 & -0.3 & -0.47 & -0.47 \\
\hline & MH 3 & 6.03448 & 2.24 & 0.63 & 0.01 & -0.31 & -0.49 & -0.49 \\
\hline & MH 4 & 4.05 & 2.2 & 1.01 & 0.34 & -0.08 & -0.4 & -0.44 \\
\hline & MH 5 & & & 29.02 & 5.64 & 1.66 & 0.21 & -0.18 \\
\hline & MH 7 & & & 25.05 & 7.7 & 1.93 & 0.06 & -0.3 \\
\hline & MH 8 & & & 23.58 & 7.11 & 1.72 & 0.01 & -0.36 \\
\hline & MH 9 & & & & 24.27 & 6.16 & 0.94 & -0.18 \\
\hline & MH 10 & & & & & 17.39 & 3.1 & 0.52 \\
\hline & MH 11 & & 15.51 & 4.8 & 1.48 & 0.18 & -0.35 & -0.49 \\
\hline & MH 12 & 1.75 & 0.84 & 0.23 & -0.09 & -0.3 & -0.47 & -0.53 \\
\hline & MH 13 & 36.403 & 17.83 & 2.87 & 0.92 & 0.09 & -0.33 & -0.46 \\
\hline & MH 14 & & & 17.49 & 3.92 & 1.19 & 0.03 & -0.31 \\
\hline & MH 15 & & & 16.1 & 5.41 & 1.42 & -0.02 & -0.37 \\
\hline & MH 16 & & & 16.45 & 5.41 & 1.36 & -0.04 & -0.42 \\
\hline & MH 17 & & & & 18.8 & 5 & 0.76 & -0.18 \\
\hline & MH 18 & & & & 17.43 & 4.7 & 0.71 & -0.19 \\
\hline & MH 19 & & & & & 15.22 & 2.76 & 0.34 \\
\hline & MH 20 & & & 22.64 & 3.95 & 0.39 & -0.38 & -0.56 \\
\hline & MH 21 & 0.22086 & -0.04 & -0.23 & -0.35 & -0.44 & -0.53 & -0.6 \\
\hline & MH 22 & 3.699 & 1.25 & 0.22 & -0.18 & -0.38 & -0.52 & -0.59 \\
\hline & MH 23 & & & 12.93 & 3.72 & 0.77 & -0.2 & -0.48 \\
\hline
\end{tabular}


Table 16. Diol Capacity Factor " $k$ ” (MeOH/Resveratrol). Average of in triplicate injections from $10 \%$ to $90 \%$ organic.

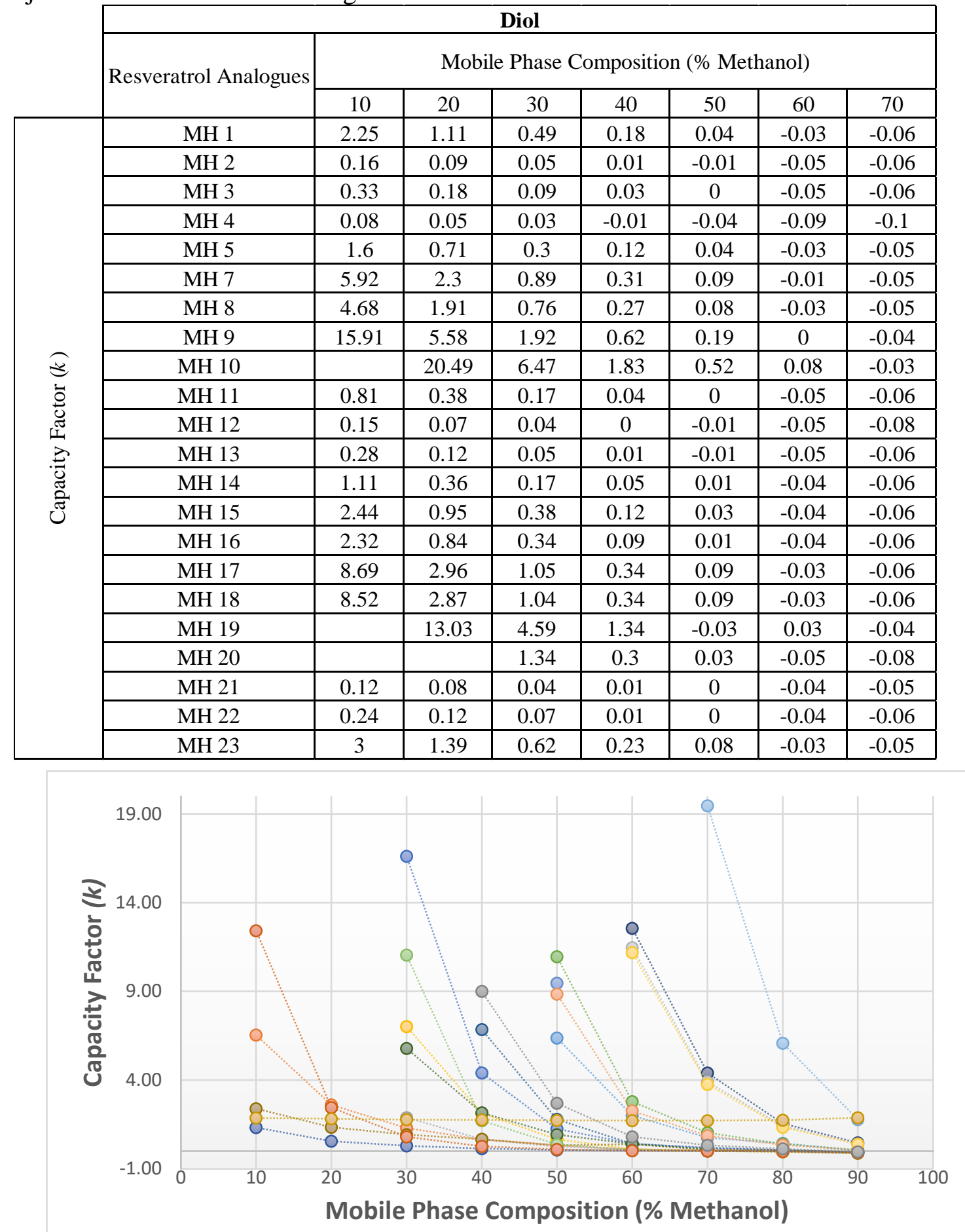

Figure 27. Diamond C18 Retention Map (MeOH/Resveratrol). Compares capacity factor " $k$ " (y-axis) to percentage mobile phase (x-axis). 


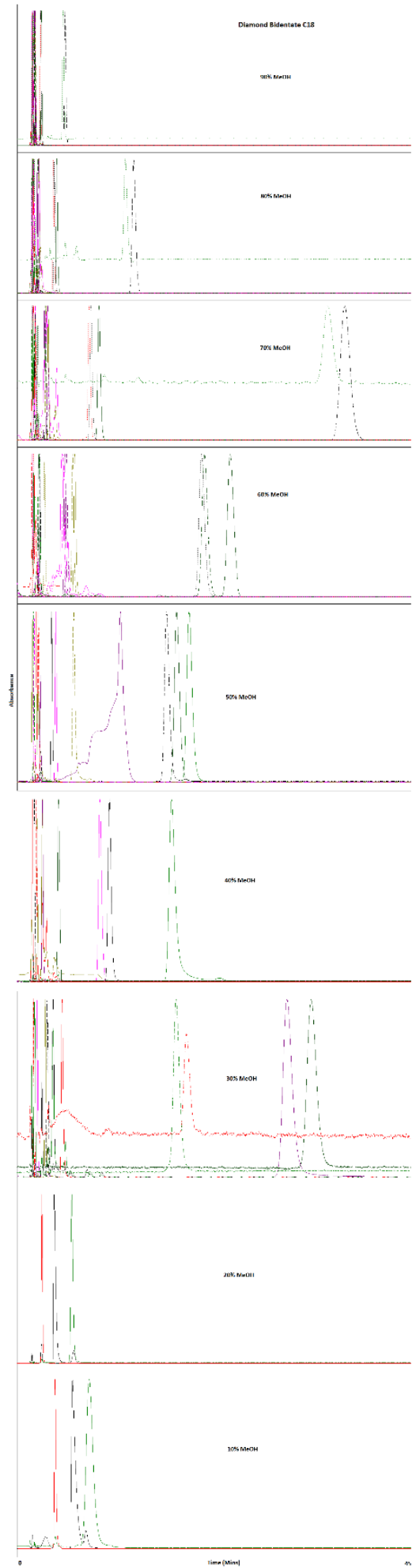

Figure 28. Diamond Bidentate C18 with Methanol Mobile Phase Overlaid/Stacked Chromatograms. 
The Bidentate C18 followed similar RP trends as the Diamond C18 and also showed significant retention of 20 out of the 22 compounds $(k>2)$. In terms of retention power, the Bidentate C18 showed slightly higher retention for all the compounds than the Diamond C18. Similar to the Diamond C18, the Bidentate showed more retention of MH3 than MH2/MH12 (Trends 1 and 2) at higher water mobile phase compositions. The second change was observed for Trend 7 where the expected trend was followed in methanol but completely different in acetonitrile. No concrete explanation for this phenomenon has been determined. The last difference found in methanol was the retention order of MH1, MH20 and MH23 (Synthetic vs. Natural Derivatives). In acetonitrile MH20 retained more than MH23 and MH1 while in methanol the order of retention was MH23 > MH1 > MH20. Despite these differences in retention order, the selectivity of the Bidentate C18 was comparable to the Diamond C18 (Figures 29 and 30) but the Diamond C18 demonstrated higher selectivity of the unsaturated and saturated forms of resveratrol.

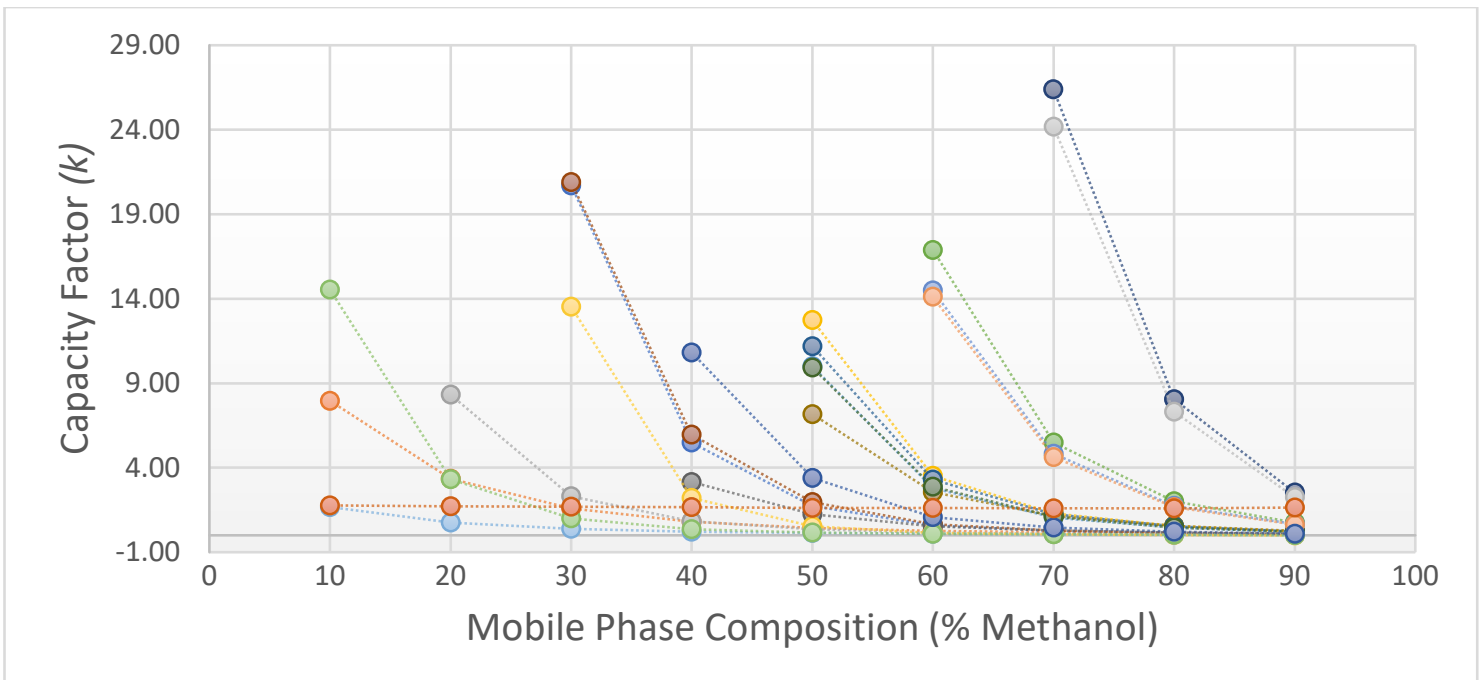

Figure 29. Bidentate C18 Retention Map (MeOH/Resveratrol). Compares capacity factor " $k$ " (y-axis) to percentage mobile phase (x-axis). 


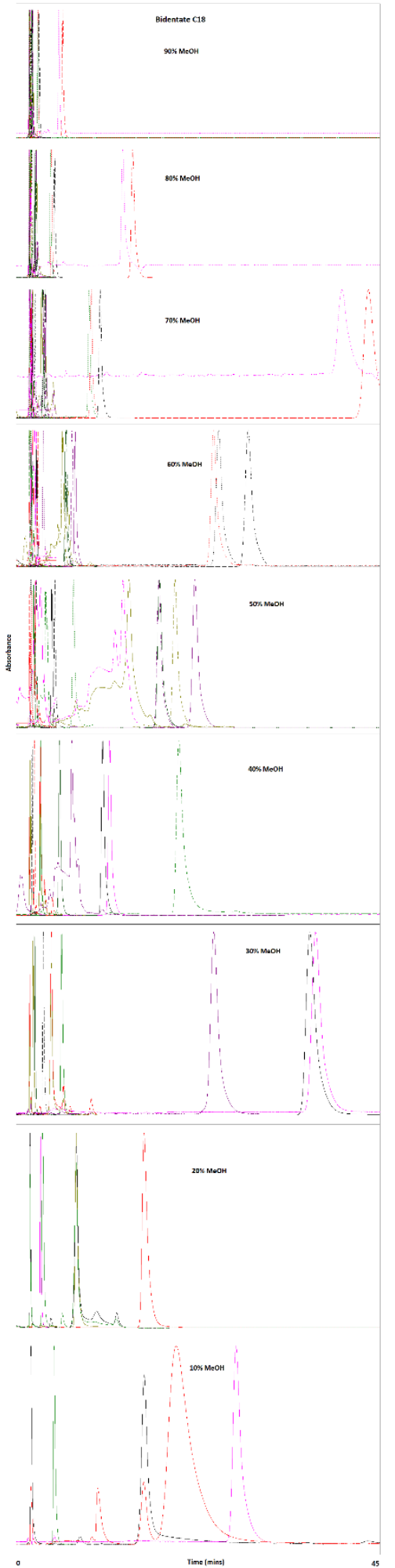

Figure 30. Bidentate C18 with Methanol Mobile Phase Overlaid/Stacked Chromatograms. 
The Phenyl Hydride column gave comparable retention orders as the previous RP columns and demonstrated the third highest retention of the four stationary phases. Comparing retention between acetonitrile and methanol, both solvents gave significant retention ( $k>2$ ) of 19 of the 22 compounds (Figure 31). In reference to the expected retention trends, there were four variations with methanol as solvent. First, the Phenyl column was the only one to follow Trend 4 when using methanol. The second and most important was the selectivity for the meta/para hydroxides (MH17 and MH18). The Phenyl Hydride demonstrated the highest selectivity for these isomers when using methanol. The next difference observed was a higher selectivity for MH14 (triol) in acetonitrile where MH14 > MH17 while in methanol MH17 > MH14 in terms of retention. In both solvents retention of MH14 > MH15 (triol > diol). Lastly, in acetonitrile the retention of MH1 > MH20 > MH23 while in methanol the order was MH23 > MH1 > MH20. In terms of selectivity and resolution the Phenyl Hydride shows the most potential (Figure 32).

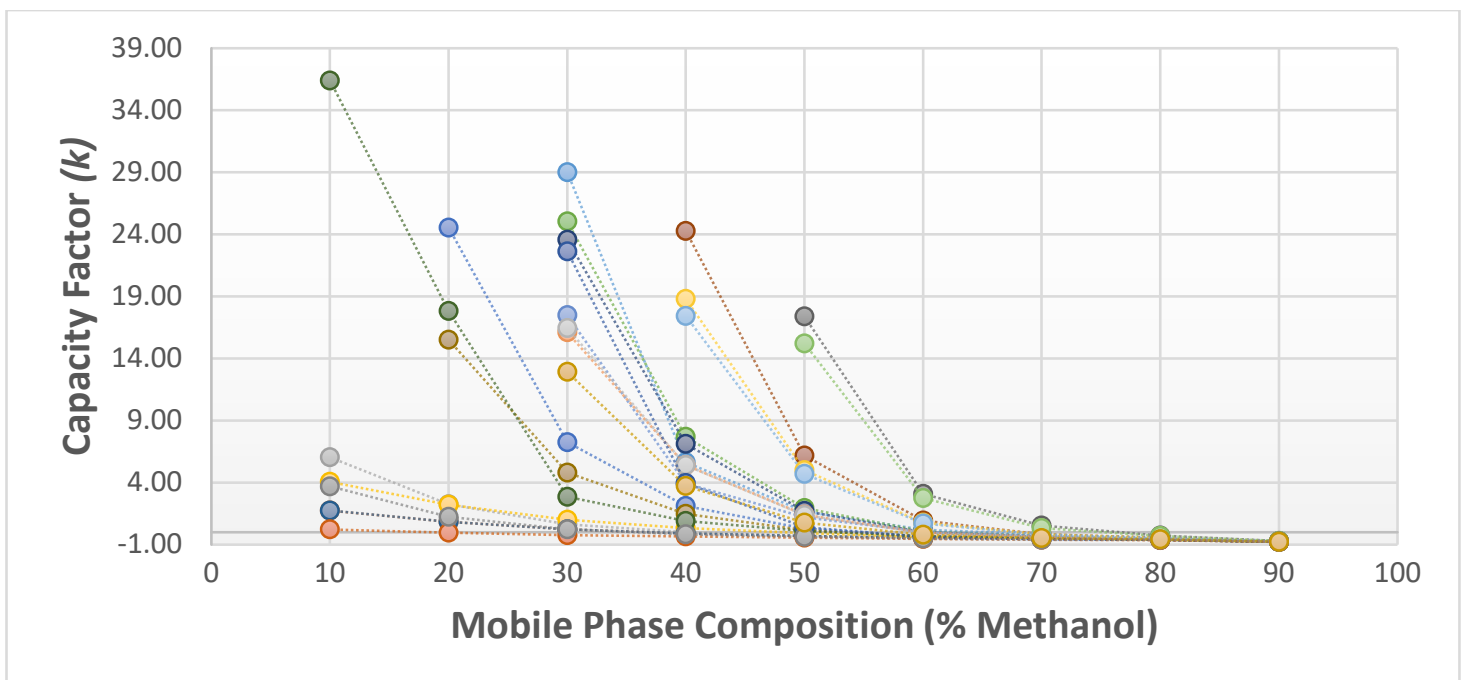

Figure 31. Phenyl Hydride Retention Map (MeOH/Resveratrol). Compares capacity factor " $k$ " (y-axis) to percentage mobile phase (x-axis). 


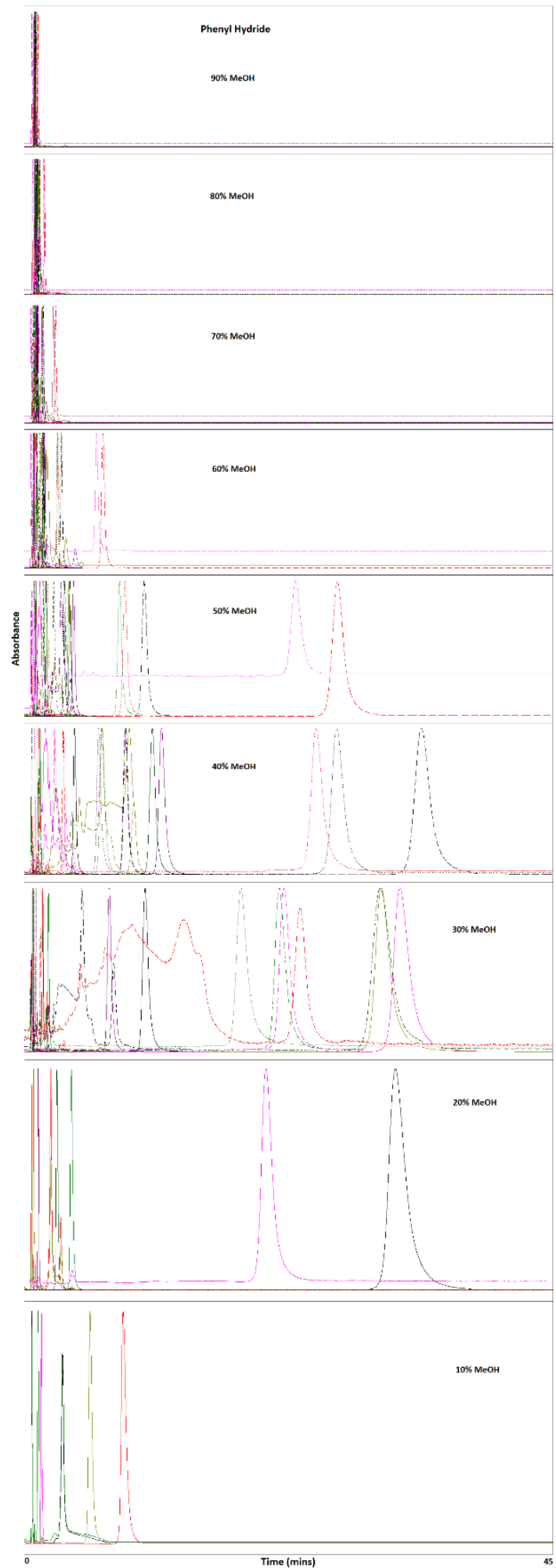

Figure 32. Phenyl Hydride with Methanol Mobile Phase Overlaid/Stacked Chromatograms. 
The Diol stationary phase still gave little to no retention for the majority of the resveratrol analogues despite the use of methanol mobile phase (Figure 33). In acetonitrile, eight of the 22 gave a $k>2$ compared to 11 of 22 in methanol. Although the selectivity was significant for half of the compounds, the selectivity was not high enough to afford acceptable resolution (Figure 34). Between the expected and actual retention trends only two differed between mobile phases. First, in acetonitrile the Diol followed the expected order for Trend 6, while in methanol retention of MH1 > MH5. Secondly, in acetonitrile the retention order for Trend 8 was actually MH11 > MH13 > MH14 whereas in methanol the original order was maintained. In terms of retention, selectivity and resolution the Diol stationary phase achieved little success. Interestingly, the ANP retention of MH2 was only observed in acetonitrile but not in methanol. No solid explanation can be given for these findings at this time.

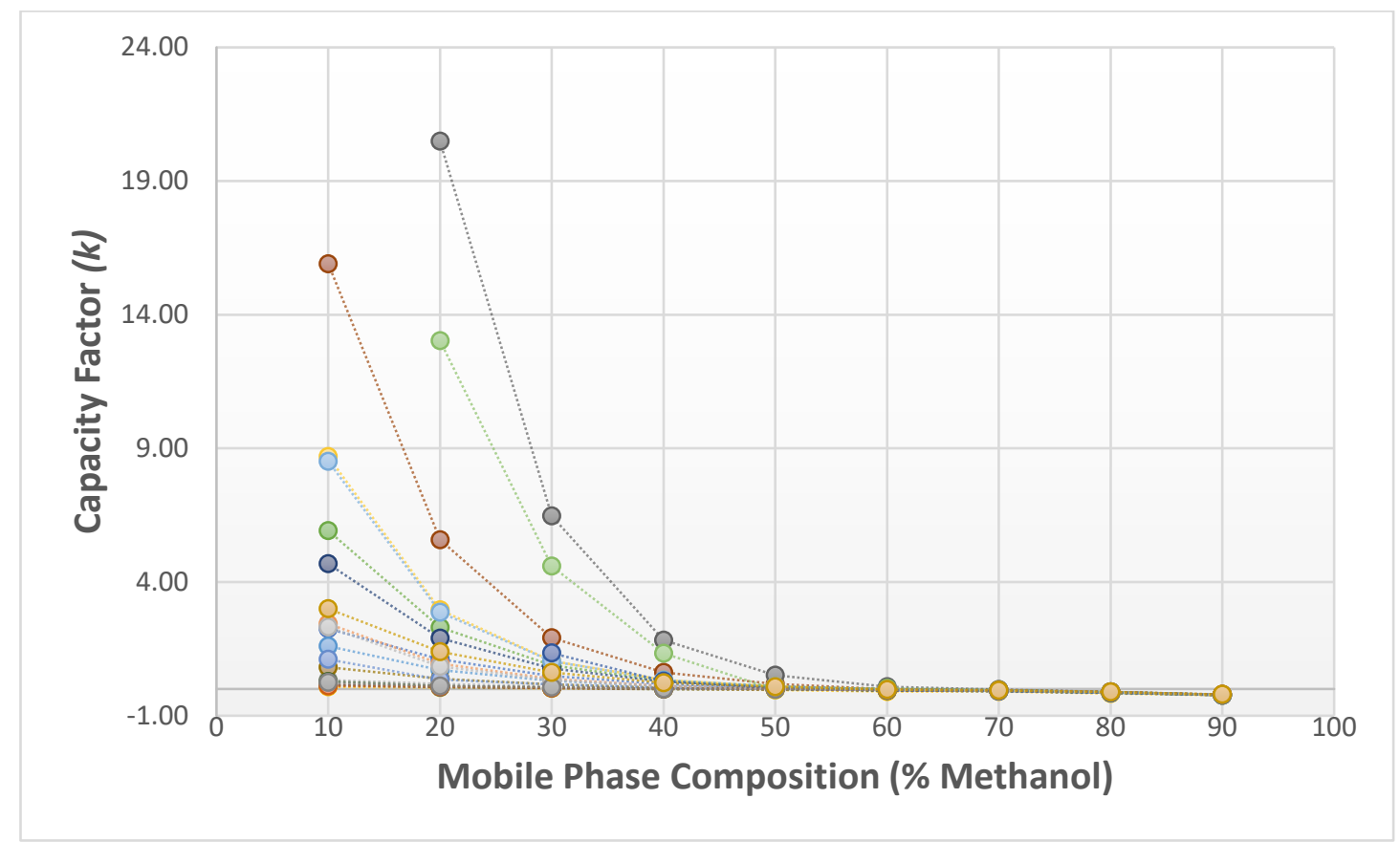

Figure 33. Diol Retention Map (MeOH/Resveratrol). Compares capacity factor " $k$ ” (yaxis) to percentage mobile phase (x-axis). 


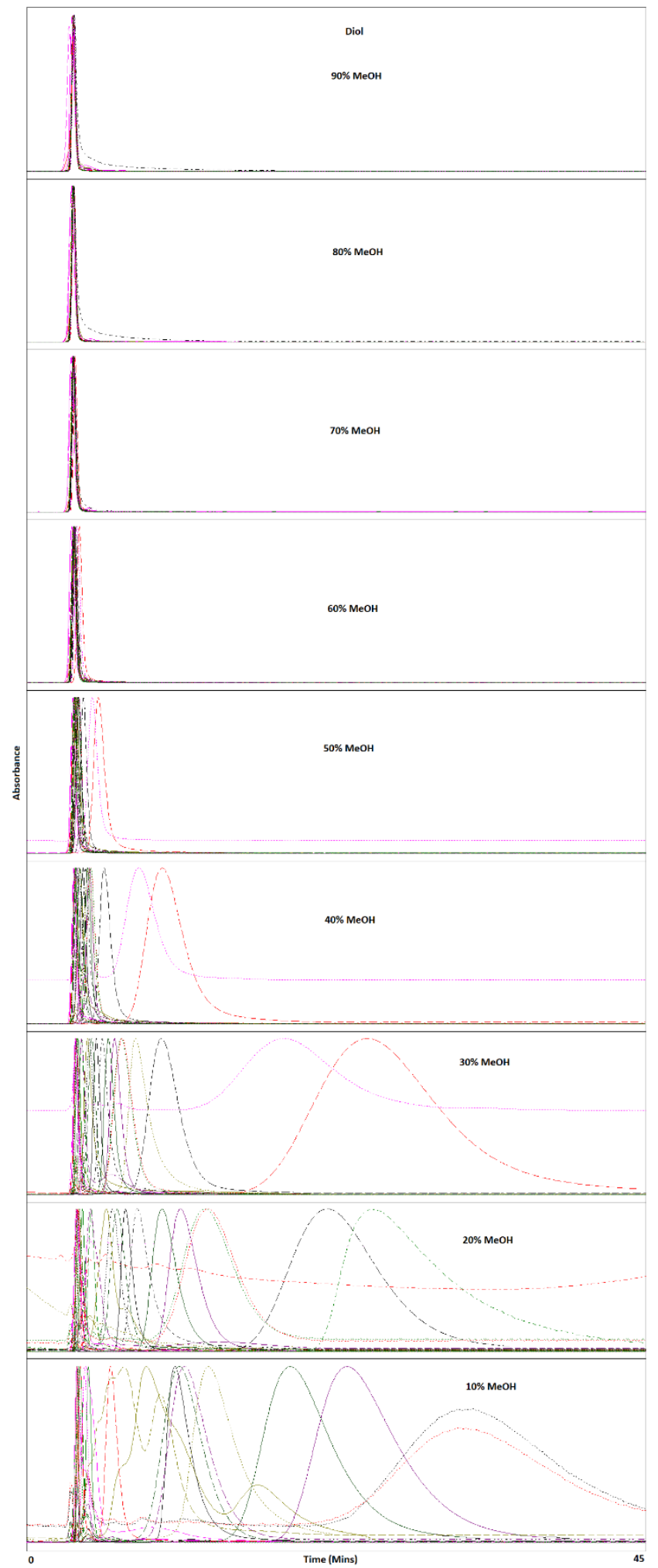

Figure 34. Diol with Methanol Mobile Phase Overlaid/Stacked Chromatograms. 
The retention strength of the columns only differed in methanol where the Bidentate C18 > Diamond C18. The selectivity for the resveratrol compounds was the same as in acetonitrile where Phenyl > Diamond C18 > Bidentate C18 > Diol. The main differences in methanol were higher retention of all compounds and the ANP properties observed for MH2 were only seen when acetonitrile was used as mobile phase. While there were significant differences in the expected retention trends

\subsubsection{Acidic, Basic and Neutral Compounds}

Similar results were found for the acidic, basic and neutral compounds when comparing the change in mobile phase solvent. Tables 17-20 support that methanol is a weaker organic solvent in that higher retention is observed for all seven compounds in RP mode with all four columns. The retention strength of the columns was the same as with acetonitrile (BC18 > DC18 > Phenyl > Diol) and the selectivity was similar as well. While the Diamond C18 and Bidentate C18 gave significant resolution, at lower organic content the peaks for amitriptyline and diphenhydramine broadened considerably (Figures 35 and 36).

Table 17. Diamond Bidentate C18 Capacity Factor “ $k$ ” (MeOH/ABN). Average of in triplicate injections from $10 \%$ to $90 \%$ organic.

\begin{tabular}{|c|c|c|c|c|c|c|c|c|c|c|}
\hline & \multicolumn{10}{|c|}{ Diamond Bidentate C18 } \\
\hline & \multirow{2}{*}{ Acidic/Basic/Neutral } & \multicolumn{9}{|c|}{ Mobile Phase Composition (\% Methanol) } \\
\hline & & 10 & 20 & 30 & 40 & 50 & 60 & 70 & 80 & 90 \\
\hline \multirow{7}{*}{ 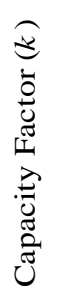 } & Ketoprofen & & & & & 14.71 & 3.89 & 1.37 & 0.45 & 0.14 \\
\hline & 2-Nitrobenzoic Acid & 7.72 & 4.28 & 2.45 & 1.35 & 0.75 & 0.36 & 0.19 & 0.08 & -0.03 \\
\hline & Amitriptyline & & & & & 12.09 & 3.21 & 1.25 & 0.56 & 0.33 \\
\hline & Diphenhydramine & & & & & 3.32 & 1.1 & 0.51 & 0.28 & 0.15 \\
\hline & Anisole & & & & & 9.32 & 4.12 & 2.09 & 0.91 & 0.46 \\
\hline & Naphthalene & & & & & & 15.51 & 6.28 & 2.16 & 1.02 \\
\hline & Ethylbenzene & & & & 9.26 & 4.36 & 1.89 & 1.03 & 0.48 & 0.23 \\
\hline
\end{tabular}


Table 18. Bidentate C18 Capacity Factor " $k$ " (MeOH/ABN). Average of in triplicate injections from $10 \%$ to $90 \%$ organic.

\begin{tabular}{|c|c|c|c|c|c|c|c|c|c|c|}
\hline & \multicolumn{10}{|c|}{ Bidentate C18 } \\
\hline & \multirow{2}{*}{ Acidic/Basic/Neutral } & \multicolumn{9}{|c|}{ Mobile Phase Composition (\% Methanol) } \\
\hline & & 10 & 20 & 30 & 40 & 50 & 60 & 70 & 80 & 90 \\
\hline \multirow{7}{*}{ 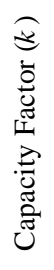 } & Ketoprofen & & & & & 17.2 & 4.72 & 1.68 & 0.58 & 0.3 \\
\hline & 2-Nitrobenzoic Acid & 8.99 & 5.01 & 2.84 & 1.58 & 0.86 & 0.46 & 0.27 & 0.14 & 0.08 \\
\hline & Amitriptyline & & & & & 13.14 & 3.7 & 1.41 & 0.65 & 0.45 \\
\hline & Diphenhydramine & & & & & 3.82 & 1.29 & 0.57 & 0.32 & 0.24 \\
\hline & Anisole & & & & 24.01 & 11.4 & 5.15 & 2.58 & 1.16 & 0.72 \\
\hline & Naphthalene & & & & & & 18.95 & 7.58 & 2.62 & 1.4 \\
\hline & Ethylbenzene & & & & 13.44 & 6.09 & 2.73 & 1.46 & 0.71 & 0.48 \\
\hline
\end{tabular}

Table 19. Phenyl Hydride Capacity Factor " $k$ " (MeOH/ABN). Average of in triplicate injections from $10 \%$ to $90 \%$ organic.

\begin{tabular}{|c|c|c|c|c|c|c|c|c|c|c|}
\hline & \multicolumn{10}{|c|}{ Phenyl Hydride } \\
\hline & \multirow{2}{*}{ Acidic/Basic/Neutral } & \multicolumn{9}{|c|}{ Mobile Phase Composition (\% Methanol) } \\
\hline & & 10 & 20 & 30 & 40 & 50 & 60 & 70 & 80 & 90 \\
\hline \multirow{7}{*}{ 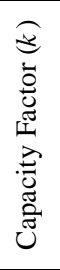 } & Ketoprofen & & & & 18.19 & 4.79 & 0.66 & -0.17 & -0.53 & -0.75 \\
\hline & 2-Nitrobenzoic Acid & 3.7 & 2.47 & 1.63 & 0.85 & 0.31 & -0.14 & -0.3 & -0.5 & -0.7 \\
\hline & Amitriptyline & & & 17.29 & 3.65 & 0.59 & -0.38 & -0.6 & -0.73 & -0.84 \\
\hline & Diphenhydramine & & 13.51 & 3.58 & 0.71 & -0.15 & -0.52 & -0.65 & -0.74 & -0.85 \\
\hline & Anisole & 13.08 & 7.91 & 4.53 & 2.19 & 0.83 & -0.03 & -0.36 & -0.58 & -0.76 \\
\hline & Naphthalene & & & 23.88 & 9.26 & 3.31 & 0.61 & -0.15 & -0.51 & -0.75 \\
\hline & Ethylbenzene & 20.83 & 9.84 & 4.75 & 2.03 & 0.73 & -0.06 & -0.36 & -0.57 & -0.76 \\
\hline
\end{tabular}

Table 20. Diol Capacity Factor " $k$ " (MeOH/ABN). Average of in triplicate injections from $10 \%$ to $90 \%$ organic.

\begin{tabular}{|c|c|c|c|c|c|c|c|c|c|c|}
\hline & \multicolumn{10}{|c|}{ Diol } \\
\hline & \multirow{2}{*}{ Acidic/Basic/Neutral } & \multicolumn{9}{|c|}{ Mobile Phase Composition (\% Methanol) } \\
\hline & & 10 & 20 & 30 & 40 & 50 & 60 & 70 & 80 & 90 \\
\hline \multirow{7}{*}{ 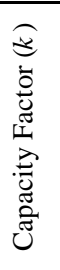 } & Ketoprofen & 7.09 & 1.7 & 0.54 & 0.18 & 0.05 & -0.03 & -0.05 & -0.13 & -0.23 \\
\hline & 2-Nitrobenzoic Acid & 0.24 & 0.16 & 0.12 & 0.06 & 0.04 & 0 & -0.03 & -0.08 & -0.18 \\
\hline & Amitriptyline & 5.6 & 1.62 & 0.64 & 0.25 & 0.09 & 0 & -0.04 & -0.12 & -0.24 \\
\hline & Diphenhydramine & 2.16 & 0.72 & 0.33 & 0.12 & 0.06 & -0.01 & -0.04 & -0.12 & -0.23 \\
\hline & Anisole & 0.73 & 0.41 & 0.25 & 0.13 & 0.05 & -0.01 & -0.04 & -0.1 & -0.21 \\
\hline & Naphthalene & 5.24 & 2.18 & 1.01 & 0.43 & 0.18 & 0.04 & -0.01 & -0.1 & -0.22 \\
\hline & Ethylbenzene & 2.23 & 0.79 & 0.37 & 0.17 & 0.08 & 0.01 & -0.03 & -0.1 & -0.21 \\
\hline
\end{tabular}




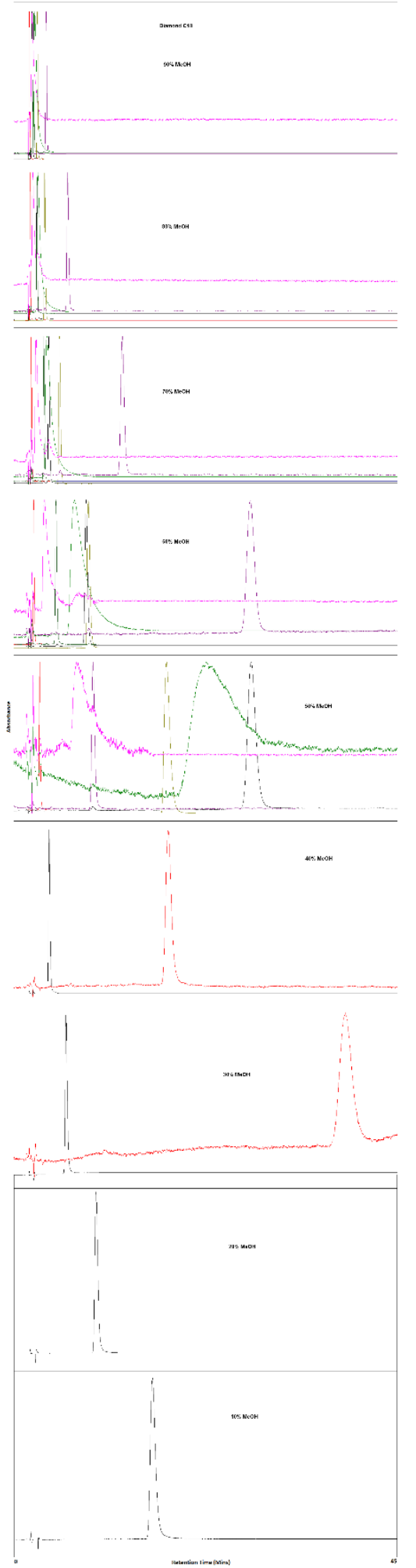

Figure 35. Diamond Bidentate C18 with Methanol Mobile Phase Overlaid/Stacked Chromatograms. 


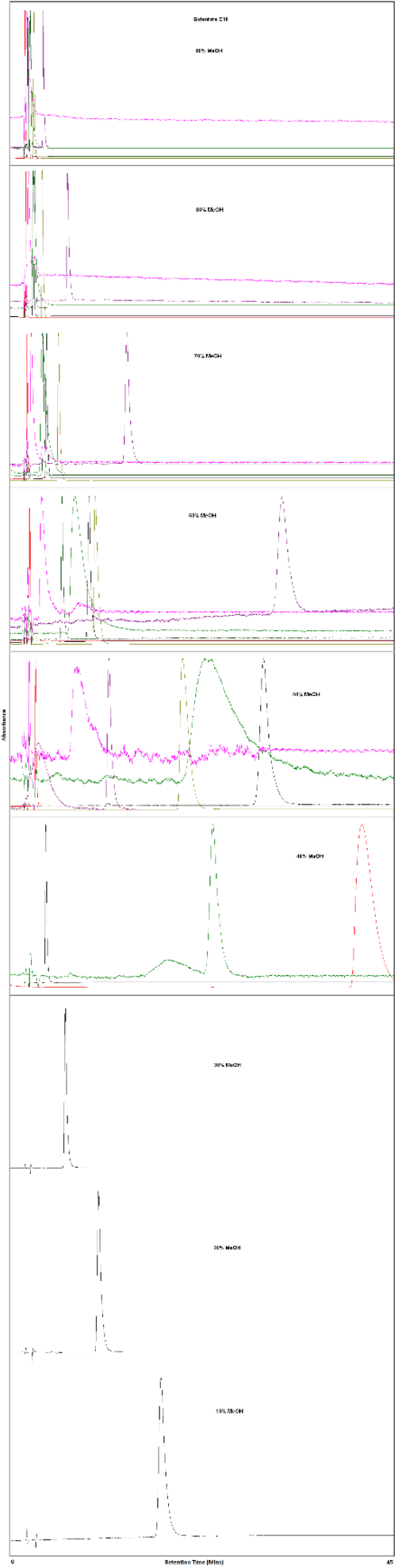

Figure 36. Bidentate C18 with Methanol Mobile Phase Overlaid/Stacked Chromatograms. 
In comparison, the Phenyl Hydride was capable of achieving high resolution without suffering from significant band broadening (Figure 37). Unfortunately, the Diol still does not offer much in the areas of retention even when used with methanol (Figure 38). The only notable difference between retention trends of these compounds was the Phenyl Hydride displaying the expected retention order for Trend 3 (Naphthalene > Ethylbenzene > Anisole) compared to acetonitrile supporting the retention of anisole over ethylbenzene. The most interesting difference between the use of each mobile phase is that the ANP properties are only observed when acetonitrile is used and not at all with methanol (Figures 39 - 42). Although the reason for this result is uncertain, the fact that acetonitrile is a stronger organic may assist in the competition between ANP retention and RP retention. 


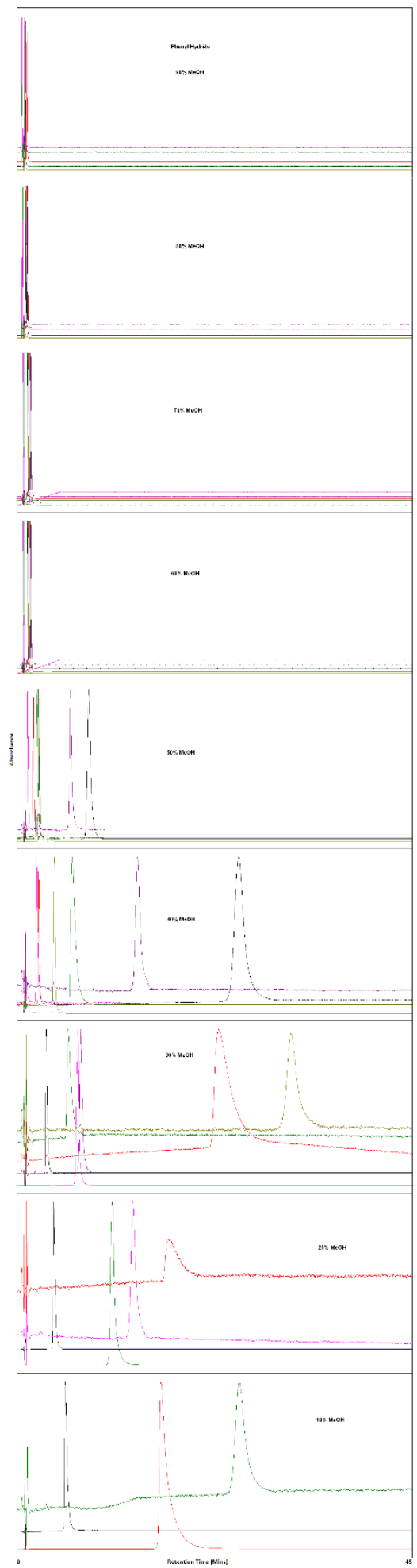

Figure 37. Phenyl Hydride with Methanol Mobile Phase Overlaid/Stacked Chromatograms. 


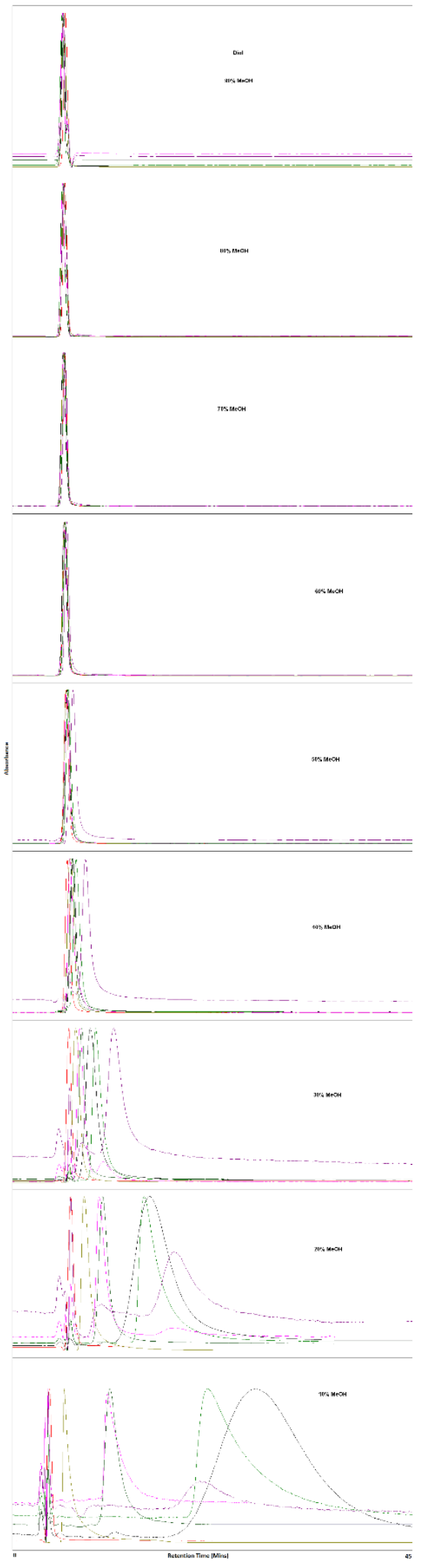

Figure 38. Diol with Methanol Mobile Phase Overlaid/Stacked Chromatogram. 


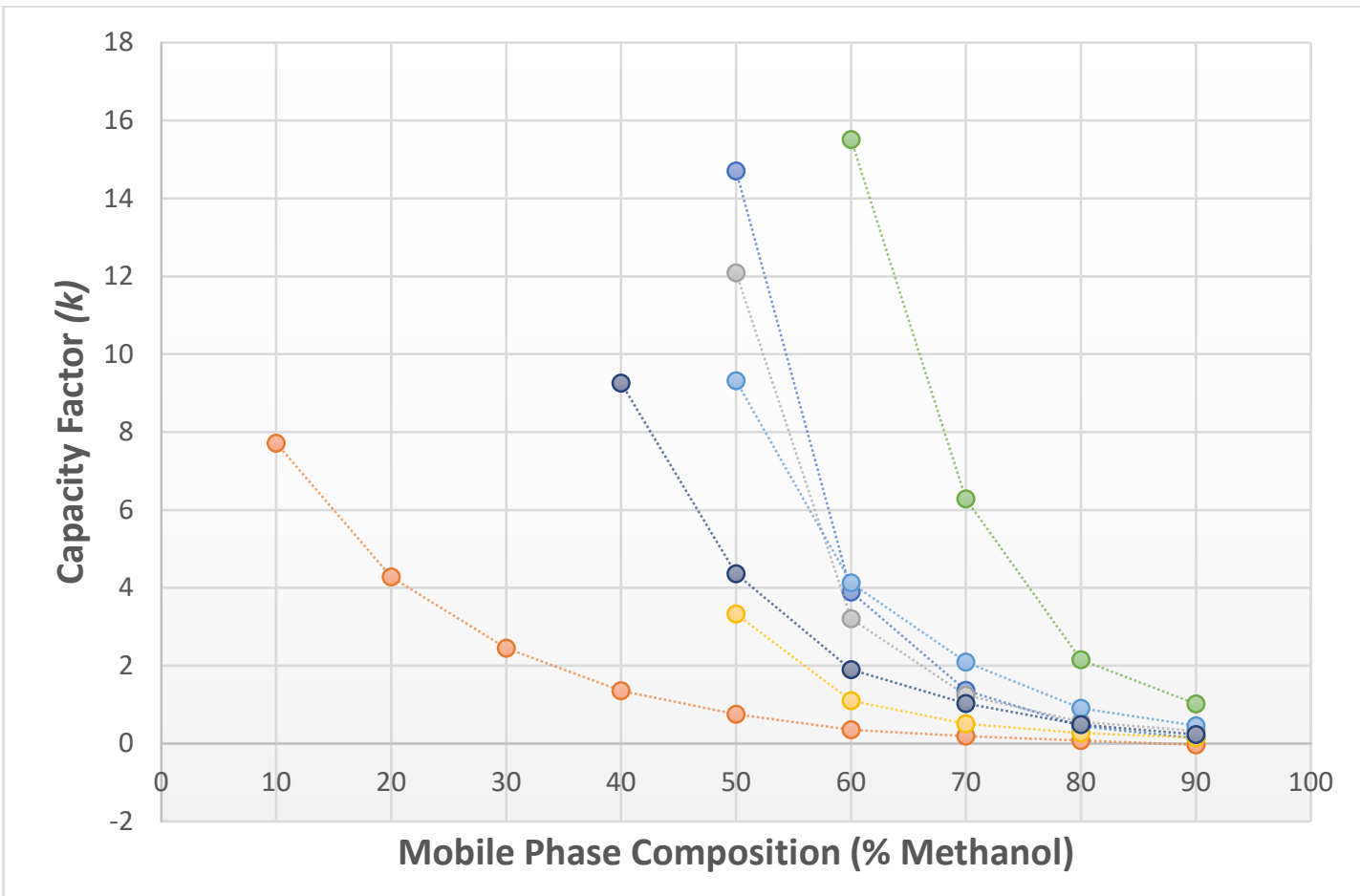

Figure 39. Diamond C18 Retention Map (MeOH/ABN). Compares capacity factor " $k$ " (y-axis) to percentage mobile phase (x-axis).

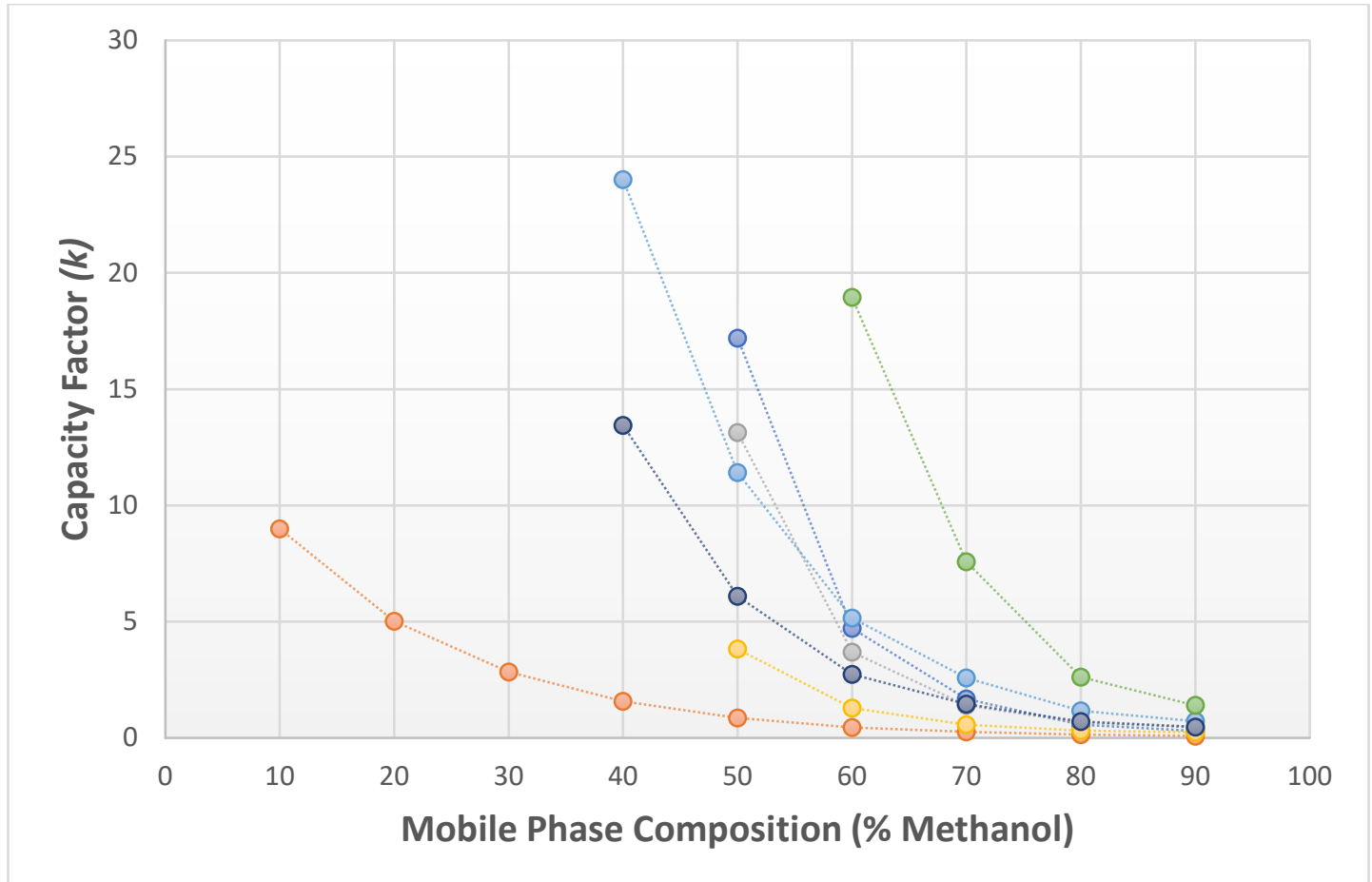

Figure 40. Bidentate C18 Retention Map (MeOH/ABN). Compares capacity factor " $k$ " (y-axis) to percentage mobile phase (x-axis). 


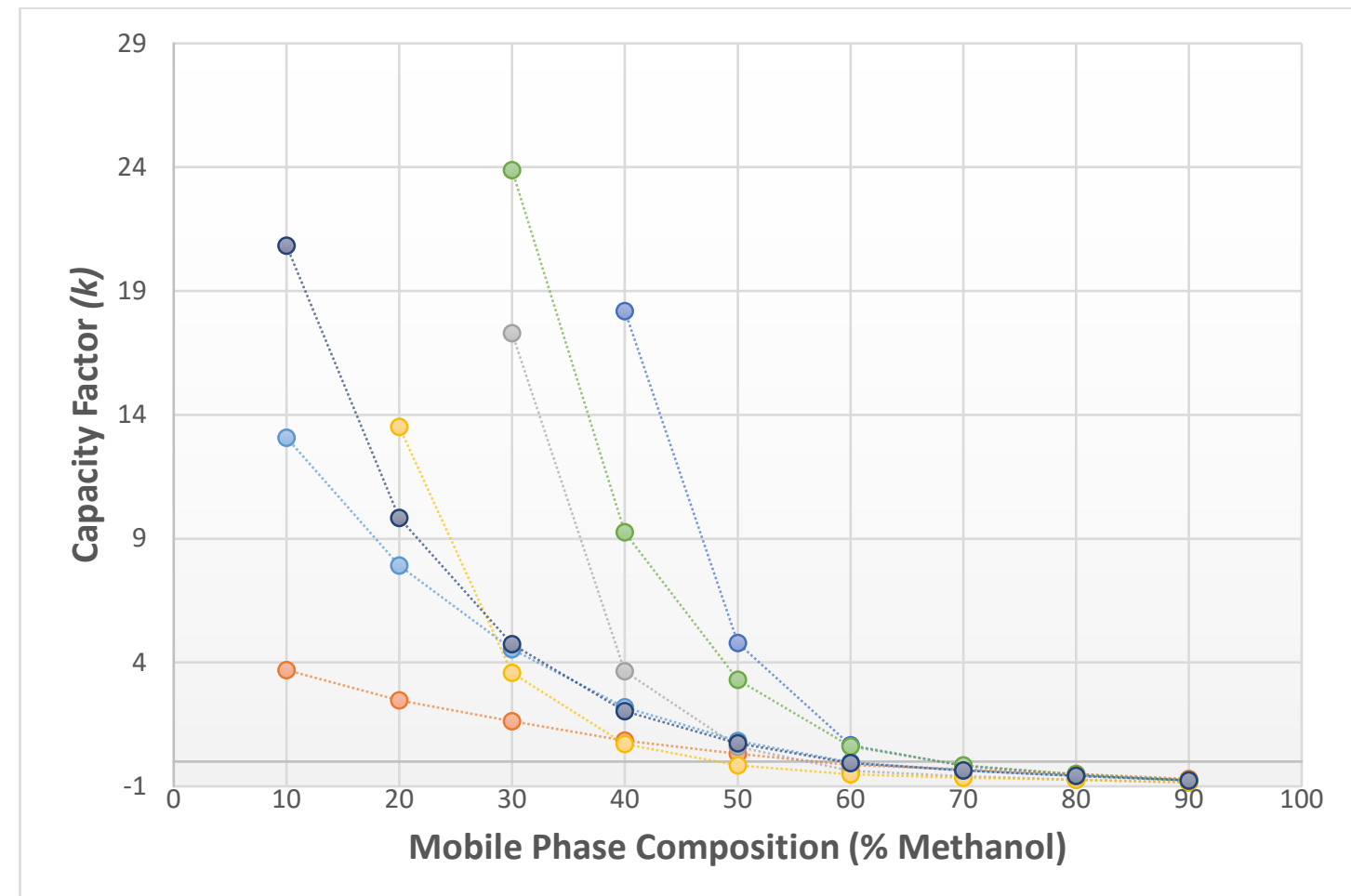

Figure 41. Phenyl Hydride Retention Map (MeOH/ABN). Compares capacity factor “ $k$ ” (y-axis) to percentage mobile phase (x-axis).

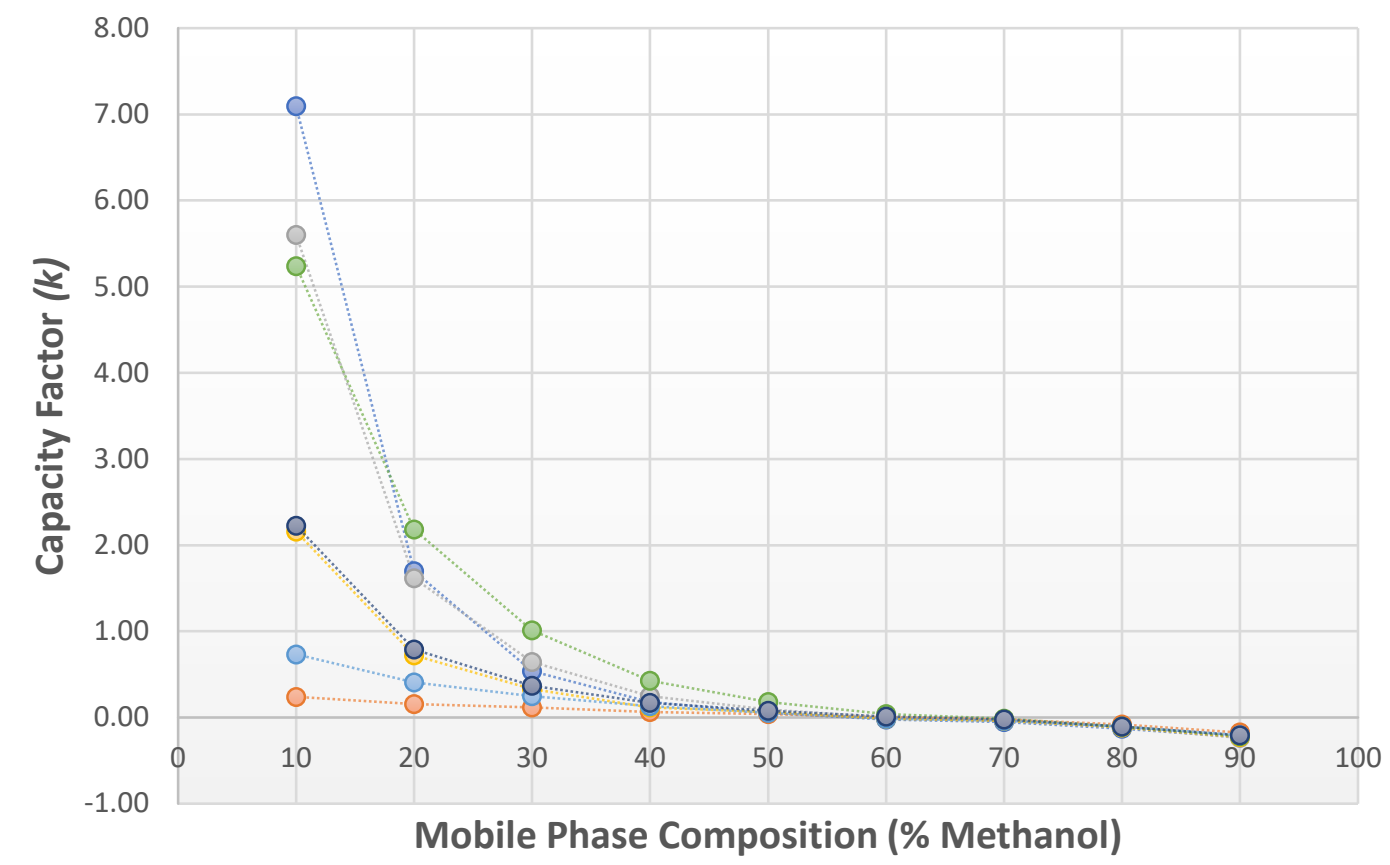


Figure 42. Diol Retention Map (MeOH/ABN). Compares capacity factor " $k$ ” (y-axis) to percentage mobile phase (x-axis).

\section{Chapter 4: Conclusions and Future Studies}

The main purpose for this project was to characterize the four Type-C silica stationary phases by gathering retention data of resveratrol analogues and a small set of acidic, basic and neutral compounds. Retention maps were created by comparing the retention factor $k$ and mobile phase compositions ranging from 90\% organic (acetonitrile vs. methanol) to $10 \%$ organic. Retention trends were organized based on the compound structure and polarity (determined by calculated $\log P$ values). Finally, the overlaid and stacked chromatograms were prepared to demonstrate each column’s theoretical ability to resolve each peak if the compounds were introduced as a mixture. The information collected gave valuable insight regarding the retention power, selectivity and resolution of each column when specific mobile phase was used to analyze the two sets of compounds.

To summarize, there were several key pieces of data that proved informative and interesting. First, higher retention was observed using methanol compared to acetonitrile which was expected due to methanol being a weaker organic. Between the stationary phases the highest retention observed with acetonitrile was as follows: Diamond C18 > Bidentate C18 > Phenyl Hydride > Diol. Compared to methanol, the order changed to: Bidentate C18 > Diamond C18 > Phenyl Hydride > Diol. Secondly, the actual retention trends showed selectivity for compounds apart from polarity. For example, the amide resveratrol retained more than the amine/imine versions at higher aqueous mobile phase compositions. Additionally, surfactant like properties were observed for compounds with 
hydrophilic heads and hydrophobic tails such as MH4 > MH5 (triol/diol), MH7 > MH8 (unsaturated diols) and MH15 > MH16 (saturated diols). While selectivity did vary between the four stationary phases and two mobile phases, the column that demonstrated the most selectivity Phenyl Hydride. The Phenyl showed the highest selectivity for the meta/para hydroxides (MH17 and MH18), unsaturated vs. saturated resveratrol and gave high resolution for both compound sets at low organic content. Aside from methanol demonstrating higher retention of the analytes compared to acetonitrile, methanol did not appear to support ANP retention of MH2 (imine resveratrol), amitriptyline and diphenhydramine. This is important to note that the use of a stronger organic mobile phase may be necessary to promote ANP retention. Another interesting factor was that only nitrogenous compounds where the nitrogen atom was only bound to carbon demonstrated ANP properties. This is most evident when comparing imine, amine and amide versions of resveratrol on the Diol because only the imine version shows ANP retention. All the capacity factor data collected has been sent to collaborators from Monash University for LSER analysis and zeta potential measurements.

Despite all the data gathered for this project future work can still be pursued. Other forms of characterizing the columns may include $\mathrm{pH}$ studies where all other conditions such as mobile phase composition, flow rate and injection volume remain constant while varying the $\mathrm{pH}$ of the solvent (within the recommended limits of each column). Controlling the $\mathrm{pH}$ may shed light on any ionic interactions that may occur depending on whether the analytes are suppressed or not. Similarly, temperature studies can be observed in the same fashion where the temperature of the column is controlled. 
Temperature studies plays a role in the viscosity of solvent, kinetic and thermodynamics which can change retention properties of the stationary phase. Other potential studies may focus on the solvation effects of the mobile phase on the analytes. Since the resveratrol compounds are polyphenols, the hydroxide groups may be susceptible to being solvated by acetonitrile or methanol. One interesting thought provided by Drs. Chester Simocko and Madalyn Radlauer was to derivatize the resveratrol compounds with methoxymethyl acetal groups and compare the retention when methanol is used as mobile phase. The idea is to mimic the proposed "solvation" effect when methanol is used as mobile phase. If the derivatives show the same retention times on the RP columns then the solvation effect may be responsible for some of the differences in retention order mentioned previously. 


\section{References}

1. Cassidy, H. G. The nature of chromatography. J. Chem. Educ. 1956, 33, 482.

2. Heines, S. V. Three who pioneered in chromatography. J. Chem. Educ. 1969, 46, 315.

3. Williams, K. R. Colored Bands: History of Chromatography. J. Chem. Educ. 2002, 79, 922.

4. Robinson, T. Michael Tswett. J. Chem. Educ. 1959, 36, 144.

5. Lee, M. L.; Bowerbank, C. R. Universal Chromatography for Fast Separations. In Unified ChromatographyAmerican Chemical Society: 1999; Vol. 748, pp 179-202.

6. Serban C. Moldoveanu, Victor David Essentials in Modern HPLC Separations; Elsevier: Waltham, MA, 2013; .

7. Ilbeigi, V.; Tabrizchi, M. Thin Layer Chromatography-Ion Mobility Spectrometry (TLC-IMS). Anal. Chem. 2015, 87, 464-469.

8. Costanzo, S. J. High performance thin layer chromatography. J. Chem. Educ. 1984, 61, 1015.

9. Pesek, J.; Matyska, M. T. A Comparison of Two Separation Modes: HILIC and Aqueous Normal Phase Chromatography. LCGC North America 2007, 25, 480.

10. Martin, J. P.; Synge, R. L. M. A New Form of Chromatogram Employing Two Liquid Phases. Biochem. J. 1941, 35.

11. Snyder, L. R. Modern Practice of Liquid Chromatography: Before and after 1971. J. Chem. Educ. 1997, 74, 37.

12. De Vos, J.; Broeckhoven, K.; Eeltink, S. Advances in Ultrahigh-Pressure Liquid Chromatography Technology and System Design. Anal. Chem. 2016, 88, 262-278.

13. Valent Ã£o, P.; andrade, P. B.; Areias, F.; Ferreres, F.; Seabra, R. M. Analysis of Vervain Flavonoids by HPLC/Diode Array Detector Method. Its Application to Quality Control. J. Agric. Food Chem. 1999, 47, 4579-4582.

14. Pesek, J. J.; Matyska, M. T. Amazon Bark Extract. 2015, 331.

15. Brettell, T. A.; Butler, J. M.; Almirall, J. R. Forensic Science. Anal. Chem. 2007, 79, 4365-4384. 
16. Pesek, J. J.; Matyska, M. T. Codeine and Hydrocodone. 2014, 315.

17. McGorrin, R. J. One Hundred Years of Progress in Food Analysis. J. Agric. Food Chem. 2009, 57, 8076-8088.

18. Pesek, J. J.; Matyska, M. T. Oleuropein in Olive Leaves. 2013, 283.

19. Pesek, J. J.; Matyska, M. T. Analysis of Harmful Substances in Dietary Supplements. 2015, 337.

20. Qu, G.; Shi, J.; Wang, T.; Fu, J.; Li, Z.; Wang, P.; Ruan, T.; Jiang, G. Identification of Tetrabromobisphenol A Diallyl Ether as an Emerging Neurotoxicant in Environmental Samples by Bioassay-Directed Fractionation and HPLC-APCIMS/MS. Environ. Sci. Technol. 2011, 45, 5009-5016.

21. Del Valle, P. L.; Muldoon, M. T.; Karns, J. S.; Nelson, J. O.; Mulbry, W. W. Development of Improved Immunoassay and HPLC Methods for the Analysis of Chlorodiamino-s-triazine in Environmental Samples. J. Agric. Food Chem. 1996, 44, 945-952.

22. Pesek, J. J.; Matyska, M. T. Analysis of Herbicides. 2014.

23. Moldoveanu, S. C.; David, V. Essentials In Modern HPLC Separations; Elsevier: MA, USA, 2013; .

24. Qiu, H.; Liang, X.; Sun, M.; Jiang, S. Development of silica-based stationary phases for high-performance liquid chromatography. Analytical and Bioanalytical Chemistry 2011, 399, 3307-3322.

25. Wyndham, K. D.; O'Gara, J. E.; Walter, T. H.; Glose, K. H.; Lawrence, N. L.; Alden, B. A.; Izzo, G. S.; Hudalla, C. J.; Iraneta, P. C. Characterization and Evaluation of C18 HPLC Stationary Phases Based on Ethyl-Bridged Hybrid Organic/Inorganic Particles. Anal. Chem. 2003, 75, 6781-6788.

26. Brown, L.; Ciccone, B.; Pesek, J. J.; Matyska, M. T. An Evolution in Separation Media for HPLC. American Laboratory 2003, 23.

27. Pesek, J. J.; Matyska, M. T.; Oliva, M.; Evanchic, M. Synthesis and characterization of bonded phases made via hydrosilation of alkynes on silica hydride surfaces. Elsevier 1998, 818, 145.

28. Pace-Asciak, C. R.; Hahn, S.; Diamandis, E. P.; Soleas, G.; Goldberg, D. M. The red wine phenolics trans-resveratrol and quercetin block human platelet aggregation and 
eicosanoid synthesis: implications for protection against coronary heart disease. Clincal Chimica Acta 1995, 235, 207.

29. Frankel, E. N.; Waterhouse, A. L.; Kinsella, J. E. Inhibition of human LDL oxidation by resveratrol. Lancet 1993, 341, 1103.

30. Jang, M.; Cai, L.; Udeani, G. O.; Slowing, K. V.; Thomas, C. F.; Beecher, C. W. C. W.; et al. Cancer chemopreventive activity of resveratrol, a natural product derived from grapes. Science 1997, 275, 218.

31. Zhou, J.; Zhou, M.; Yang, F.; Liu, C.; Pan, R.; Chang, Q.; Liu, X.; Liao, Y. Involvement of the Inhibition of Intestinal Glucuronidation in Enhancing the Oral Bioavailability of Resveratrol by Labrasol Containing Nanoemulsions. Mol. Pharmaceutics 2015, 12, 1084-1095.

32. Azzolini, M.; Mattarei, A.; La Spina, M.; Marotta, E.; Zoratti, M.; Paradisi, C.; Biasutto, L. Synthesis and Evaluation as Prodrugs of Hydrophilic Carbamate Ester Analogues of Resveratrol. Mol. Pharmaceutics 2015, 12, 3441-3454.

33. Szekeres, T.; Fritzer-Szekeres, M.; Saiko, P.; Jager, W. Resveratrol and Resveratrol Analogues-Structure-Activity Relationship. Pharmaceutical Research 2010, 27, 1042.

34. Letter, W. Any advatages of Agilent G1379 1260 Micro Degasser towards 'normal' G1322 1200 or G1322A 1100 Degasser for analytical HPLC?? 2017.

35. Sanchez, C. A. agilent 1200 vs. 1100 binary pumps. 2011.

36. Singh, G.; Pai, R. S.; and Pandit, V. Development and validation of a HPLC method for the determination of trans-resveratrol in spiked human plasma. Journal of Advanced Pharmaceutical Technology and Research 2012, 3, 130.

37. Sadek, P. C.; Carr, P. W.; Doherty, R. M.; Kamlet, M. J.; Taft, R. W.; Abraham, M. $\mathrm{H}$. Study of retention processes in reversed-phase high-performance liquid chromatography by the use of the solvatochromic comparison method. Anal. Chem. 1985, 57, 2971-2978.

38. Kulsing, C.; Yang, Y.; Sepehrifar, R.; Lim, M.; Toppete, J.; Matyska, M. T.; Pesek, J. J.; Boysen, R. I.; Hearn, M. T. W. Investigations into the separation behaviour of perfluorinated C8 and undecanoic acid modified silica hydride stationary phases. Anal. Chim. Acta 2016, 916, 102-111. 
39. Wilson, N. S.; Nelson, M. D.; Dolan, J. W.; Snyder, L. R.; Wolcott, R. G.; Carr, P. W. Column selectivity in reversed-phase liquid chromatography: I. A generalquantitative relationship. Journal of Chromatography 2002, 961, 171. 